\title{
Art-Directed Composition in Dynamic Real Scenes
}

by

\author{
Lohit Petikam
}

\begin{abstract}
A thesis submitted to the
Victoria University of Wellington

in fulfilment of the requirements for the degree of

Doctor of Philosophy

in Faculty of Computer Science and Engineering
\end{abstract}

Victoria University of Wellington

2021 



\section{Supervisory Committee}

Primary Supervisor: Associate Professor Taehyun Rhee

Secondary Supervisor: Professor Ken Anjyo

School of Engineering and Computer Science,

Victoria University of Wellington 


\section{Abstract}

Art direction is crucial for films and games to maintain a cohesive visual style. This involves carefully controlling visual elements like lighting and colour to unify the director's vision of a story. With today's computer graphics (CG) technology 3D animated films and games have become increasingly photorealistic. Unfortunately, art direction using CG tools remains laborious. Since realistic lighting can go against artistic intentions, art direction is almost impossible to preserve in real-time and interactive applications. New live applications like augmented and mixed reality (AR and MR) now demand automatically art-directed compositing in unpredictably changing real-world lighting.

This thesis addresses the problem of dynamically art-directed 3D composition into real scenes. Realism is a basic component of art direction, so we begin by optimising scene geometry capture in realistic composites. We find low perceptual thresholds to retain perceived seamlessness with respect to optimised real-scene fidelity. We then propose new techniques for automatically preserving art-directed appearance and shading for virtual 3D characters. Our methods allow artists to specify their intended appearance for different lighting conditions. Unlike with previous work, artists can direct and animate stylistic edits to automatically adapt to changing real-world environments. We achieve this with a new framework for look development and art direction using a novel latent space of varied lighting conditions. For more dynamic stylised lighting, we also propose a new framework for art-directing stylised shadows using novel parametric shadow editing primitives. This is a first approach that preserves art direction and stylisation under varied lighting in AR/MR. 


\section{Acknowledgments}

Thanks to the following people and others who have supported, supervised, advised, tutored, collaborated with, and inspired me throughout my study and career: Andrew Chalmers, Ben Allen, Stephen Thompson, Kieran Carnegie, Joshua Scott, Ping Liu, Ian Loh, Richard Roberts, John Lewis, Siyun Thompson, Mohsin Ali, Warren Butcher, Matt Hicks, Junhong Zhao, Rafael Kuffner dos Anjos, Joaquim Jorge, Daniel Medeiros, Raqi Syed, Areito Echevarria, Jim Foley, Christian Suppan, Simon Finnie, Betty Kim, Rongsen Chen, Lukas Stoecklein, Charlie Hewitt, Diana Siwiak, Neil Dodgson, Fang-Lue Zhang, Todd Zickler, Jacob Grossman, Hiroyuki Ochiai, Jen Gillespie, Peter Hillman, Brian Sharpe, Eugene d'Eon, Pravin Bhat, Robert Higgs.

Special thanks are extended to the staff at OLM Digital whose support, insight, and wisdom made this research possible: Ayumi Kimura, Akinobu Maejima, Anadaroop Mukherjee, Tatsuo Yotsukura, Kazuki Kumagai, Marc Salvati.

My sincere gratitude goes to my academic supervisors Associate Professor Taehyun Rhee and Professor Ken Anjyo who spent countless extra hours developing and strengthening my ideas and research, further than I could achieve alone.

This thesis was also supported by the Entrepreneurial Universities Programme, funded by the Tertiary Education Commission (TEC), New Zealand.

Finally, I thank all my family and friends for their endless support and encouragement throughout my study. 


\section{Publications}

- (Conditionally accepted with minor revisions in Transactions on Graphics)

L. Petikam, T. Rhee, K. Anjyo. "Shading Rig: Dynamic Art-Directable Stylised Shading for 3D Characters"

- (Submitted to Pacific Graphics 2020)

L. Petikam, A. Chalmers, T. Rhee. "Art-directing Appearance using an Environment Map Latent Space"

- L. Petikam, A. Chalmers, and T. Rhee, "Visual Perception of Real World Depth Map Resolution for Mixed Reality Rendering", Proceedings of IEEE Virtual Reality, Reutlingen, Germany, March 2018.

- T. Rhee, L. Petikam, B. Allen, and A. Chalmers, "MR360: Mixed Reality Rendering for 360-degree Panoramic Videos", in IEEE Transactions on Visualization and Computer Graphics, vol. 23, no. 4, pp. 1379-1388, April 2017, presented at IEEE Virtual Reality 2017 and SIGGRAPH 2017. 


\section{Contents}

Supervisory Committee $\quad$ i

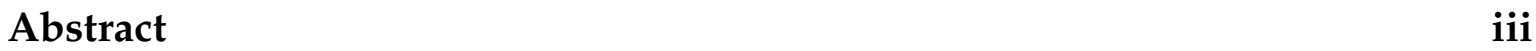

$\begin{array}{ll}\text { Acknowledgments } & \text { v }\end{array}$

Publications vii

List of Figures $\quad$ xiii

List of Tables $\quad$ xxi

1 Introduction 1

1.1 Realistic Compositing ...................... 3

1.2 Art-directed Compositing ..................... 3

1.3 Problem Statement . . . . . . . . . . . . . . . . . . 5

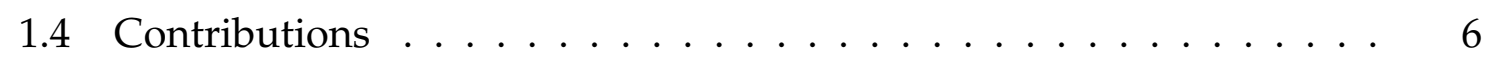

1.5 Thesis Structure $\ldots \ldots \ldots \ldots \ldots \ldots$

2 Visual Perception of Real-World Depth Map Resolution for Mixed Reality Composition 


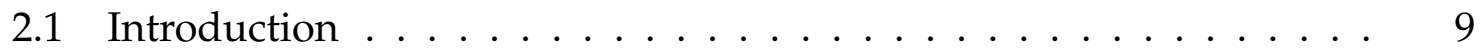

2.2 Related Work . . . . . . . . . . . . . . . . . 12

2.2.1 MR compositing with Depth . . . . . . . . . . . . . 12

2.2.2 Perceptual Studies with Depth . . . . . . . . . . . . 13

2.2.3 Perceptually Based Rendering . . . . . . . . . . . . . 14

2.2.4 Perceptual Studies in Composition . . . . . . . . . . . . . . 14

2.3 Composition with Depth . . . . . . . . . . . . . . 15

2.3.1 Differential Rendering . . . . . . . . . . . . . . . 15

2.3.2 Local Scene Generation from 360 Depth Maps . . . . . . . . . 16

2.3.3 Depth Composition Artifacts . . . . . . . . . . . . . . 18

2.4 User Experiment . . . . . . . . . . . . . . . . . . . 19

2.4 .1 Stimuli ............................ 21

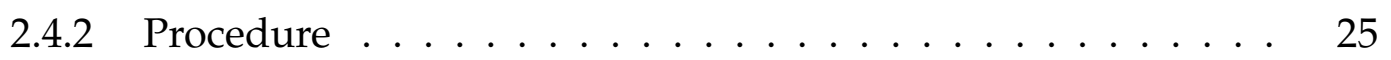

2.5 Results and Analysis . . . . . . . . . . . . . . 26

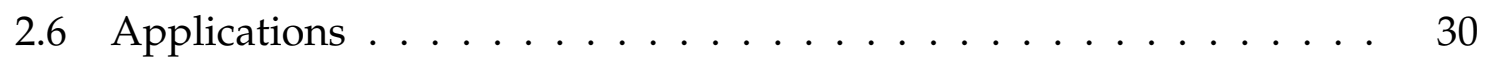

2.7 Conclusion and Future Work . . . . . . . . . . . . . . . . 32

3 Art-directing Appearance using an Environment Map Latent Space 35

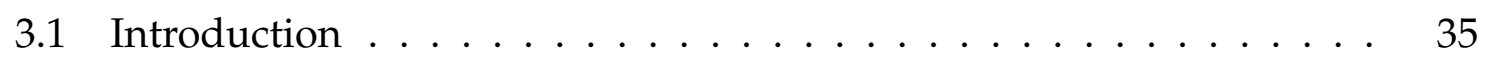

3.2 Related Work . . . . . . . . . . . . . . . . 38

3.3 Method ............................. 40

3.3.1 Data Preparation .................. 40

3.3.2 Learning the Latent Space . . . . . . . . . . . . . . . . . 41

3.3.3 Art Directing Appearance Using the Latent Space . . . . . . . 43

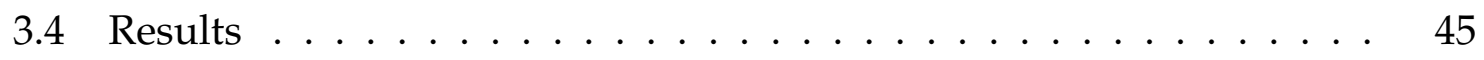


3.4.1 Observed Semantic Clustering . . . . . . . . . . . . . 45

3.4.2 Evaluation ....................... 50

3.4.3 Art direction Application . . . . . . . . . . . 56

3.5 Limitations and Future Work . . . . . . . . . . . . . . . . . . 60

4 Shading Rig: Dynamic Art-Directable Stylised Shading for 3D Characters 63

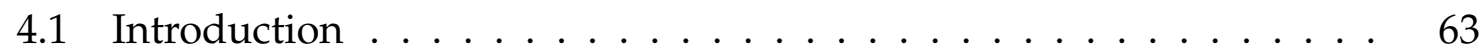

4.2 Related Work . . . . . . . . . . . . . . . . 65

4.2.1 Toon shade editing . . . . . . . . . . . . 65

4.2.2 Artist-Defined Lighting and Stylised Shading _ . . . . . . . 67

4.2.3 2D Shape Interpolation $\ldots \ldots$. . . . . . . . . . 68

4.2.4 Summary of Prior Work and Comparison . . . . . . . . . . 69

4.3 Shading Rig Overview . . . . . . . . . . . . . . . 70

4.3.1 Shade Editing Workflow . . . . . . . . . . . . . 73

4.3.2 Animation Workflow . . . . . . . . . . . . . . 73

4.3.3 Shading Edit Parameters . . . . . . . . . . . . . . 74

4.3.4 Edit Blending and Deformation . . . . . . . . . . . 75

4.3.5 Local Editing Control . . . . . . . . . . . . 76

4.4 Shading Rig Edit Model . . . . . . . . . . . . . . 77

4.4.1 Intensity Distribution $\ldots \ldots \ldots 78$

4.4.2 Shading Rig Animation . . . . . . . . . . . . . 83

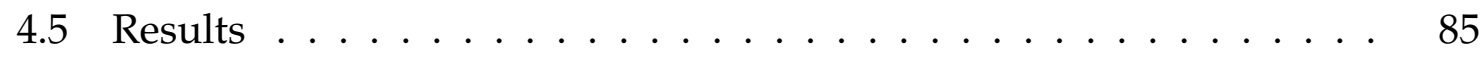

4.5.1 Examples and Use-Cases . . . . . . . . . . . . . 85

4.5.2 Shadow animation with lighting changes . . . . . . . . . 9 90

4.5.3 Comparison With Previous Work . . . . . . . . . . . . . . . 92 
4.5 .4 Artist Feedback . . . . . . . . . . . . . . . . . 97

4.6 Limitations and Future Work . . . . . . . . . . . . . . . . 101

$\begin{array}{lll}5 & \text { Conclusion } & 103\end{array}$

5.1 Achieved Goals . . . . . . . . . . . . . . . . . . . 103

5.2 Limitations . . . . . . . . . . . . . . . . . . . . 104

5.3 Future Work . . . . . . . . . . . . . . . . . 105

$\begin{array}{ll}\text { A Shading Rig Appendix } & 107\end{array}$

A.1 Local Texture Space . . . . . . . . . . . . . . . . . . . . . 107

A.2 Symbols and Notation . . . . . . . . . . . . . . . . . 108

A.3 Shading Interpolation Result _ . . . . . . . . . . . . . . . 109

A.4 Performance Evaluation . . . . . . . . . . . . . . . . . 109

A.5 Artist Feedback Prompts . . . . . . . . . . . . . . . . . . . . 110

$\begin{array}{ll}\text { Ethics } & 117\end{array}$

$\begin{array}{ll}\text { Bibliography } & 121\end{array}$ 


\section{List of Figures}

2.1 Examples of compositing foreground objects (statue, teapot and armadillo) into the background (Bedroom scene) using its depth map. Comparison between ground truth reference rendering with high resolution 3D meshes (a), composition with high resolution depth map $(4096 \times 2048)(b)$, composition with perceptually optimal depth resolution $(426 \times 213)(\mathrm{c})$, and composition with insufficient depth resolution $(64 \times 32)$ (d) showing noticeable artifacts from poorly reconstructed geometry. . . . . . . . . . . . . .

2.2 Compositing steps for differential rendering. (a) Background image, (b) Reconstructed local scene mesh from depth map, (c) Local scene rendered with virtual object, (d) Object matte, (e) Change in local scene: (c) divided by (b) excluding object, (f) Final Composite with object and its shadow. . . . . . . . . . . . . . .

2.3 Artifact types and their behaviour as depth map resolution decreases. The bottom labels indicate the depth resolution used in each column. The top row (LS) shows the Type 1 scene's geometry reconstruction as an example. The decreasing depth map resolution causes the geometry to degrade, creating the artifacts in Type 1. . . 
2.4 How the Type 4 shadow overlap artifact occurs in differential rendering. The left image and close-up are the background image. Column (a) is the local scene, (b) is the local scene with objects, (c) is the error, and (d) is the final composite. Row (1) shows the process using a $64 \times 32$ resolution depth map, while row (2) uses $4096 \times 2048$. .

2.5 Top row: ground truth renderings of the scenes used in our experiment. Bottom row: example stimuli with the left most object being the inserted object in each scene. Type 2 (c) and Type 4 (d) share the same scene but with different artifacts masked out in the

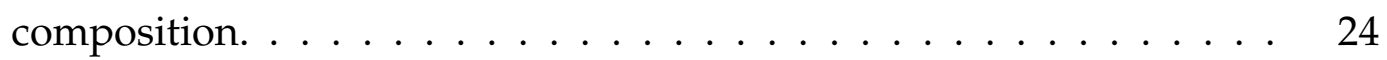

2.6 Experiment results of artifact noticeability across all types and objects, against depth map width (height is half width). Lower noticeability values are better. Objects 1, 2 and 3 are the left, middle and right objects, respectively, in each Type's scene. . . . . . . . . . . . .

2.7 Average artifact noticeability against depth map width, for all types. The dotted line at noticeability $=0.5$ indicates where the perceptual thresholds are determined. The dotted line at noticeability $=1.0$ indicates where artifacts become slightly noticeable. Lower noticeability values are better. . . . . . . . . . . . .

2.8 Example application of the Type 1 perceptual threshold in MR compositing into a photorealistic background. The glossy teapot, statue and white chair, are the inserted objects. Compositions with a $4096 \times 2048$ depth map (a) and a perceptually optimal $426 \times 213$ depth map (b). Reconstructed geometry for the composites is also shown: meshes with $4096 \times 2048$ (c) and $426 \times 213$ (d) depth maps. Top row: composition into a background scene with continuous depth (couch object). Bottom row: fail case of composition into discontinuous depth (car object with artifacts around wheels in both (b) and (c)). . . 
3.1 a) Our environment map (EM) latent space expresses semantic clustering in 2D, for navigating a varied EM database during look development. b) Art directing material and lighting edits across the space is achieved by 2D painting. c) Art-directed appearance is preserved in similar EMs, and dynamically adapted to changing environment lighting. Character model by Emiliano Colantoni, licensed under CC BY 4.0. . . . . . . . . . . . . . . . . .

3.2 Explained variance ratio from PCA dimensions of 3D embedding, for different $w_{S H}$. Between 0.6 and 0.7 , variance is almost evenly shared across two dimensions for our training set. . . . . . . . . . . .

3.3 a) Using only $\mathrm{SH}\left(w_{S H}=0\right)$, indoor EMs (mostly warm colours) and outdoor EMs (mostly cool colours) are embedded too close together. b) Weighting $\mathrm{SH}$ by 0.8 leads to a slight distance increase, but not meaningful clustering. c) Weighting $\mathrm{SH}$ by 0.68 successfully forms a distinct indoor EM cluster, while preserving sunny and overcast clusters. d) If $w_{S H}$ is too high, the embedding is biased toward colour and devalues lighting properties. . . . . . . . . . . . .

3.4 Latent space EMs plotted as points coloured using their mean RGB colour. We observe overcast, sunny, and indoor EM clusters shown by soft, hard, and multiple shadows, respectively, rendered below. Light elevation (seen in highlight positions and shadow length) varies consistently across the clusters in the $x$-axis. . . . . . . . . . . .

3.5 Samples found in the observed outdoor overcast cluster of our latent space. . . . . . . . . . . . . . . . . . .

3.6 Samples in the observed outdoor sunny cluster. . . . . . . . . . .

3.7 Samples in the observed indoor cluster. . . . . . . . . . . .

3.8 Samples between overcast and sunny clusters. From 1-6, we see a transition from heavy cloud, to clear sky with sun occluded, to partial sun occlusion (almost sunny) . . . . . . . . . . . . . . 
3.9 Samples between sunny and indoor clusters. From 1-4, we see a transition from fully outdoor and sunny, to large window lighting, to small window lighting, to artificial lighting. . . . . . . . . . .

3.10 Encoding a test set of unseen EMs into our latent space. The new samples are concentrated in the same clusters observed in the original embedding. . . . . . . . . . . . . . . . .

3.11 Encoding unseen EMs from the HDRI-Haven outdoor dataset [1]. The new samples are concentrated in the same clusters observed in the original embedding. . . . . . . . . . . . . . .

3.12 Application of the latent space as an appearance control space. Top: Lighting environments used in look-dev, with 3D character preview under the same lighting below. In the third preview, highlights are lacking. Bottom: latent space is used to specify increased glossiness, by painting brighter intensity values in the region of cloudy EMs. This enhances highlights only in cloudy scenes which avoids overexposing them in sunny and outdoor scenes. . . . . . . . . .

3.13 Top: EM latent space with rim light intensity values painted such that rim light decreases when transitioning from sunny to sunset EMs (14). Below: comparison between a fixed edited appearance, and with our dynamic art-directed appearance. . . . . . . . . . . . . .

3.14 Highly varied stylised appearance can be art-directed across different lighting environments. Outline thickness and shading softness in cartoon shading is varied independently. . . . . . . . .

3.15 Failure cases: Some artificial-light indoor EMs are embedded in the outdoor-overcast cluster (1-3). One snowy overcast EM was wrongly embedded in the sunny cluster. . . . . . . . . . . . . . 
4.1 Top-left: key light adjusted for base toon shadows to be edited. Top-right: edit placed near surface mesh to adjust shading boundary. Bottom: parameters controlling the shape of the edit (in this case, increasing Anisotropy and Sharpness creates the desired sharp contour). . . . . . . . . . . . . . . . 70

4.2 Top row: Example sequence of shape manipulation for one shading edit, with the parameter being changed below (previous shape shown with dotted lines). Underneath is the same manipulation on a sphere.

4.3 Modes for blending edits together, and tracking surface deformations during animation. . . . . . . . . . . . . . . .

4.4 Steps to animate a single edit with lighting changes. a) Unedited shading from key light. b) Shading rig edit placed to edit shading boundary. c) Key light rotated and shading must be edited again. d) First edit is keyframed to move and change shape under new lighting. The edit's position and shape interpolate between keyframes KF1 and KF2 as the key light direction changes. . . . . . .

4.5 Using multiple light origin points when editing shade across different areas on a character. . . . . . . . . . . . . . . . . . . .

4.6 (a) Original intensity distribution $I\left(u_{w}, v_{w}\right)$ with periodicity artifacts when using a large Bend value. (b) Mask created by $c_{w}\left(\theta_{w}\right)$. (c) Intensity distribution masked by (b) which removes the artifacts. (d) Final shape after thresholding. . . . . . . . . . . . . . .

4.7 Given the surface mesh in (a), smoothed normals reduce toon shadow artifacts, but remove important shape details. Edits are placed to indicate the nose mouth in (b). Reducing the Normal Smooth parameter for the mouth edit selectively added detail which the model alone did not express. . . . . . . . . . . . . . . . 
4.8 In (a), conventional toon shadow is the red shape lacking cheek and nose definition. Our edit shapes, shown in blue, are placed to depict more detail. In (b) we see the shadow shapes (outlined) are smoothly combined into the desired shading. The final shading is shown in (c).

4.9 Top: Example hand-drawn shadows used for facial expression and detail. Bottom: unedited toon shading (left) and edited expression using shading edits (right). This result required 5 edits. Creature concept: CWildboy Studios used with permission. Female artwork by Claudio Grassi licensed under CC BY. Head model by Vinicius Nunes licensed under CC BY. . . . . . . . . . . . . . . .

4.10 Top: hand-drawn stylised muscle shading. Bottom: unedited toon shading (left) and added muscle shading using 10 shading edits (right). Artwork by Brain Graft licensed under CC BY. Model by Julien Kaspar licensed under CC BY. . . . . . . . . . . . . . . . .

4.11 Left: Hand-drawn stylised shading with Rembrandt triangle under the eye. Middle: Edited toon shading with the shading rig, applying a Rembrandt triangle without a nose shadow being cast. A single intensity edit is used to blend with existing key lighting. Right: rounded Rembrandt triangle added also using one mask edit with high Normal Smoothing. Character artwork: CRodu used with

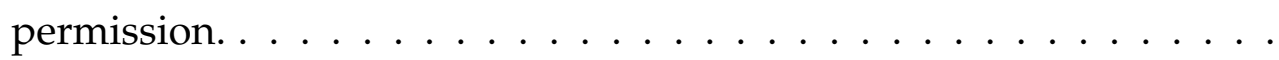

4.12 Left: high detail mesh. Middle: base toon shading. Right: edited result (9 edits). Model by Julien Kaspar licensed under CC BY. . . . . .

4.13 Top: Edit made on the left-most frame (outlined in blue) can track mesh deformation throughout an animation sequence. Bottom: edit shown in white precisely tracks mesh surface parametrisation and remains animatable and mesh/texture resolution independent. Model by Daniel Martinez Lara licensed under CC BY. . . . . . . . .

4.14 Left: Lit-sphere shading [2]. Middle: Edited shading using shading rig (5 edits). Right: Shading Rig (circle and axis controllers) and its resulting intensity field. Model by Julien Kaspar licensed under CC BY. 90 
4.15 Top row (SR): Shadow animation produced by a pre-animated shading rig (collection of cross-shaped locators) as the key light is rotated. Below each large frame: conventional toon shading (TS) and normal smoothed toon shading (NS) to be edited. . . . . . . . . . . .

4.16 Shading rig animation result, used for a dynamic character moving relative to key lighting. TS: toon shading, NS: normal smoothing, SR: shading rig. . . . . . . . . . . . . . . . .

4.17 Example animated shade edit comparison with colour variation from red (start) to blue (end). (a) Offset painting (Todo et al. 2007 [3]) result with 4 keyframes (numbered), showing fragmented motion of shadow. (b) our approach requires only 2 keyframes to yield continuous motion and shape manipulation. . . . . . . . . . .

4.18 Texture mapping using a rasterised shading texture exhibits pixelation artifacts upon magnification (a). Using a rasterised intensity distribution (b) for optimal transport [4] causes artifacts after thresholding (c). Our parametric distribution (d) provides artifact free reproduction of sharp cusps and shape boundary. . . . .

4.19 Shape interpolation running time comparison (log scale) between Instant Transport Maps [4] and our model, using non-parallelised CPU implementations of each method to compare number of operations. . . . . . . . . . . . . . . . . . .

4.20 Original shade edits made on a surface mesh (blue) and their dependence on mesh density. a) Limitation of Todo et al. 2007 [3] where edit shapes are affected by mesh density and topology. b) Our parametric edits preserve their shape regardless of mesh resolution and topology. . . . . . . . . . . . . . . . .

4.21 For the task of compositing cartoons into real-world backgrounds, the shading rig can be applied to environmental lighting. We show toon shading variation with EM rotation, with the shading rig applied (SR) and without (No SR) . . . . . . . . . . . . . . . . . . 
A.1 Limitation of lit-sphere approach [2] for artistic editing. Left: Artist-specified local area on 3D model where shading must be edited. Middle: corresponding area on lit-sphere domain where the stylisation texture would be modified. Right: affected area when applying the edited lit-sphere texture is far greater than the specified region. . . . . . . . . . . . . . . 107

A.2 Light space vectors computed with respect to the light position $\mathrm{p}_{\mathrm{l}}$ and light origin $\mathbf{p}_{\mathbf{0}} \ldots \ldots \ldots \ldots$. . . . . . . . . . . . . . . 109

A.3 Intensity distribution interpolation with the method of [3] (top) and our approach (bottom) on a mesh surface. Todo et al. 2007 [3] linearly blend between fixed distributions, causing shapes to disappear and reappear between two keyframes (left-most and right-most images). Our Shading Rig parametrises the distribution for true shape movement and interpolation. Note that precisely matching the shapes of Todo et al. 2007 [3] with ours is limited by mesh topology. . . . . . . . . . . . . . . . 113

A.4 Time taken to keyframe all parameters for one edit, as its number of keyframes increases, measured by the RBF solve time. . . . . . . . . . 114

A.5 RBF evaluation time (log scale) for shading rigs with 10 and 100 edits, as the number of keyframes per edit increases (all parameters keyframed). . . . . . . . . . . . . . . . . . . . . . 114

A.6 GPU shading frame time (log scale) as the number of edits increases, at different screen percentages of $1920 \times 1080$ resolution. . . . . . . . . 115 


\section{List of Tables}

2.1 Table of depth map resolution perceptual thresholds (PT), slightly noticeable thresholds (SN), and inflection points (IP) from experimental results.

4.1 Previous solutions for dynamic artist-defined shade editing (including 2D shape interpolation). Our approach achieves all requirements simultaneously. . . . . . . . . . . . . 66

4.2 Shading shape model control parameters. . . . . . . . . . . 77

A.1 Symbols and notation. . . . . . . . . . . . . . . . . . . . 112 


\section{Chapter 1}

\section{Introduction}

Art direction plays a crucial role in visual storytelling in films and games. In a general sense, art direction is referred to as the efforts to maintain a cohesive visual style in these works, including illustrative concept artwork and character designs. For example, the kinds of colours, tones, composition, lighting, and camera angles are all intentionally chosen to convey character traits and the desired moods in the story [5]. Early developments of art direction can be found in live action films since the 50's, such as The Third Man or Rashomon, where fictitious lighting and shadowing were introduced as psychological descriptions of the characters. Such visual elements are carefully adjusted to unify the director's vision throughout the work, and ultimately immerse the audience into the story. Hence, the goal of art direction is not necessarily to achieve convincing reality, but to visually describe a director's unique interpretation of the world.

In this sense, reality is merely one component of art direction. Computer Graphics (CG) technology has been developed since the early 60's with large efforts aimed at achieving photorealism. This has brought unprecedented realism in today's films, games, and digital content industries. Unfortunately, art direction using these tools remains laborious and almost impossible in real-time and interactive content development.

A critical issue in art direction is the task of bringing fictional characters from the artist's imagination into the real world. Before computer animation, films such as 
Mary Poppins, Who Framed Roger Rabbit, and Space Jam used manually composited hand-drawn cartoons in live action film. Today's CG technology allows artists to achieve visually imperceptible insertion of virtual 3D characters into real scenes, by using environment lighting data captured on-set. The problem directors then face is that the true on-set lighting can defy the art direction prescribed for the story. For example, real world lighting can cast undesirable shadows on a subject, or fail to create cinematic lighting styles that support the plot or character traits. While artists adjust synthetic CG lighting to restore art direction shot-by-shot, this is not possible in live interactive applications including games, augmented reality (AR), and mixed reality (MR). These live contexts feature dynamic and varied environment lighting that, when applied to a character, can unpredictably go against the desired style and prior art direction.

This thesis addresses such problems by introducing dynamic art-directed composition for these new real-time media. Traditional film is considered an offline media, due to its pre-rendered and non-interactive nature. Real-time solutions remain relevant for rapid artist iteration, pre-visualisation, and virtual production $[6,7]$. Furthermore, AR and MR applications perform live-action compositing in real time. These platforms differ from offline film compositing, as they estimate the real-world geometry and lighting [8] for a user's current environment, in real-time. This poses a much greater challenge as a user's environment is previously unknown and can change unpredictably. Despite these constraints, new digital media are rapidly taking advantage of the much greater level of interaction and engagement interaction provided by $\operatorname{AR}$ and $\operatorname{MR}[9,10,11]$.

We address the problem of art-directed compositing in new live, real-time, and interactive media (games, AR, MR, etc), where dynamic realistic lighting in these media can disregard art direction. In sections 1.1 and 1.2 we describe the goals of realistic and art-directed compositing, and how they can conflict. Section 1.3 defines the specific problems addressed in this thesis. 


\subsection{Realistic Compositing}

Realistic composition starts with virtual objects described with 3D surface geometry and material properties, either captured or created by an artist. In film, the real-world scene, or background environment, is captured as live-action footage. It is then the compositor's job to achieve seamless blending between real and virtual scenes. We refer to seamless blending or "seamlessness" as the visual illusion of the virtual object coexisting in the real scene. This definition depends on the coexistence between real and virtual, as perceived by a human. It does not necessarily depend on the composition's physical accuracy. This is important for presenting a plausible image to the audience, without any obvious inconsistencies between the two media.

Several stages in the film production pipeline exist to achieve this. Acquiring real-world illumination [12] and geometry [13] is used to simulate shadowing, reflections, and bounce lighting effects between real and virtual scenes. These rendering effects give clear visual cues of a virtual object's existence in a real scene. Simulating this light interaction, given the captured scene information, is standard practice for creating highly photorealistic composites. However, the required scene acquisition steps of illumination and geometry capture are time-consuming and computationally expensive. This cost is also prohibitive for live AR and MR applications, which must capture this scene information in real-time for dynamically changing scenes [14].

\subsection{Art-directed Compositing}

Despite the high photorealism offered by current rendering technology, trained artists manually blend real and virtual elements. Artists are aware of the audience's inability to notice inconsistencies, and exploit this in a composite. A primary use-case for compositing is to insert fictional characters into the real world. Without any real-world reference for this result, they rely on artistic rules and their own judgement to accomplish plausible composites. Brinkmann explains 
that due to the inherent artistic nature of filmmaking, the problem of evaluating a compositing task is a subjective matter where the ultimate rule is "If it looks correct, then it is correct" [15].

Similarly, in The Fundamentals of Animation, Moore and Wells [16] state:

"Compositing is the process of bringing together disparate elements to create a whole on the screen. This can be done to look photorealistically seamless ... or it can be used to creatively to construct a world of the animator's imagination."

This provides the necessary freedom for directors to make stylistic adjustments beyond the physically correct visual result. This idea also accommodates combining non-photorealistic and stylised media with live-action footage, where no physically correct result exists.

Artists and directors frequently compromise between the results of accurate physical simulation, and stylistic motivations of the story and character. Prior art direction defines the intended visual style through character designs and concept artwork. This is critical to visually communicate the director's intended emotional response and aesthetic qualities, regardless of physical accuracy.

For example, lighting artists will art-direct the position of lighting to cast pleasing shadows on a subject, depict shape and material, or convey a specific, mood. The wealth of literature on art and cinematic lighting principles covers these [17, 18, 19] in detail. These often directly conflict with the true lighting the scene, and even change between shots in the same scene [20].

Artists also wish to define different stylised appearances in different lighting conditions. For example, in environments with many lights, artists may opt for controlled top-down [21], or three-point lighting [22] instead of multiple physical but unpredictable shadows. For heavily stylised characters, soft lighting may be depicted with the absence of shading or abstract colour gradation [23], instead of softening shadows like in reality. Per-character lighting styles exist as well. This is important for AR/MR applications exclusively featuring existing cartoon characters [9, 10, 11]. Implausible shadows, uniquely designed for certain 
characters (used to convey personality traits [24]), should also not be affected by physical lighting. These choices depend heavily on art direction and how much a director is willing to differ from the original style in live-action compositing [25].

\subsection{Problem Statement}

This thesis starts by addressing automation for realistic compositing, as it serves as a good starting point for artists. Then, given the strong industry demand for art-directability, we aim to let artists adjust the automatic output to achieve their aesthetic goals. The motivation for this is that professional artists are rarely satisfied by automated results despite their realism. This leaves artists an abundance of manual editing work to be done. Such editing can be afforded during film post-production, but is impossible to apply in dynamic AR/MR environments. Current artistic editing techniques only support fixed lighting environments, requiring re-editing every time the environment changes [26, 27, 28]. We focus on appearance editing that dynamically adapts to the changing real-world lighting environments in AR/MR.

We also target real-time and automatic solutions. Aiming for real-time enables live, interactive applications, such as games, $\mathrm{MR}$, and $\mathrm{AR}$, to preserve art direction. These do not allow for artistic post-production every frame and are in need for automatically applying the stylistic edits that artists would make in different situations. Automation is also beneficial in offline contexts to minimise the need for repeating the same edits, saving artists time. Similarly, real-time capability is immensely time-saving for artists to be able to interactively previsualise and edit renderings in film production.

The objectives to solve these problems, and their relation to each other, are summarised in the following research goals:

1. Our first goal concerns seamlessness (Section 1.1) in realistic $A R / M R$ compositing. To make AR/MR compositing efforts tractable in real-time, previous work optimise image-based lighting and shadowing [29, 14, 30]. However, no previous work optimises the scene geometry needed for 
shadowing and occlusion effects between the real and virtual scenes. This goal is to perceptually optimise the required fidelity in the background scene of a composite. The cost of real-world background scene capture, at high accuracy and fidelity, is prohibitive for live applications. This goal aims to reduce capture costs down to perceptually optimal levels that retain visual seamlessness. Unlike previous work in perceptual level-of-detail, this goal is to find the relationship between real-scene geometry fidelity and visual seamlessness in a composite.

2. The second goal is to automatically preserve artistic intent when real-world environment lighting changes. While Goal 1 concerns the background, the foreground virtual object or character must still match artistic intent. For an automatically art-directed result, the foreground object's appearance must adapt to lighting from different real-world backgrounds, as needed to match art direction. Current techniques can only support editing for one environment and cannot adapt edits between environment changes. Goal 1 and Goal 2 solves automatic perceptual optimisation and art direction in live-action composites.

3. Our final goal is to support art direction for non-photorealistic appearance editing. While achieving Goal 2 helps artists to edit realistic appearance, art direction may call for unrealistic lighting edits for illustrative styles, characters originally drawn in non-photorealistic media, and even clean up realistic shadows [31]. Like in Goal 2, this goal aims to automatically preserve stylised art direction under changing lighting, which is unsupported by existing techniques. This would allow artists to have fine-grained control over shadow shapes, for greater freedom of appearance editing than discussed in Goal 2.

\subsection{Contributions}

The contributions of the thesis, outlined here, address specific issues regarding the research goals: 
- We design and conduct a novel user study to measure how background scene fidelity affects visual seamlessness in realistic composites. We identify perceptual thresholds that optimise scene fidelity while retaining visual seamlessness in a composite. We define categories of composites with different illumination effects between real and virtual elements and measure four thresholds for each case.

- A novel 2D control space that allows artists to art-direct virtual object appearance as real-world lighting varies in a composite. We learn a latent space that encodes various real-world lighting conditions into an artist-interpretable 2D layout. Being 2D, artists can adopt familiar interfaces, such as texture painting to specify and art-direct lighting and material properties, across lighting conditions. Our control space clusters real-world lighting environments into distinct regions and can robustly encode unseen lighting environments into the same layout. This results in artist directives to be automatically preserved when compositing virtual objects into different real-world backgrounds.

- A novel parametric model for art-directable non-physical lighting edits. We review stylised shading literature to distil artistic shadow shapes into mathematical parameters for our model. We show improved animation results over existing art-directable toon shadow animation. Our method is also computationally cheaper than existing shape interpolation methods. This allows artists to define precisely how stylised shading adapts to lighting changes. These artist directives are reproduced automatically in real-time and retain the vector quality required for stylised $3 \mathrm{D}$ rendering.

\subsection{Thesis Structure}

The contributions of the thesis address issues in automating realistic, art-directed, and stylised compositing of 3D content. Chapters 2, 3, and 4, present the work of these thesis contributions independently, with their own introduction, related work, method, results, evaluation, and conclusion sections. The remaining chapters are 
summarised in the following overview:

Chapter 2 gives background theory on differential rendering technique [13], fundamental to compositing shadows. Then we describe the design of a user study to measure how background scene fidelity affects visual seamlessness in a composite. This chapter defines four categories of illumination effects occurring between real and virtual scenes in composition using differential rendering. The measured perceptual thresholds for each case are reported, with a discussion of the findings.

Chapter 3 first introduces using environment maps (EMs) to represent real-world lighting [12]. We then describe the manifold learning approach we use to encode an EM database into an interpretable control space. We examine the resulting space, for consistently learned organisation and clustering. Art-directability examples of using the space to control material and lighting across the latent space are also shown.

Chapter 4 focuses on lower level art-directability with non-physical lighting edits. With a review of artistic shading principles and basic shadow shapes, this chapter gives the design and mathematical formulation of a parametric model for stylised shading edits. We compare with previous work in state-of-the-art 3D toon shading shadow animation and shape interpolation performance.

The 5th and final chapter concludes the thesis by summarising the contributions and a discussion of future work. Supplementary videos that illustrate our contributions and contain animated examples are provided online. ${ }^{1}$

\footnotetext{
1 https://www.notion.so/lohit/LP-Thesis-Supplementary-Material$0 \mathrm{c} 3 \mathrm{f} 104 \mathrm{bc} 25 \mathrm{f} 4 \mathrm{da} 5 \mathrm{bc} 4343632630 \mathrm{bc} 3 \mathrm{~b}$
} 


\section{Chapter 2}

\section{Visual Perception of Real-World Depth Map Resolution for Mixed Reality Composition}

\subsection{Introduction}

The acquisition of depth information from the real world improves various aspects of mixed reality (MR) composition, such as accurately placing virtual objects on real-world surfaces, and supporting mutual global illumination effects. Mutual in this context refers to light transport interactions between the real world and virtual objects. Such interactions include the real-world environment impacting the lighting, shadows and occlusions on the virtual objects, and vice versa. Such illumination effects improve the overall realism of MR compositing, where virtual objects can seem like they naturally belong in the real-world environment. However, a reduction in depth fidelity directly impacts the quality of mutual illumination, where artifacts arise if the depth data is not a faithful reconstruction of the real world.

Obtaining high fidelity depth information from the real world can be a difficult task, involving expensive or time-consuming methods such as LIDAR scanning, photogrammetry, or manual reconstruction by an artist. Alternatively, real time or 
consumer level depth cameras such as Microsoft Kinect can be used to reconstruct the depth information in real-time with the concession of lower fidelity. As such, there is a clear trade-off between fidelity in depth data and depth acquisition, which impacts the quality of mutual illumination. However, it remains unclear the degree to which depth fidelity impacts the quality of the rendered image as observed by the end-user.

Recent work has defined the key notion of a seamless composition, in which virtual objects are believably composited into the real world despite using reduced resources [29]. In order to understand how much real-world depth information is required, we use the notion of a seamless composition with respect to depth fidelity to evaluate various mutual illumination effects. With each of these effects, different artifacts arise, such as protruding geometry or incorrect shadow alignment. A challenge to be addressed is that these artifacts may only violate seamlessness under different depth fidelities. Therefore, it is important to isolate each artifact and evaluate them independently.

To our knowledge, no prior work has addressed this specific problem. Closely related previous approaches have addressed visual quality of virtual geometry, such as level of detail (LOD), but do not evaluate it in the context of a seamless composition in MR compositing. Other approaches evaluate composition quality, but do not consider depth fidelity in relation to composition directly. In this chapter, we conduct a user study that evaluates depth fidelity with respect to mutual illumination. Adhering to the notion of a seamless composition, we find perceptual thresholds of depth resolution in a MR context. Through this process, we mitigate common artifacts by choosing appropriate levels of depth fidelity. Figure 2.1 illustrates this process.

The main contributions of in this chapter are summarised as follows:

- We design and conduct a novel self-referencing user study to evaluate the depth fidelity required for MR compositing including mutual illumination.

- We investigate perceptual thresholds for MR compositing by defining four composition artifact types based on depth, and evaluating each type independently. The types include virtual shadows cast onto the real world, 


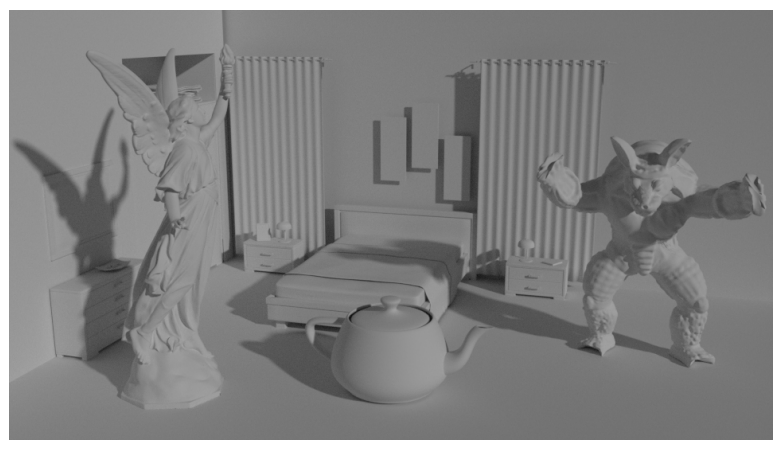

(a) Reference

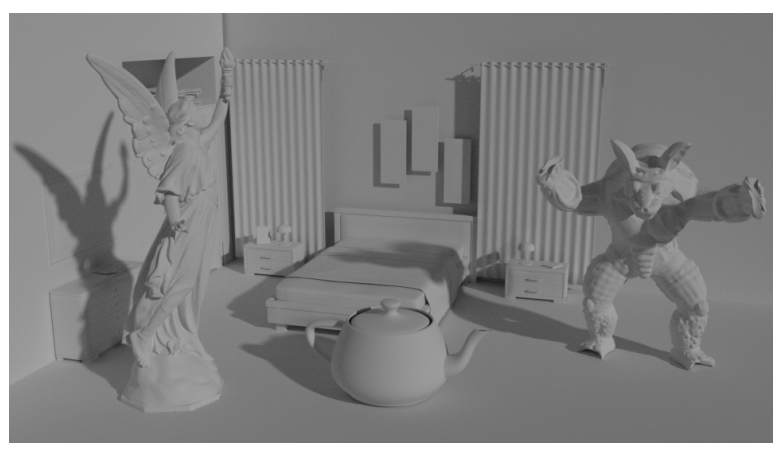

(c) $426 \times 213$ (optimal)

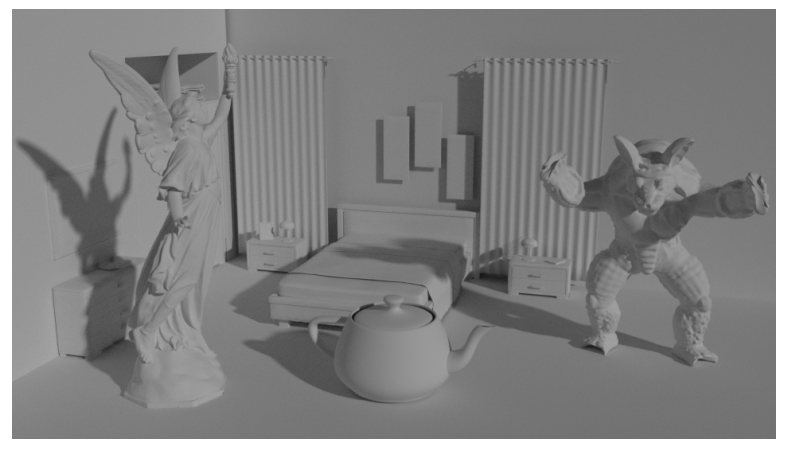

(b) $4096 \times 2048$

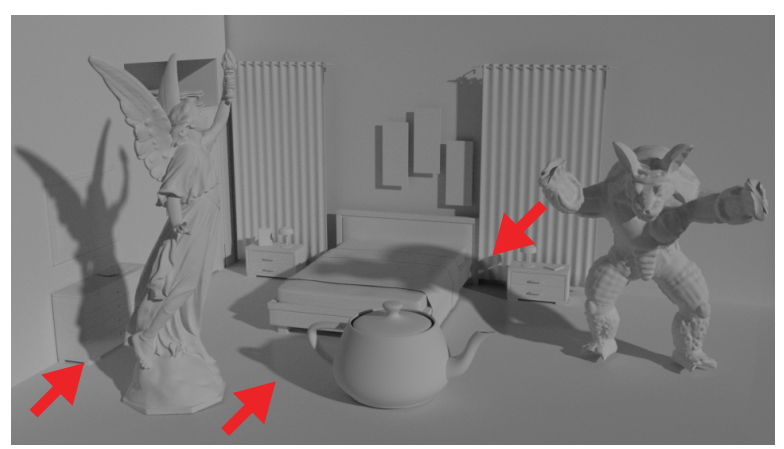

(d) $64 \times 32$ (visible artifacts)

Figure 2.1: Examples of compositing foreground objects (statue, teapot and armadillo) into the background (Bedroom scene) using its depth map. Comparison between ground truth reference rendering with high resolution 3D meshes (a), composition with high resolution depth map $(4096 \times 2048)(b)$, composition with perceptually optimal depth resolution $(426 \times 213)(\mathrm{c})$, and composition with insufficient depth resolution $(64 \times 32)(\mathrm{d})$ showing noticeable artifacts from poorly reconstructed geometry. 
real shadows cast onto virtual objects, the real world occluding virtual objects, and overlapping of real and virtual shadows.

- From our experiments we find low perceptual thresholds for depth resolution for mutual shadowing artifacts. We show that low depth fidelity is sufficient for perceptually similar composition, coarse 3D geometry modelling and estimation of depth is an alternative to accurate depth capture.

\subsection{Related Work}

\subsubsection{MR compositing with Depth}

Seamlessly blending real and virtual worlds has been an ongoing problem in computer graphics. Debevec [13] achieved seamless rendering of virtual objects into photographs using captured illumination data from the real world, and compositing via differential rendering. Local scene geometry is used to simulate occlusion and shadow interaction between real and virtual objects. This geometry is often manually modelled to approximate real shadow receivers [14]. For complex scenes, however, these approximations are either too coarse, or it is time consuming to create an accurate geometric representation.

AR applications have used depth cameras to capture RGBD data for live reconstruction of a dynamic real scene [32, 33, 34]. Karsch et al. [35, 36] automate scene geometry acquisition by inferring a depth map from the given photograph. This achieves realistic composition of shadows and reflections onto the scene while foreground occluders are provided by user input. In visual effects, photogrammetry is a common but resource intensive acquisition method for high quality geometry [37]. Deep compositing also incorporates depth data into visual effects pipelines but does not address mutual lighting artifacts exhibited in rendering [38].

All of these techniques trade off time, accuracy, artist effort, and hardware cost. It is not clear which method of depth acquisition is required for a given composition problem. Previous work by Jacobs et al. [39] uses a coarse geometric model of 
real objects in AR, and illustrates artifacts that arise. They address certain shadow artifacts by offloading computational effort towards shadow estimation rather than geometry refinement. Such work supports the hypothesis that capturing of low resolution depth could be sufficient. Likewise, measuring the noticeability of these artifacts relative to each other would be useful for these applications, which is the focus of is chapter.

\subsubsection{Perceptual Studies with Depth}

Depth perception studies have measured the human visual system's (HVS) ability to make distance judgements with depth cues [40], and infer spatial relationships between virtual objects in AR [41,42]. Depth perception has also been evaluated against a display's ability to convey depth cues [43, 44, 45]. Depth cues on more limited displays have been simulated with depth-of-field blur [46]. Other studies measure the effect of depth-of-field blur on depth perception [47]. Our studies instead focus on properties of reduced depth map data that pertain to $\mathrm{MR}$ composition.

More related to the geometry estimation problem, reduced level of detail (LOD) has been well researched for optimisation of $3 D$ triangle meshes $[48,49]$. In particular, perceptual factors such as visual acuity and gaze are often considered to obtain simplified geometry that produces visually similar renderings [50]. Corsini et al. [51] provide a survey of user studies for perceptually based error metrics for mesh distortion in both static and dynamic scenarios. These works aim to preserve perceptually important details such as texture, object silhouettes, and rendering, in fully virtual scenes. Our studies instead measure how well geometry estimation can preserve perceived lighting interaction between real and virtual objects in MR. Sugano et al. [52] show that shadows are important for believable composition, in terms of virtual object presence and spatial cues. In their perceptual studies they use static modelled geometry for shadows to be cast on the real world, but do not vary the geometry in a photorealistic composition setup. So far, no other works perceptually evaluate the effect of depth approximation in a MR setup. 


\subsubsection{Perceptually Based Rendering}

Perceptually based rendering exploits limitations in the HVS by avoiding computation of rendering effects that are not perceived in the final rendering [53]. In virtual scenes, visibility approximations have been shown to be sufficient for rendering perceivably accurate indirect illumination [54]. Preliminary studies have shown that soft shadows can be rendered with blocker geometry of lower LOD than the actual blocker object [55]. While their shadows were cast from synthetic objects in a virtual scene, it supports the idea that real geometry could be approximated for soft shadowing of virtual objects in MR composition.

Chalmers et al. [29] showed that virtual objects can be rendered under low resolution radiance maps and maintain seamlessness in its shading and shadow. Iorns and Rhee utilise this work for perceptually optimised image based lighting with low resolution, low dynamic range $360^{\circ}$ video for real-time MR object shading [30]. Further studies by Rhee et al. [14] use perceptually based thresholding to parameterise lights in $360^{\circ}$ videos. With this notion of perceptually optimised illumination, we apply this concept to depth for finding optimal depth map resolutions for MR.

\subsubsection{Perceptual Studies in Composition}

In many previous perceptual experiment frameworks, composition quality is evaluated against a ground truth reference image. The Visual Equivalence [56] metric has been used to accept visually similar images, more so than the Visual Differences Predictor [57] which can still detect imperceivable differences. Křivánek et al. [58] has used this to evaluate HVS sensitivity to common global illumination rendering artifacts. MR applications have also been evaluated by ground truth comparisons $[59,35,60]$.

However, often the motivation of MR is to insert objects that do not belong in the same environment as the background. Therefore, we use a framework that evaluates visual coherence and plausibility within the same image, as the ground 
truth reference does not always exist in practice. We adopt the idea of a self-reference perceptual experiment frameworks used by Chalmers et al. [29] and Rhee et al. [14].

\subsection{Composition with Depth}

Before the experimental setup is explained, this section reviews differential rendering composition and how we integrate depth maps in the process. We then explain and label the artifacts that arise when using approximate depth in composition.

\subsubsection{Differential Rendering}

The Differential Rendering method [13] is used to composite the shadows of virtual objects into photographs. This method requires a geometry and material model of the surrounding real environment, which is referred to as the local scene. The local scene is rendered twice: once with the virtual objects and once without. The perpixel ratio between the renderings extracts the change that the objects imparted onto the local scene (e.g. shadows, reflections, and global illumination). These changes are then composited into the background as follows:

$$
I_{\text {final }}=I_{\text {Background }} \frac{L S_{O b j}}{L S_{N o O b j}}
$$

In Equation 2.1, $I_{\text {final }}$ is the final composite, $I_{\text {background }}$ is the real background image, and $L S_{O b j}$ and $L S_{N o O b j}$ are the local scene renderings with and without objects, respectively. The ratio $L S_{O b j} / L S_{N o O b j}$ is multiplied with the background so that shadows (ratio $<1$ ) darken the image and bounce lighting (ratio $>1$ ) brightens it. Figure 2.2 shows this process. Taking the ratio between renderings and multiplying the result is a variation of differential rendering proposed by Debevec [13]. We use this instead of the more common additive method, which was found to produce noise and clipping artifacts. The local scene is used to receive their shadow detail 
as if cast onto the real scene. Therefore, the accuracy in the interaction of light, shadow, and occlusion between real and virtual objects is heavily dictated by how closely the local scene represents the real environment.

Many applications acquire the local scene model via depth maps either captured by depth cameras $[32,33,34]$, or estimated from the image [35, 36]. Aside from fundamental limitations such as disocclusions (holes) [61], high resolution depth maps can generate a local scene model for composition with correct shadowing and occlusion between real and virtual objects.

\subsubsection{Local Scene Generation from $360^{\circ}$ Depth Maps}

Our studies use $360^{\circ}$ panoramic depth maps to preserve out-of-view geometry, thus avoiding inconsistent illumination artifacts which are not being tested. Out-of-view geometry impacts illumination in the final composite even in conventional fixed-view output. $360^{\circ}$ equirectangular depth also allows the depth map resolution parameter to be varied independently of the camera's field-of-view (FOV).

Given a $360^{\circ}$ panoramic depth map in equirectangular format, a mesh is generated from it by creating a high-density spherical mesh, centred at the origin. We set each vertex's length equal to the depth map value sampled by the vertex's direction. For low resolution depth maps, we linearly interpolate the depth samples across the mesh vertices. Using a mesh mitigates artifacts such as holes from other depth image rendering methods such as point clouds.

The generated depth mesh becomes the local scene geometry in differential rendering. Figure $2.2 \mathrm{~b}$ shows the geometry generated with this method using a $512 \times 256$ depth map, with visible loss of high frequency details in the original scene. This simple approach was sufficient for our experiment but other mesh generation methods such as Pajarola et al. [62] would be valid as well. 


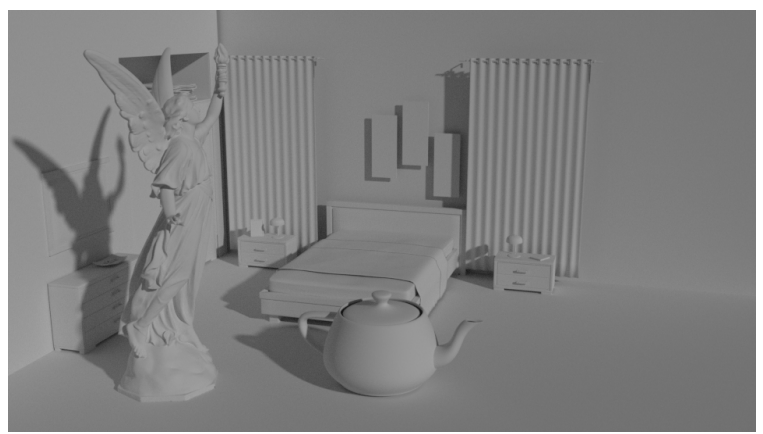

(a) Background

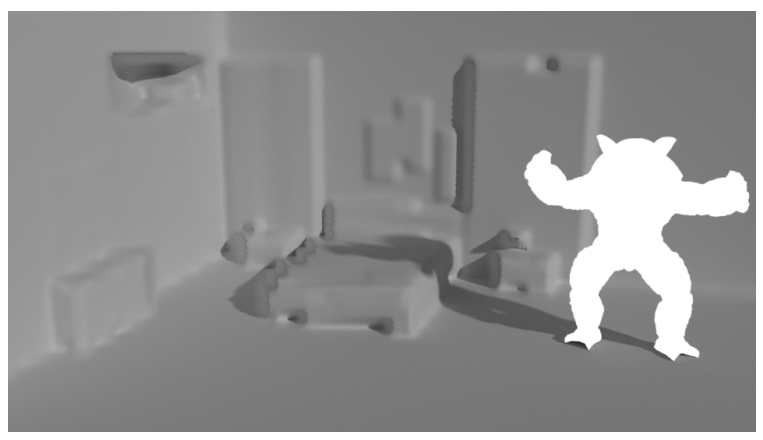

(c) Local scene with object

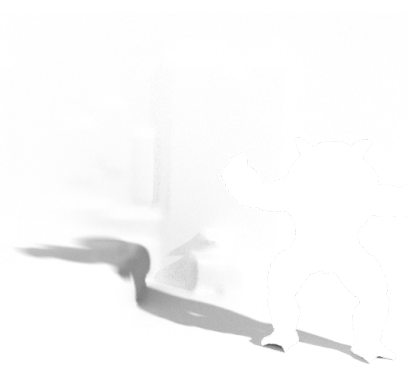

(e) Local scene ratio

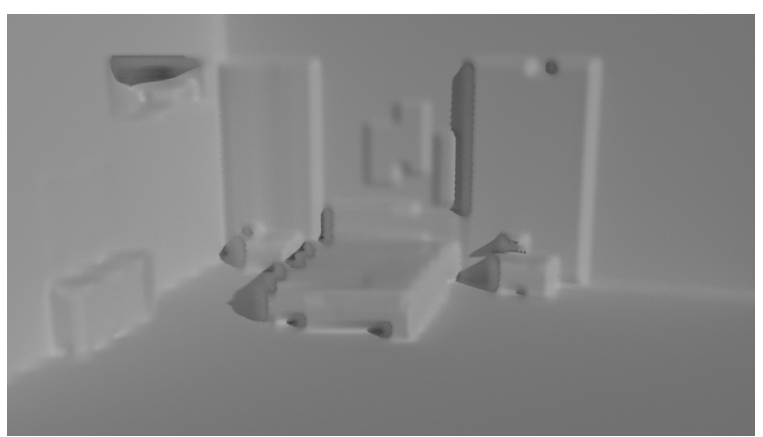

(b) Local scene

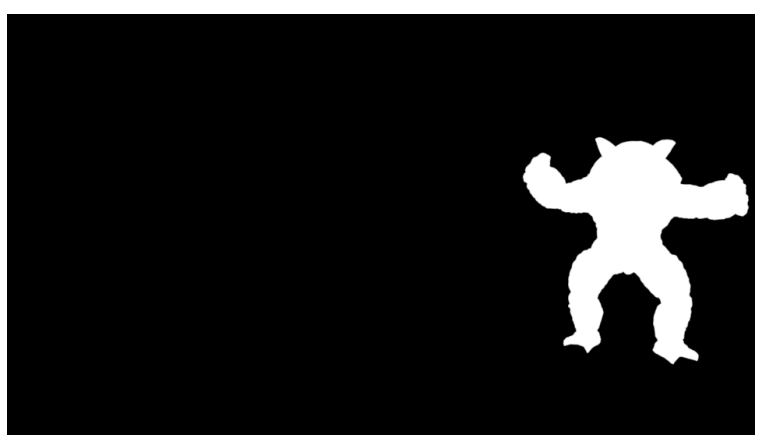

(d) Object matte

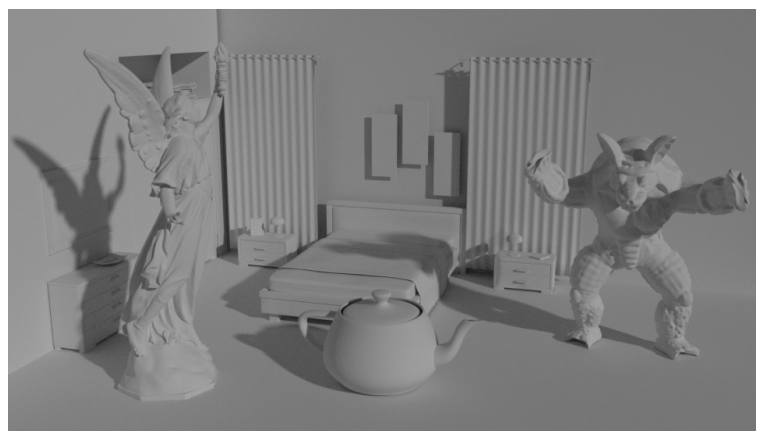

(f) Final composite

Figure 2.2: Compositing steps for differential rendering. (a) Background image, (b) Reconstructed local scene mesh from depth map, (c) Local scene rendered with virtual object, (d) Object matte, (e) Change in local scene: (c) divided by (b) excluding object, (f) Final Composite with object and its shadow. 


\subsubsection{Depth Composition Artifacts}

As the depth map degrades in resolution, specific artifacts are observable in the final composite and occur in different types of scenes. We choose a subset of artifacts to evaluate. Based on our preliminary pilot studies, we found that four artifact types were perceived to be most noticeable in depth-based compositions. We also noticed that each artifact became noticeable at different depth resolutions. Therefore, our experiments were designed to study these four artifacts individually. Figure 2.3 shows how each artifact changes in appearance with varying depth resolutions. We define and explain each artifact type in the following.

Type 1 - Real objects receiving virtual shadows: Geometry reconstruction from low resolution depth maps fails to fully reconstruct high frequency details in the real objects in the background image. A virtual object's shadow received by this geometry will hence be distorted in shape, and not match the background in the composite.

Type 2 - Virtual objects receiving real shadows: Similarly to Type 1 , shadows cast from the real world geometry onto virtual objects will be inconsistent when low resolution depth is used. This is due to the reconstructed real blocker being distorted, thus casting a different shadow on a virtual object.

Type 3 - Occlusion boundary: This artifact is seen when virtual objects are composited behind real objects and the occlusion boundary is misaligned with the real image. When depth data is used in the composition, depth testing can be done between virtual and real depth values. From this we obtain a visibility mask allowing the real world to occlude virtual objects in compositing. However, low resolution depth maps fail to create occlusion boundaries that exactly match those in the background image.

Type 4 - Real and virtual shadow overlap: This artifact is seen when virtual shadows over-darken the background real shadow, even though the visibility in that region has not changed. When low resolution depth maps poorly reconstruct real world shadow casters, the real shadow simulated during rendering will poorly reconstruct the same shadow region seen in the image. When a virtual object 
shadow also falls in this region, an artifact of double shadowing or shadow overlap will be present in the composition. The simulated real shadow dictates where virtual object shadows should not darken the background since $L S_{O b j}$ and $L S_{N o O b j}$ are both dark. Therefore, the artifact is seen where the simulated real shadow does not fully cover the real shadow in the background. This is illustrated in Figure 2.4.

These four artifact types are the scope of our experiments. The next section details the design of our experiment which isolates the artifacts to analyse their noticeability independently.

\subsection{User Experiment}

The aim of our user experiment is to measure the effect of depth map resolution on seamlessness, while finding the optimal resolution thresholds. Through pilot studies we found that some participants noticed artifacts more or less depending on different composition cases in scenes with the same depth fidelity. We also tested different methods of scene representation optimisations such as depth layers. Depth layers produced accurate occlusions but unrealistic shadows, so the depth maps were chosen for our studies. Our pilot studies have shown that some of the four artifact types (described in Section 2.3.3) are more noticeable than others. Therefore, our goal was to find multiple resolution perceptual thresholds to enable applications to target different composition cases independently. The study was taken by 20 participants (five female, 15 male) between ages 20 to 52 . All participants had normal or corrected to normal vision. Out of the 20 participants, 13 were either students, developers, researchers, or artists working in technical fields such as computer graphics, computer science, or computer vision. The remaining seven had other non-technical backgrounds. We distributed the test as an online survey through Google Forms. 


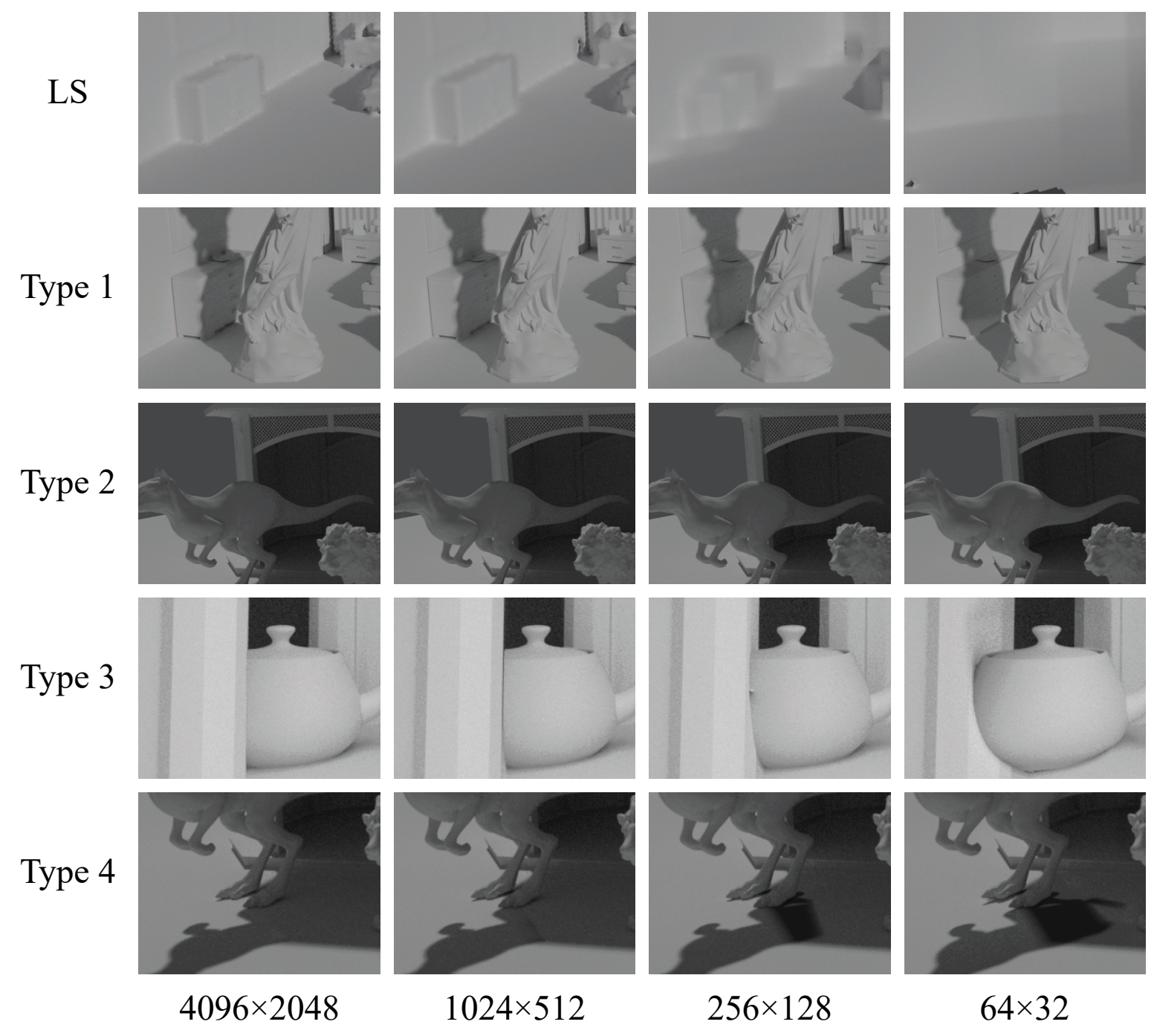

Figure 2.3: Artifact types and their behaviour as depth map resolution decreases. The bottom labels indicate the depth resolution used in each column. The top row (LS) shows the Type 1 scene's geometry reconstruction as an example. The decreasing depth map resolution causes the geometry to degrade, creating the artifacts in Type 1 . 


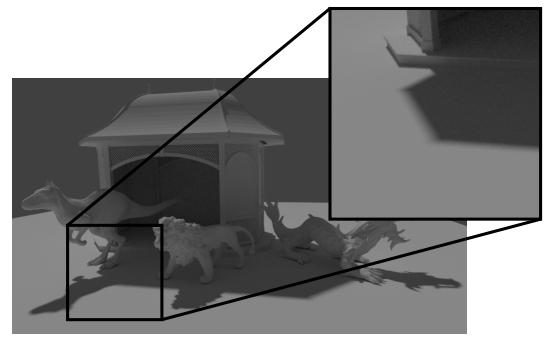

(1)

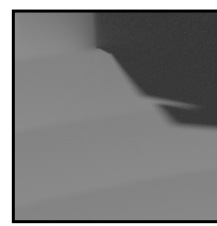

(2)

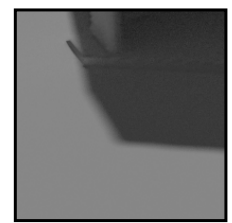

(a)

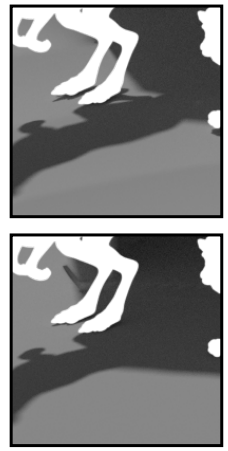

(b)

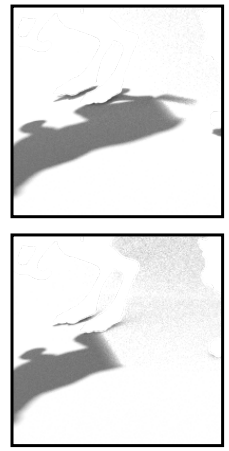

(c)

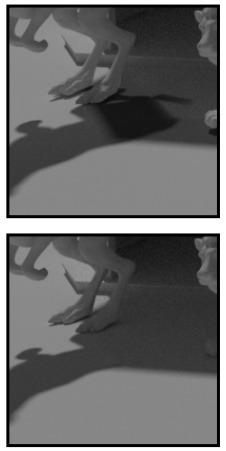

(d)

Figure 2.4: How the Type 4 shadow overlap artifact occurs in differential rendering. The left image and close-up are the background image. Column (a) is the local scene, (b) is the local scene with objects, (c) is the error, and (d) is the final composite. Row (1) shows the process using a $64 \times 32$ resolution depth map, while row (2) uses $4096 \times 2048$.

\subsubsection{Stimuli}

Users were shown images of virtual objects composited into fully synthetic scenes, rather than real photographs with $360^{\circ}$ depth. This is partly due to the lack of available datasets with $360^{\circ}$ panoramas with both high dynamic range (HDR) radiance (as required for photorealistic rendering [13]) and corresponding accurate depth. Furthermore, this would ensure that depth resolution effects are evaluated independently from illumination accuracy. It also allowed us to control camera placement to ensure the artifacts were clearly exhibited in the composites for a meaningful user response. Although $360^{\circ}$ depth data was used, users were shown fixed-view composites to direct focus towards the region of interest with virtual objects and artifacts.

To simulate MR compositing with depth, we convert the synthetic scenes' 3D mesh geometry into a depth map by rendering their depth to an image. Composites were created using the method described in Section 2.3, where equirectangular depth maps were rendered along with the background image. The synthetic depth meshes were then generated from the depth maps and were used as the local scene for each composite. With known illumination and camera projection used to render 
the background, the virtual objects were then rendered under the same conditions with only the local scene model changing with depth map resolution.

All images were rendered at $960 \times 540$ resolution. The $360^{\circ}$ depth map resolutions were chosen to be $64 \times 32,128 \times 64,256 \times 128,512 \times 256,1024 \times 512,2048 \times 1024$, $4096 \times 2048$. This choice of range was informed by preliminary pilot studies. These depth maps were re-rendered from the original 3D scene (as in Figure 2.1a), at the specified resolutions rather than downscaled from a high resolution depth map. This introduces aliasing in the depth maps but avoids filtering to preserve accurate depth measurements. It also simulates capturing from low resolution depth cameras that cannot filter across multiple samples.

\section{Scenes}

To measure each artifact type independently we used three test scenes in the stimuli. The scenes cover each mutual illumination situation that exhibits the type being evaluated. We refer to the renderings of these scenes as the backgrounds in the compositions. The objects placed in them that exhibit the artifacts are referred to as the foreground objects. Figure 2.5 shows each scene and illustrates the artifacts in the stimuli shown to participants. We have kept the scenes simple to exhibit only the relevant artifacts, while avoiding the influence of factors that were not being tested. For example, all scenes were entirely diffuse to avoid reflection inaccuracies in the virtual objects. Similarly, all materials were the same colour to avoid sensitivity variation with different colours.

The Bedroom scene (Figure 2.5a), which showed Type 1 artifacts, has background cuboid geometry and walls, with the foreground objects Lucy, Teapot, and Armadillo. The shadows of the foreground objects are cast on various surfaces such as the floor, bed, and walls. The requirement of this scene's design was to ensure that shadows did not solely fall on trivial surfaces such as large planes which do not exhibit Type 1 artifacts as depth resolution decreases.

The Gazebo scene (Figure 2.5b) showed both Type 2 and Type 4 artifacts by having a large background Gazebo whose shadow partially falls on the foreground objects Killeroo, Lion, and Dragon. This arrangement provided Type 2 shadows falling on 
objects, and Type 4 shadow overlap artifacts where the object and Gazebo shadows met. To test these two artifacts independently, we masked out the Type 2 artifact in one set of stimuli images, and masked out Type 4 in the other set.

The Sponza scene (Figure 2.5c) had teapots occluded behind pillars in the environment to show Type 3 artifacts. During rendering indirect light would normally bounce behind the pillars and illuminate the back walls. After generating the depth mesh, the indirect light would be blocked due to depth disocclusions (cannot store pillar and the wall behind in a single depth map). Hence, objects placed behind the pillars would be dark in the composition. We therefore needed to replicate this in the scene by placing geometry behind pillars, before rendering the background. This prevents indirect light passing behind pillars, thus matching the reconstruction. This enabled the Sponza scene to eliminate other illumination artifacts so that the HVS response only depended on the Type 3 artifact.

\section{Objects}

The Bedroom shadow scene used tall objects (Lucy and Armadillo) under a light with low elevation in order to cast long shadows. These interact with the background across a sufficiently visible area to clearly present any composition artifacts in the stimuli. The teapot is an exception being much shorter but was needed to keep the Armadillo shadow visible in the image. The Gazebo scene objects needed to be long enough to be partially in and out of shadow. The Gazebo casts a shadow onto the objects and using long objects ensures that the shadow edge is clearly visible. Similarly, the overlap in the object shadow and Gazebo shadow must be visible on the ground. The light direction was adjusted from behind the Gazebo such that it shadowed the objects in this way. Different objects were used in Bedroom and Gazebo scenes to prevent participants relying on pattern matching of artifacts to identify the composited objects. The Sponza scene used the same teapot objects but they were placed behind different occluders for artifact variation.

To fairly test shadow artifact noticeability, all objects and their shadows were equally visible from the camera's perspective. Similarly, although all composites had the same resolution, the relative distance and scale between objects and camera were 

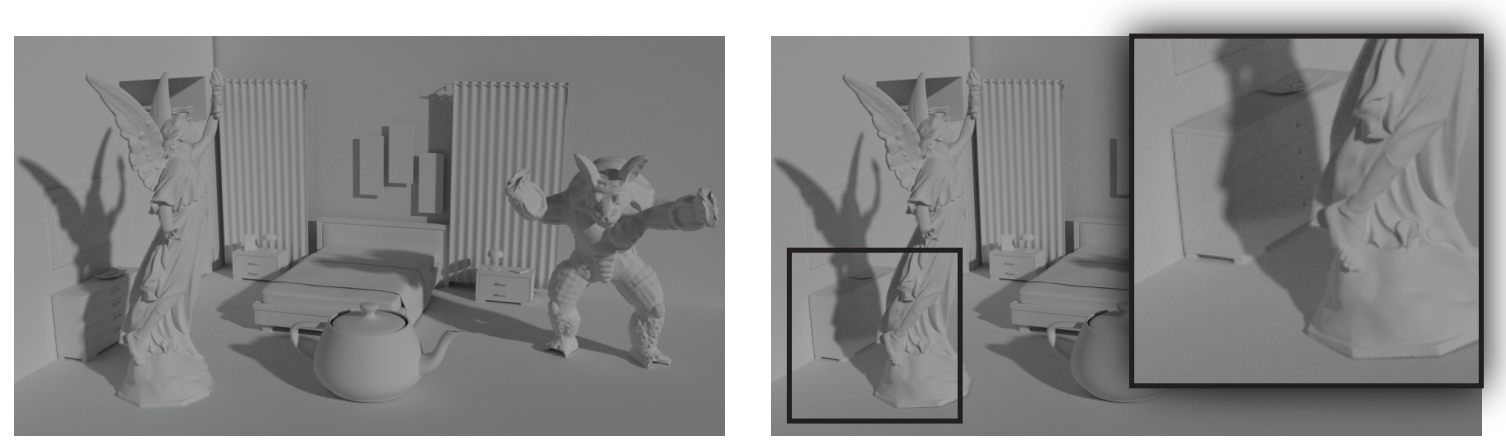

(a) Type 1 - Bedroom
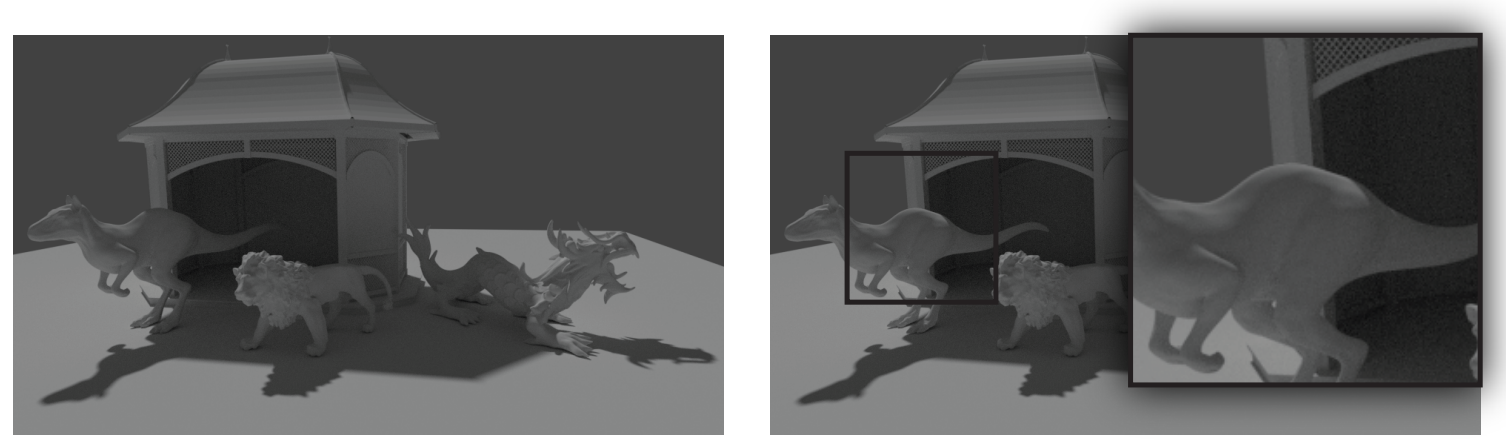

(b) Type 2 - Gazebo
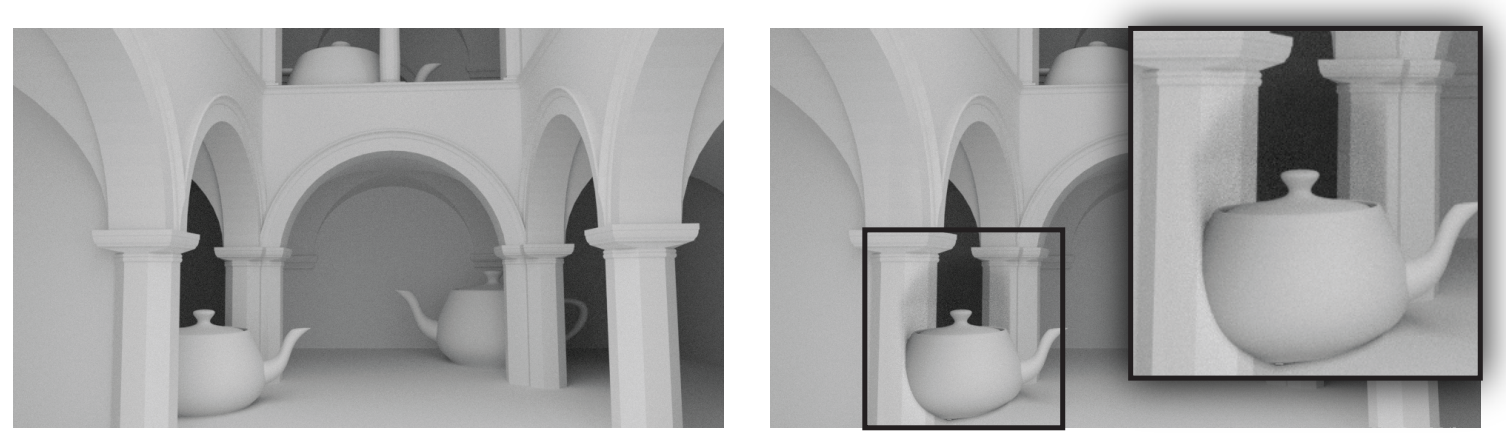

(c) Type 3 - Sponza
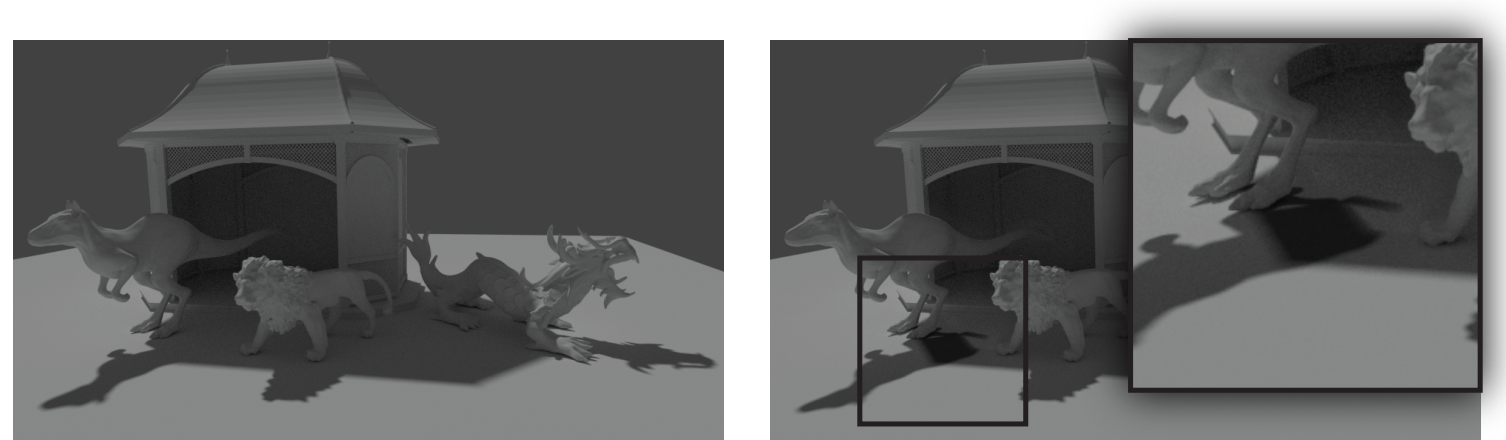

(d) Type 4 - Gazebo

Figure 2.5: Top row: ground truth renderings of the scenes used in our experiment. Bottom row: example stimuli with the left most object being the inserted object in each scene. Type 2 (c) and Type 4 (d) share the same scene but with different artifacts masked out in the composition. 
consistent within scenes. This imposed restrictions on the type and placement of objects such as the teapot exception in the Bedroom scene. Therefore, any object could be used as long as they exhibit the artifacts being evaluated. Objects within Bedroom and Gazebo had consistent distances away from the camera, to test artifacts independently from distance. While Sponza had objects at different distances, their screen-space sizes were equalised. Plausible camera viewpoints were chosen such that these requirements were met and all objects and their artifacts were visible.

\section{Illumination}

Soft shadows were found to be too faint around composition artifacts and were thus unable to stimulate the desired user response across the shadow artifact types. It has also been shown that composition artifacts are much less noticeable under soft lighting compared to hard lighting [29]. Therefore, hard directional lighting was used to create sharp shadows that clearly exhibited the artifacts caused by shadow composition.

Constant colour environment lighting was present in all scenes. This was used to ensure shadows were not too dark. This was required for perceiving the Type 3 shadow overlap artifact because no overlap can be seen when both shadows are pure black.

The Sponza scene, however, used only the ambient occlusion rendering pass for lighting. This was to eliminate any hard shadow artifacts from directed lighting and object illumination artifacts from unreconstructed depth. This ensured that this test only evaluated Type 3 artifacts instead of the other shadow artifacts, while keeping the images realistic.

\subsubsection{Procedure}

Measuring visual equivalence with a reference can assess the perceived accuracy to which composition matches a ground truth rendering. Instead, we aim to measure the perceived seamlessness of composition into a given background image. Users were shown compositions of foreground objects in the previously described 
backgrounds, where one of the three foreground objects were composited with the depth map instead of rendered with the environment. Users were then asked which of the three was the composited object. For each object they were asked "How noticeable was the insertion artifact?" with the Likert item ratings "1. Slightly noticeable", "2. Moderately noticeable", "3. Very noticeable", and "4. Extremely noticeable". If the user instead chose a real object, the default noticeability of 0 was given. Users were forced to choose a composited object even when it was too difficult to identify. This means that responses where users picked the correct object by chance, with noticeability of 1 , will introduce a bias towards the artifact being noticeable in the results. However, this forced choice does prevent users from frequently relying on a default option, improving the usability of the data. In total, 84 images were shown to each participant (7 depth resolutions $\times 3$ objects $\times 4$ artifact types).

\subsection{Results and Analysis}

We firstly average noticeability values from user responses across objects, at each $360^{\circ}$ depth map resolution and for each type. In Figure 2.6 we plot the relationship between average artifact noticeability and depth resolution. Specifically, we plot noticeability against the depth map width, as each depth map's height was half its width. The error bars show the standard error of the mean (SEM) indicating relative error size between points. These plots indicate that noticeability decreases with resolution, and is overall consistent across objects and scenes, as expected. It also verifies that the choice of scenes, objects, and placement were suitable for showing the expected behaviour of higher noticeability at lower resolutions.

To find meaningful perceptual thresholds for each artifact type, we average noticeability values across objects and plot this variation with depth resolution in Figure 2.7. The error bars show each point's SEM. The dashed line at noticeability = 0.5 is halfway between the artifact being noticeable (1.0) and it being not noticeable (0.0). Average noticeability values above 0.5 can distinguish that over $50 \%$ of those artifacts were noticeable, instead of not noticeable (users guessing). From this line we obtain the perceptual resolution thresholds for Types 1 and 2, though there are 
Type 1 - virtual shadow

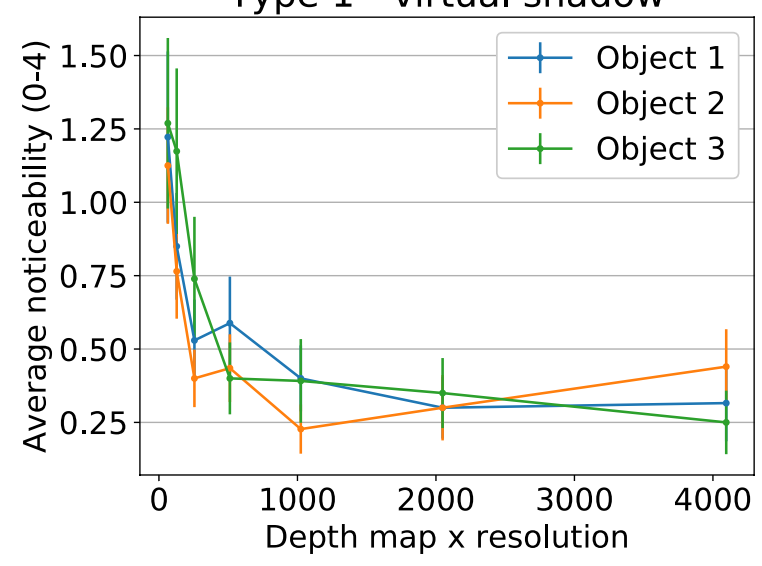

Type 3 - occlusion

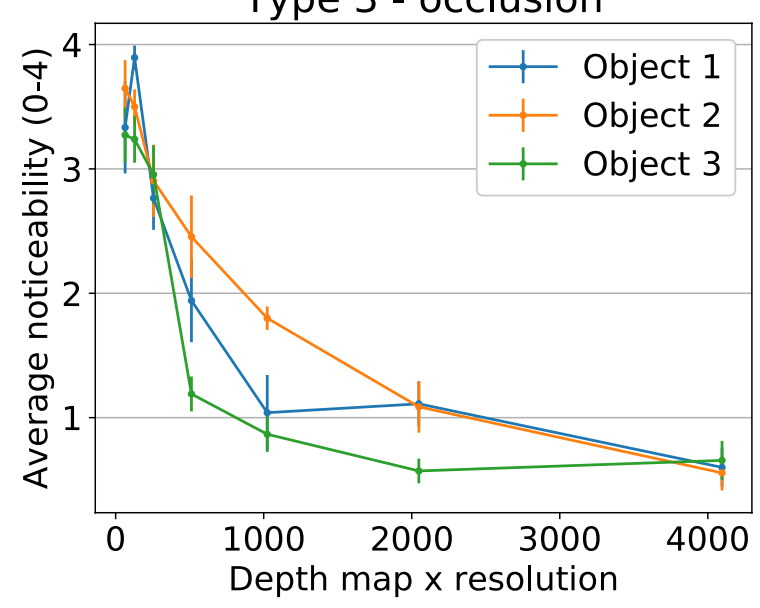

Type 2 - real shadow
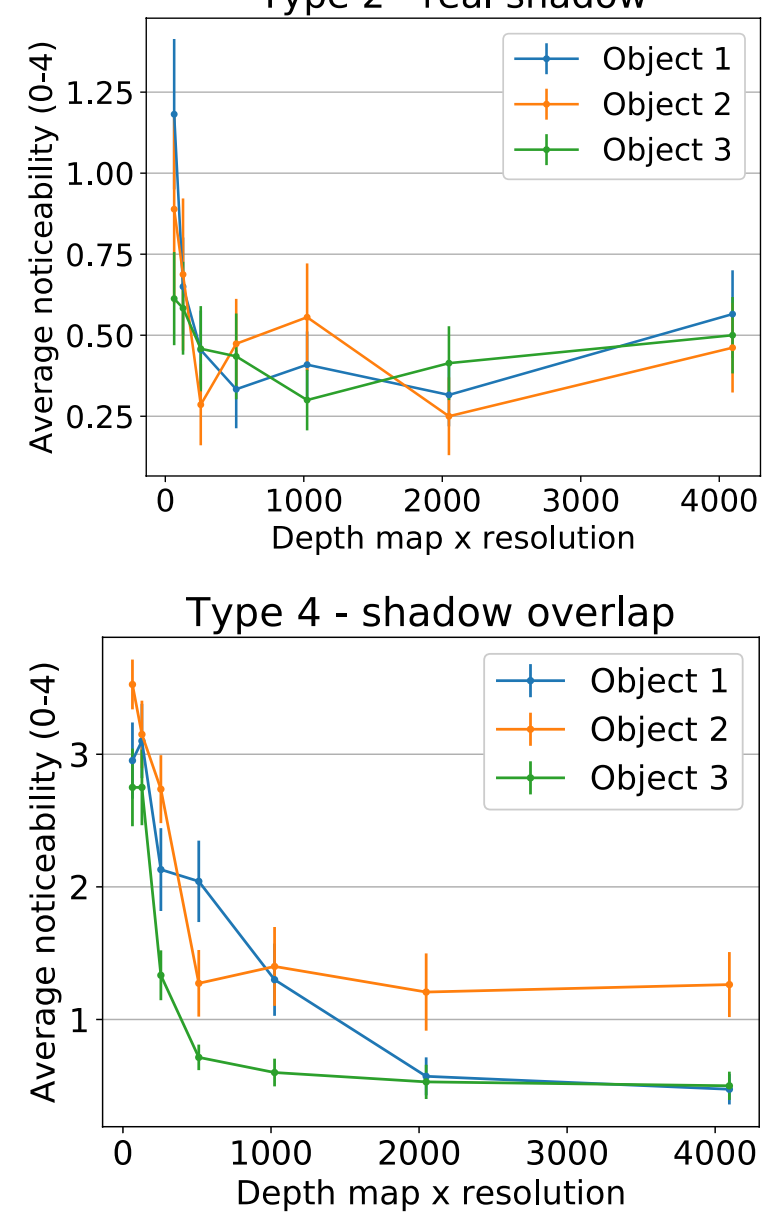

Figure 2.6: Experiment results of artifact noticeability across all types and objects, against depth map width (height is half width). Lower noticeability values are better. Objects 1, 2 and 3 are the left, middle and right objects, respectively, in each Type's scene. 


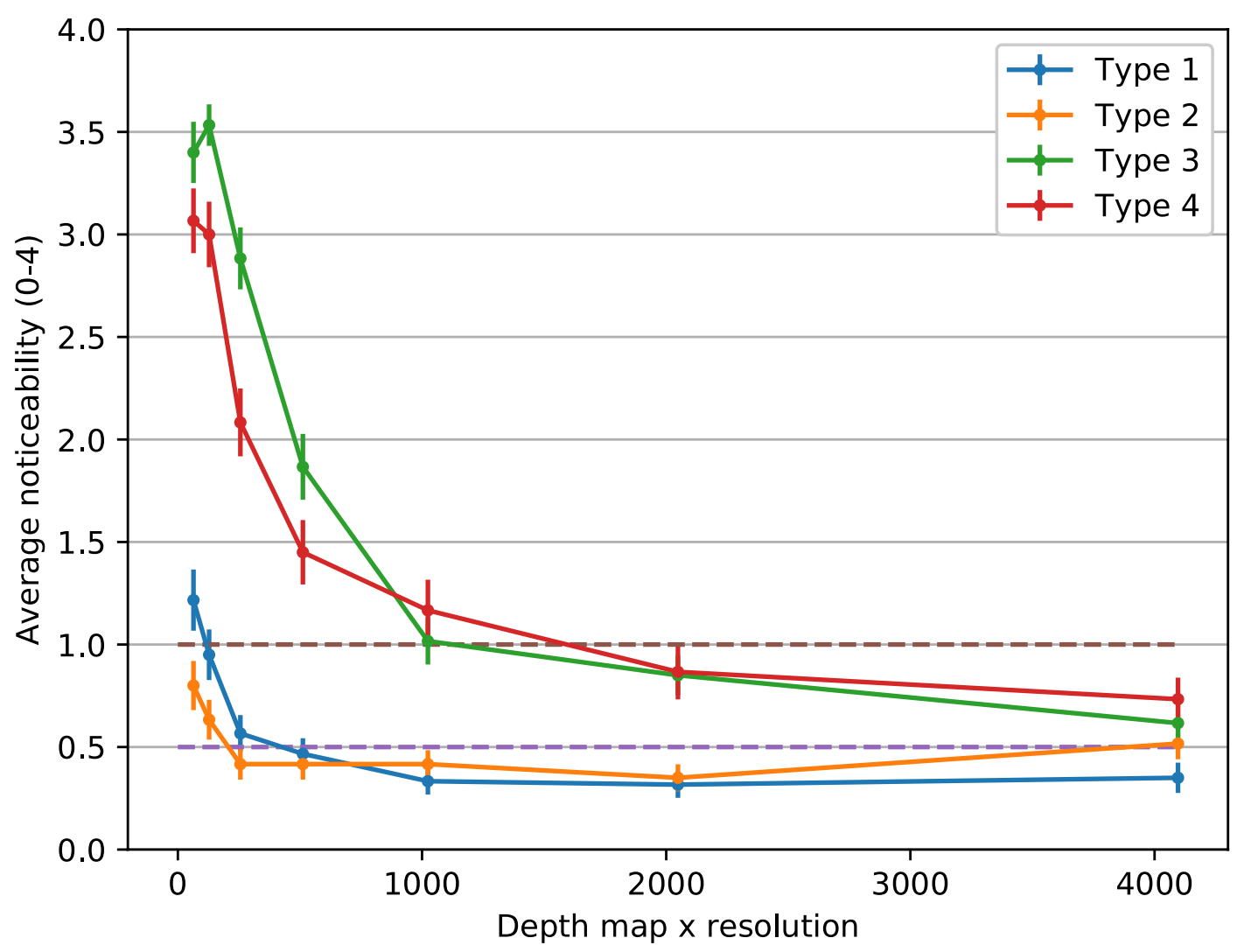

Figure 2.7: Average artifact noticeability against depth map width, for all types. The dotted line at noticeability $=0.5$ indicates where the perceptual thresholds are determined. The dotted line at noticeability $=1.0$ indicates where artifacts become slightly noticeable. Lower noticeability values are better. 
none for Types 3 and 4 within the tested range. As mentioned, users guessing the correct object by chance will bias the results towards higher noticeabilities. Therefore, we also plot a weaker noticeability line at 1.0 to account for this. The thresholds measured at this line indicate where artifacts become slightly noticeable, corresponding to the response option in the survey. We also analyse inflection points of each curve. These points indicate the resolution at which the noticeability starts to increase the most. The perceptual thresholds and inflection points are given in Table 2.1. Analysis methods such as ANOVA and t-test calculation are not suited for our experiment design as we measured gradual change rather than distinct groups with which to measure differences in means.

Table 2.1: Table of depth map resolution perceptual thresholds (PT), slightly noticeable thresholds (SN), and inflection points (IP) from experimental results.

\begin{tabular}{|l|l|l|l|l|}
\hline & Type 1 & Type 2 & Type 3 & Type 4 \\
\hline PT & $426 \times 213$ & $206 \times 103$ & - & - \\
\hline SN & $116 \times 58$ & - & $1126 \times 563$ & $1596 \times 798$ \\
\hline IP & $250 \times 125$ & $228 \times 114$ & $354 \times 177$ & $336 \times 168$ \\
\hline
\end{tabular}

The inflection points for Type 1 and 2 closely correspond to their perceptual thresholds, further validating the position of the thresholds. While there are no perceptual thresholds for Types 3 and 4, within the measured range, the slightly noticeable thresholds can be found for Types 3 and 4, but not Type 1. All inflection points indicate the optimal design trade-off for quality and resolution for each type.

A level of noise is seen across all plots, causing some artifacts at high resolutions to be more noticeable than at lower resolutions. We attribute this to depth map aliasing as the resolution is reduced without filtering (for accurate depth). Each aliased version sampled the depth map differently, such that artifacts are coincidentally hidden at lower resolutions. The averaged plot in Figure 2.7 reduces the noise but it is still seen between the first two points of Type 3, and across Type 2. 


\subsection{Applications}

From our results we provide a number of perceptual thresholds that can be used by artists to understand which areas in the real background require high quality geometry recreation, and which require coarse modelling. Developers can use this understanding to balance computational resources to trade-off performance and quality as required. Researchers can also use the results to develop new perceptually optimised depth acquisition algorithms or depth cameras specifically for MR.

One practical application for the Type 1 result is that, since its perceptual threshold is low, it only requires coarse recreation of surrounding geometry which saves resources such as memory and computation. Coarse box model estimation of geometry $[63,64,65]$ is also sufficient, as the Bedroom test scene composition shows, that can seamlessness is achieved without fine details on large background objects and walls.

Figure 2.8 shows the Type 1 perceptual threshold being used in practice, in a MR composite of virtual objects into a photorealistic, rendered background image. This simulates compositing into a real photograph with a captured $360^{\circ}$ depth map. We illustrate that composition using a depth map at our perceptually optimal threshold yields visually similar results to using a high resolution depth map. Figure 2.8 also shows a limitation in which artifacts arise from discontinuous depth (car object in the figure with sharp depth transitions around the wheels), which were not accounted for in our study. In these cases, areas with smooth depth (car bonnet) are visually similar, but artifacts at discontinuities are visible even at high depth resolutions.

Type 3 occlusion artifacts are much more noticeable so we suggest that an occlusion mask or layer is created/detected to mask out virtual objects behind the real world. This is easier to create than a mesh, which must also be manually aligned with the background. The mask can remain in the equirectangular domain for simple compositing.

Types 2 and 4 present a more interesting challenge as they are both caused by real 

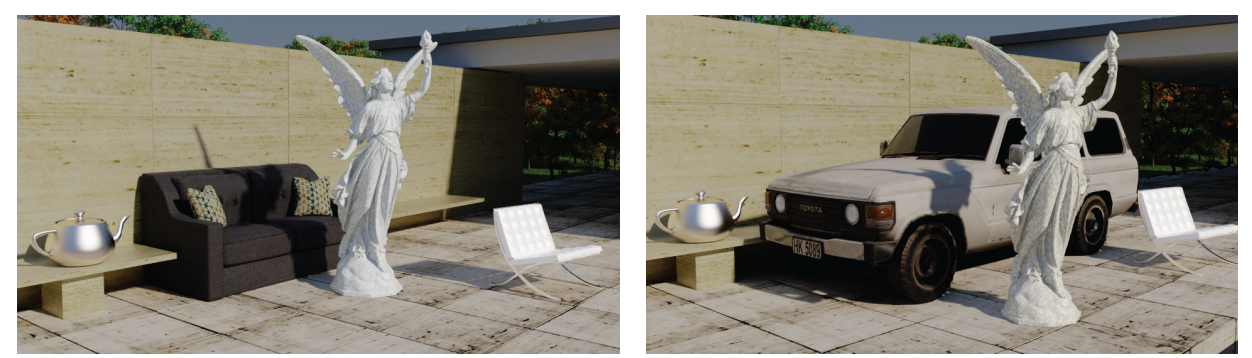

(a) Composite $4096 \times 2048$
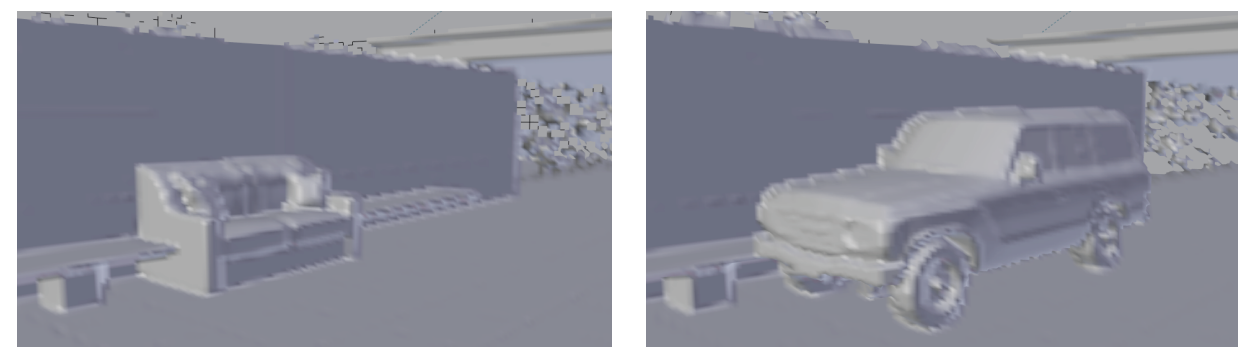

(c) Mesh 4096×2048
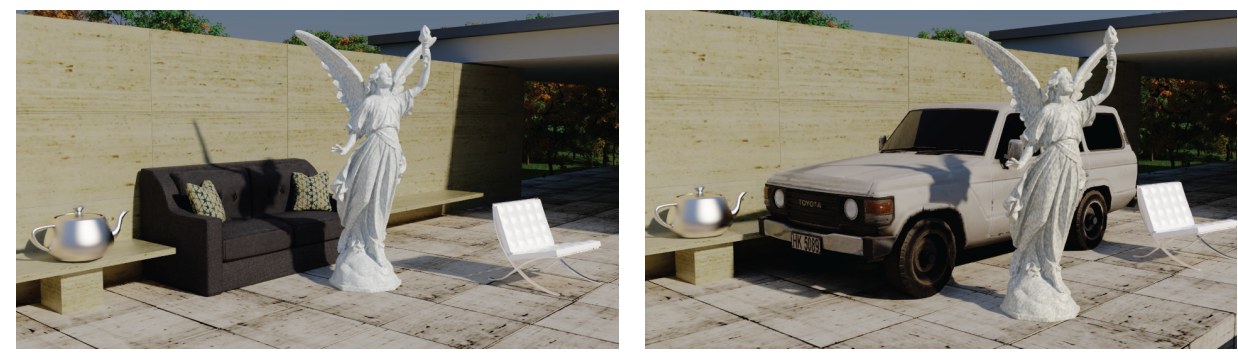

(b) Composite $426 \times 213$
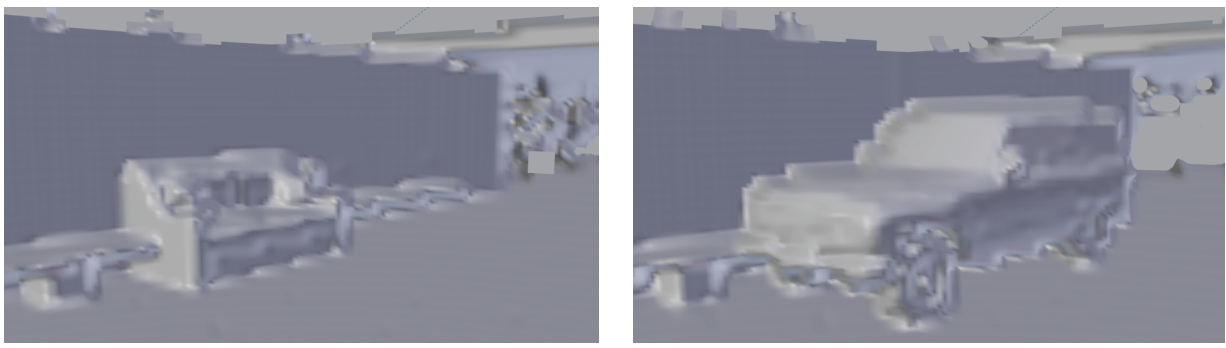

(d) Mesh $426 \times 213$

Figure 2.8: Example application of the Type 1 perceptual threshold in MR compositing into a photorealistic background. The glossy teapot, statue and white chair, are the inserted objects. Compositions with a $4096 \times 2048$ depth map (a) and a perceptually optimal $426 \times 213$ depth map (b). Reconstructed geometry for the composites is also shown: meshes with $4096 \times 2048$ (c) and $426 \times 213$ (d) depth maps. Top row: composition into a background scene with continuous depth (couch object). Bottom row: fail case of composition into discontinuous depth (car object with artifacts around wheels in both (b) and (c)). 
world shadow casters but have vastly different noticeability. The low noticeability of Type 2 indicates that the blocker geometry can be low fidelity, but this will in turn increase the Type 4 artifact severity. Therefore, we suggest creating/detecting a shadow region matte to mask the shadow overlap. This combination trades off optimised geometry fidelity for requiring a shadow matte input. Both steps combined are often less resource intensive than creating or detecting high fidelity geometry to solve both problems. The coarse blocker geometry could be automatically detected via segmentation and stored as layers [66, 67]. Similarly, shadow detection has also been automated in previous research $[68,69]$.

We measured artifact noticeability in relation to depth in a full FOV equirectangular format. If a standard, lower FOV depth map is used then depth resolutions even lower than our perceptual threshold may suffice.

\subsection{Conclusion and Future Work}

The aim of this chapter was to study the required level of fidelity in background geometry for seamless composition. We achieved this by studying the perceptual effects of depth resolution reduction on the noticeability of mutual illumination artifacts. We formalised four artifact definitions and tested these individually. From the results we obtained perceptual thresholds and optimal trade-off points for multiple MR cases. We finally used these findings to demonstrate where resources can be optimised and where more care must be taken.

Disocclusions in depth capture may lead to discontinuous depth, meaning real shadows cannot be fully reconstructed where depth behind occluders is unknown. However, our studies were limited to continuous depth to avoid such artifacts, so further study is needed to evaluate this case. While our studies evaluated artifacts from depth resolution reduction, we assumed the depth was still accurate. Captured or estimated depth may be both inaccurate and low resolution, so depth accuracy limits for perceived seamlessness requires further studies. Our results could be refined with more participants and repeating the study with a focussed resolution range would find finer perceptual thresholds. Having tested on fully 
diffuse scenes, we cannot infer how depth resolution affects perceived reflections on virtual objects from the real world. Measuring perceptual depth fidelity thresholds required for indirect illumination and colour bleeding would also be useful. The experiment setup could be extended to measure how multiple lights and lighting complexity affect seamless shadow composition.

Scenes with objects having large surface variation across small distance (e.g. spiky objects) were not evaluated in our tests. Such high frequency details could be represented with high-resolution depth maps, as long as they do not cause occlusions (creating depth discontinuities) from the depth-camera viewpoint.

Our perceptual thresholds may also fail in dynamic scenes such as those with moving objects and viewpoints Depth resolution artifacts are caused by subsampling the depth signal. The depth of moving objects would be sampled differently every frame, potentially causing temporal flickering. This flickering would be made worse with a moving camera since all points in the image could be changing per frame. Temporal flickering in the depth map could increase artifact noticeability in the region of the shadow being composited. Future perceptual simplification of depth maps for these scenes, including both dynamic and high-frequency objects, could be informed by the Contrast Sensistvity Function $[70,49]$.

Having measured perceptual thresholds in a $360^{\circ}$ depth format, our findings can be applied to new $360^{\circ}$ depth acquisition $[71,72,64]$ techniques which can enhance MR experiences for mutual rendering effects in $360^{\circ}$ content [73]. Further evaluation in dynamic MR scenes would increase the practical use of our results. Composition of animated objects into a video background with dynamic depth could be used in our method. Using animated virtual objects moving in and out of artifact regions could measure if artifacts are more pronounced in dynamic scenes. Further studies using head tracking hardware in a $360^{\circ}$ environment could measure how artifact perception is affected while immersed. It is also worth exploring new depth acquisition algorithms with perceptual optimisation in mind.

This chapter focused on perceptually seamless composition into live action backgrounds. The following chapters focus on art-directing the foreground object. 
The next chapter will present a new workflow for art-directing 3D object appearance. Similarly to this chapter, realistic lighting will be considered while preserving art direction under dynamic lighting environments. 


\section{Chapter 3}

\section{Art-directing Appearance using an Environment Map Latent Space}

\subsection{Introduction}

Look development (look-dev) is the task of verifying that a $3 \mathrm{D}$ model gives acceptable appearance when rendered under different lighting environments [74]. For this task, artists use lighting environments captured from the real world, for high quality pre-visualisation. These lighting environments are captured as high dynamic range (HDR) 360-degree panoramas called environment maps (EMs) [12]. During look-dev, an artist will render a 3D model with its material properties under many EMs with varying types of illumination (e.g. indoor, outdoor-sunny, outdoor-overcast, etc.) to verify adequate shape and material depiction.

This material may give acceptable appearance in some lighting conditions, but not in others. For example, artificial rim lighting may be desired in sunny scenes but not in cloudy scenes. Glossy highlights may need exaggeration in cloudy scenes, but not in sunny scenes. Hence, different EMs call for unique appearance edits, determined by a director's stylistic intentions [25]. Unfortunately, artists currently can only assign a fixed set of material parameters.

To mitigate this problem, manual art direction edits can be made in offline films, but at the cost of artists' time and effort in post-production. However, dynamic 3D 
games and augmented reality applications feature unpredictable and large variation in environment lighting, such that a fixed appearance cannot give satisfactory results under all possible lighting. For these situations, appearance edits need to be pre-defined in pre-production, and then automatically adapted to real-time environment lighting changes during runtime. There currently exists no workflow for artists to specify customised appearance variation across many EMs.

We propose a new workflow to address this problem, using a novel latent space of EM lighting. We learn a 2D latent space from a large and varied database of EMs. Our space visualises semantically meaningful clusters for several types of lighting conditions (indoor, sunny, overcast), in a single 2D plot. The visualisation is firstly used in look-dev, to browse and identify which EM types require appearance editing. Artists can then prescribe the desired appearance edits, unique to different EM clusters in the space. In our interface, artists can paint appearance parameter values for different clusters in the space, and control how parameters transition between the clusters. We then automatically adapt the artistic edits made for each EM to unseen environments in live applications. This workflow is shown in Figure 3.1.

Using the latent space for browsing provides meaningful categorisation of the thousands of EMs used in visual effects studios. Look-dev artists currently verify appearance with a small number of test environments that may not represent lighting variation between those types. Previous EM organisation methods either require manual labelling [36], or infer semantic information [75] irrelevant to lighting properties. Our learned 2D organisation avoids time-consuming probing of higher dimensional spaces when browsing and selecting points using multiple 2D subplots [76, 77, 78]. Unlike these higher dimensional spaces projected into 2D, our space allows per-EM, appearance edits by providing a unique 2D location for all EMs. We needed a highly reduced feature space to ensure that clustering is meaningful. This is not always the case in higher dimensional spaces due to the Curse of dimensionality. in higher dimensions, samples become sparse and equidistant so clusters are difficult to form [79]. We embed in 2D to avoid sparse clusters, so that artists can finely control appearance interpolation between clusters. Many previous art-directable rendering techniques also allow artists to edit 


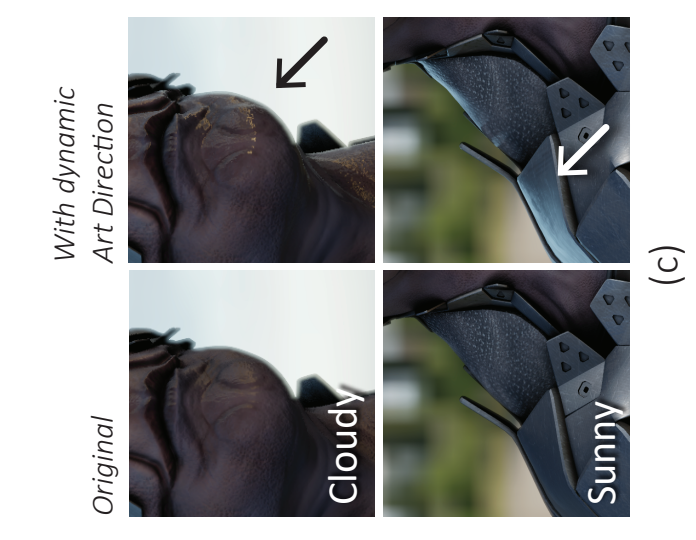

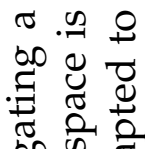

क क त

తิ

范

ลे

तิ

$\exists$ 중

రం

ㅎํㄹ

点.

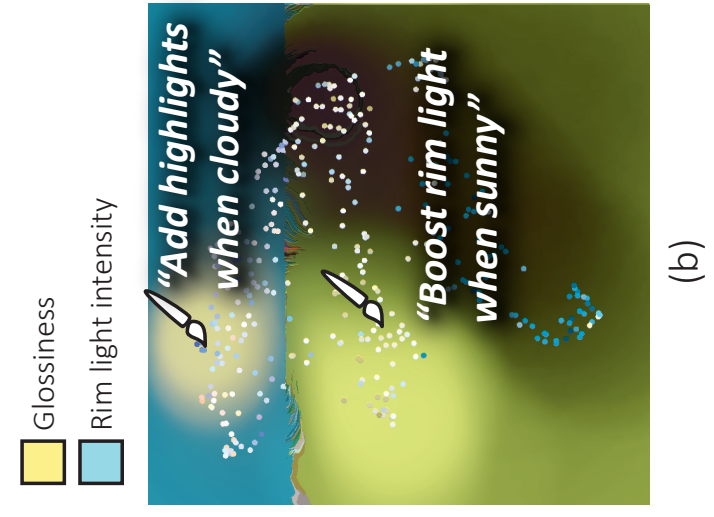

든ㄷㅇ

. 즐

즐 즐.

है क्षे के

๘ $\Xi$

艝

寻

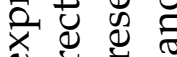

(4)

\& 0 .

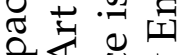

काष्ठ

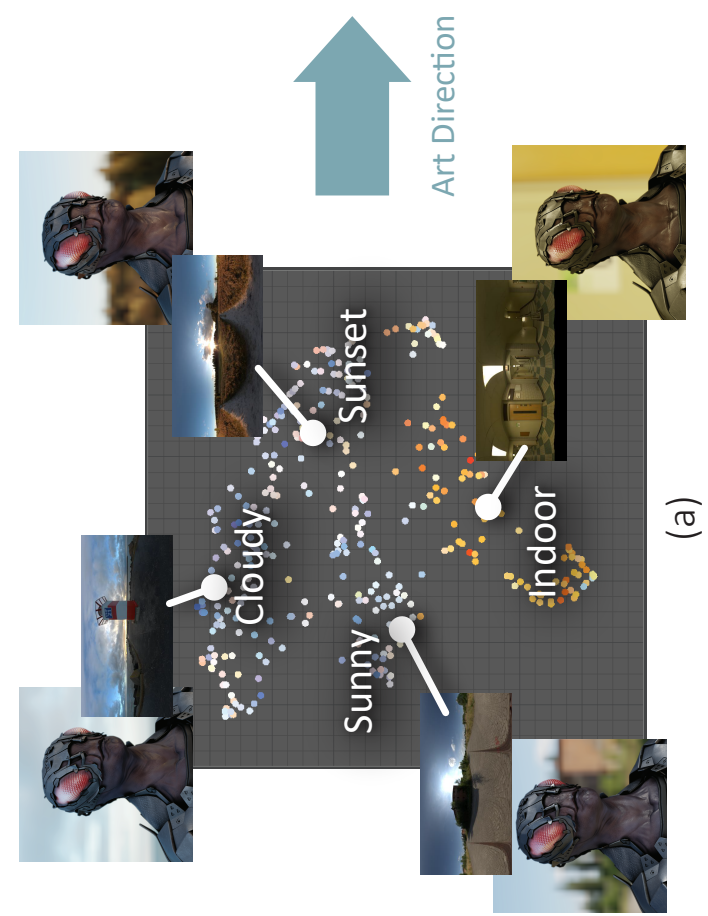

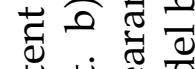

䒕苟 苋

ऽ ह 하

过司实

용

¿્વ ช :

苞䔎选

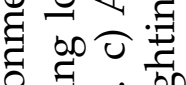

: 000

(0)

के

కี สี สี ปี

○월 응

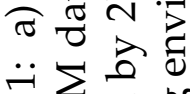

ल

\%

ప. 
appearance, but only in a single scene [27]. Stylisation using illumination guides assumes that lighting is unchanged [26]. Parametric appearance edits can be transferred under slight lighting changes [28], but cannot take unique edits made for different EM types as input. We instead require appearance edits to interpolate between more drastic lighting variation found in large real-world EM databases. Our latent space allows artists to adopt ubiquitous 2D interfaces such as painting to control how shader parameters vary for different lighting types. To preserve the edits, we can consistently encode unseen EMs into the same space, and simply look up the previously painted lighting and material values at the encoded location. We focus on real-world EMs as these are the most common lighting environments used for look-dev. In summary, we make the following contributions:

- We learn a novel 2D latent space with semantically meaningful clusters that express several types of lighting conditions in a single plot.

- We support consistent and robust embedding of unseen EMs to allow edits to automatically adapt to lighting environment changes.

- Our compact EM parameter space allows material and synthetic-lighting edits to transition between environment types. Artists can finely control the transition with painting.

\subsection{Related Work}

Previous work in EM database organisation used manual labelling [36]. While semantically meaningful, this is prone to human error and is impractical for large databases. Texture classification algorithms have been used to categorize panoramas $[75,36]$. This provides encoding of scene content (with labels such as "beach", "church", hotel room", etc). However, these labels lack light source information such as shadow softness, colour, and light direction. More importantly, human-assigned labels with subjective descriptions can be inconsistent. This motivates organising EMs based on mathematical features that retain meaning to artists. 
Several prior works provide techniques to parameterise EMs into low-dimensional representations. Signal processing based compression of illumination such as spherical harmonics [80] and Haar wavelets [81] provide compact descriptions of arbitrary lighting but lack meaning to artists. Conventional abstract light sources (e.g. directional, point, and area lights) found in 3D rendering packages are based on cinematographic lights familiar to artists. However, these emulate simple lighting configurations and cannot represent complex real-world settings without impractical numbers of lights. Mathematical texture features were shown to provide little benefit for lighting tasks [82]. In this work, we use spherical harmonics [80] as a feature for distance measuring between EMs, rather than the descriptor itself. This is similar to previous comparison metrics for $3 \mathrm{D}$ shape analysis [83].

Dimensionality reduction has been relied upon to learn latent spaces and for $2 \mathrm{D}$ data visualisation for many years, and is still relevant today [84, 85, 86, 87]. Similar to our method, 2D latent spaces have been used in artistic applications [88]. Marks et al. [89] introduced the concept of design galleries to aid user parameter selection by embedding high-dimensional outputs into a 2D visualisation. Hermosilla et al. [90] learn a latent space to map 3D scene representations to rendered images. However, we require an artist-interpretable space supporting hand-editable mappings between real-world lighting and art-directed appearance. SkyBrowser [91] provides a four-dimensional feature space to navigate sky-maps in a large database. This is a mathematical yet meaningful EM representation for improved consistency over human-labelled organisation. However, these features focus on textural and colour-based features for sky images. Lalonde et al. use dimensionality reduction for 2D visualisation of learned EM features for low dynamic range (LDR) [92] and HDR outdoor data [93]. However, their HDR dataset embedding is limited to outdoor EMs. Their LDR embedding includes indoor data but does not spatially separate these from outdoor EMs. Our method achieves separation of indoor and outdoor EMs in 2D.

For the problem of art direction during look-dev, there is limited research in remapping appearance based on illumination. Many previous techniques support artist editing of realistic renderings to achieve different aesthetic goals [27]. 
However, these methods still require artists to manually edit appearance whenever lighting changes. X-toon [94] also uses a 2D interface for artists to remap shader parameters with respect to two rendering attributes at a time. We instead encode several semantic EM attributes at once, into 2D clusters, for greater art direction flexibility. The patch-based synthesis work StyLit [26] provides user defined mapping between physically based shading and stylisation. Users can define how physically based lighting effects are reproduced in a non-photorealistic manner. However, examples can only be given for one lighting environment. If the lighting in the target image is changed, the highlights and reflected colours also change, causing artist directives to no longer be adhered to. The parametric appearance edits of Hennessey et al. [28] adapt to lighting variation. However, users cannot specify the types of EMs in which edits should be applied.

\subsection{Method}

We describe our method starting with learning the latent space, by embedding a training dataset of EMs in 2D. We then describe our interface for art-directing appearance for different EM clusters, and our method to preserve art direction in new EMs.

\subsubsection{Data Preparation}

We collate existing indoor and outdoor EM datasets from Dutchskies [95], HDRIHub [96], HDR-Maps [97], and Weber et al. [82]. We arrive at a training dataset of 350 EMs after balancing the number of available indoor and outdoor samples. We first horizontally rotate all EMs to center-align them by their dominant light (DL) azimuthal angle. We detect the DL direction by filtering noise and detecting the peak intensity for each EM and align them based on their DL's azimuthal angle. This separates the DL azimuth angle as an independent variable from the latent space. Two EMs with similar lighting but different horizontal rotation, are thus considered to be similar. Horizontal rotation can be independently adjusted in the 
rendering software as necessary. We do not align the DL elevation as it is often correlated with sky colour and lighting softness due to atmospheric scattering, in outdoor environments. Similarly for indoor EMs, artificial ceiling lights and natural light from windows are typically found. Since aligning the DL elevation would tilt the horizon, it can also give unnatural lighting.

\subsubsection{Learning the Latent Space}

We use the recent manifold learning method UMAP [87] to reduce the dimensionality of the training dataset into a 2D space. UMAP is the current state-of-the-art manifold learning technique for 2D visualisation. While CNNs can learn high quality high-dimensional features, UMAP outperforms other methods specifically for $2 \mathrm{D}$ visualisation. For the resultant $2 \mathrm{D}$ space to have semantically meaningful axes and clusters, UMAP requires a distance function that can compute the similarity between two EMs. We define this distance function using a combination of the EM's spherical harmonics (SH) coefficients [98], and its mean colour in LAB space [99].

Rather than using all pixels in the EM, we use $\mathrm{SH}$ to remove texture detail. We compute $\mathrm{SH}$ coefficients up to the $5^{\text {th }}$ band, enough to capture high-frequency details to differentiate between hard, soft, and multiple lighting. We compute this for each of the red, green and blue channels in the EM resulting in 75 features from SH decomposition for each EM ( 25 coefficients up to the $5^{\text {th }}$ band $\times 3$ channels).

Although we compute SH for each colour channel, we would like the distance function to differentiate colours in the EM as a human would perceive them. Hence, we use the mean colour (or first SH band coefficients) of the EM in LAB space as an additional feature. We divide the $\mathrm{SH}$ coefficients by their total $\ell_{2}$ norm to normalise intensity variation in the EMs, and divide the LAB values by 100 (maximum luminance value) for comparable ranges between $\mathrm{SH}$ and $\mathrm{LAB}$. The Euclidean distance between the concatenated $\mathrm{SH}$ and LAB feature vectors is used as the distance metric to compare EMs in the UMAP embedding.

We then need to choose how important we consider the SH coefficients over mean 


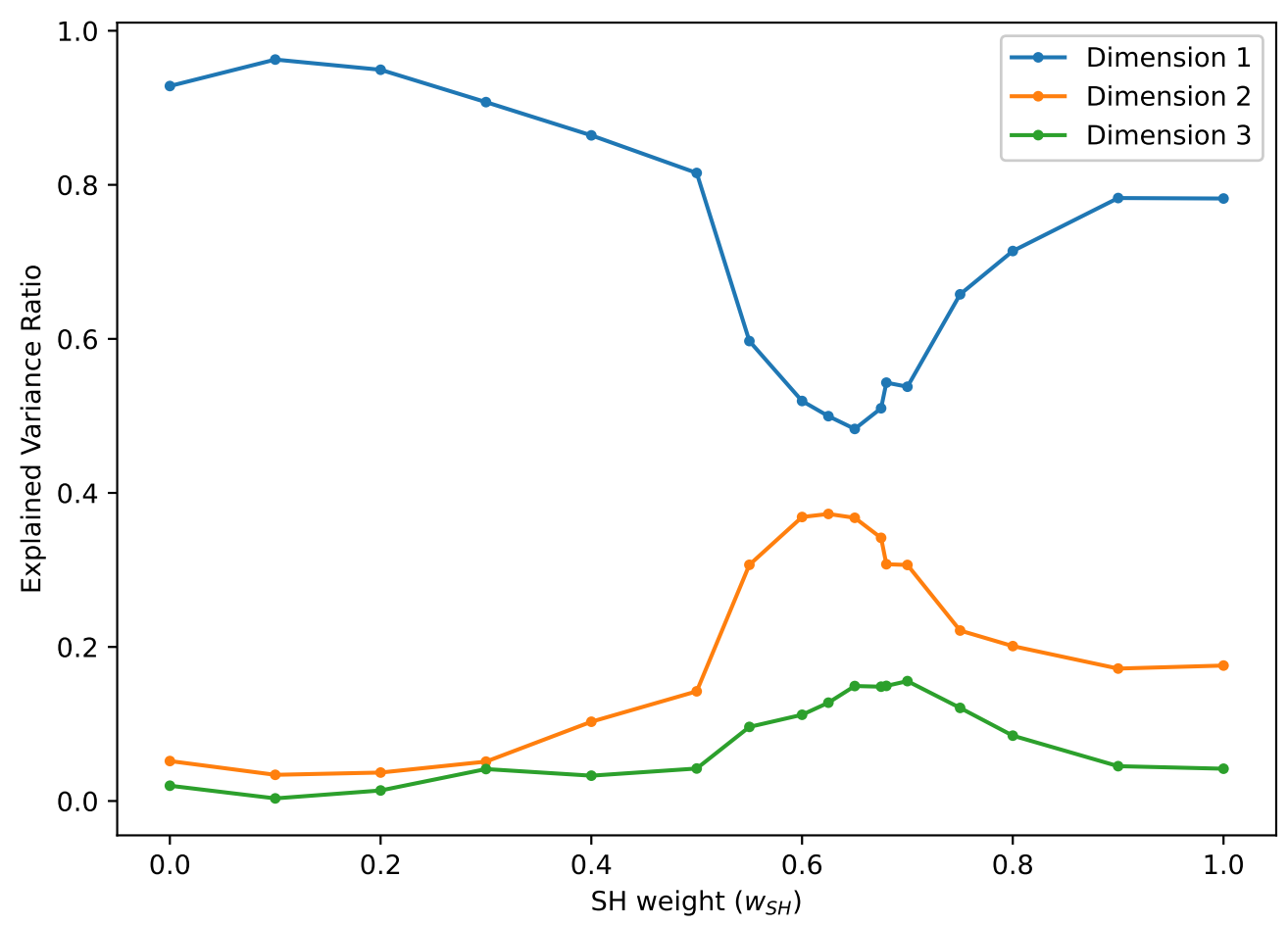

Figure 3.2: Explained variance ratio from PCA dimensions of 3D embedding, for different $w_{S H}$. Between 0.6 and 0.7 , variance is almost evenly shared across two dimensions for our training set.

LAB colour using a weight $w_{S H}$. For example, if LAB is given a large weight over $\mathrm{SH}$, then EMs will primarily be compared by their average colour rather than directional lighting properties. This would result in the embedding to lack separation of varying lighting properties. Furthermore, the LAB feature itself already provides a low-dimensional space, being only three values. To determine the value of $w_{S H}$ we observe how it affects embedding.

We first embed the training dataset in 3D and check the variance in each dimension using PCA. In Figure 3.2 we plot the explained variance of each dimension from PCA, as the weight $w_{S H}$ varies between 0.0 to 1.0. We see that between 0.6 and 0.7 , the explained variance comes close to being evenly distributed along the first two dimensions. This shows that this LAB weight value gives maximised variation 
across two dimensions. At other values, the variance is mostly distributed along one dimension, thus embedding in 3D or even 1D would fail to express the semantic categories we observe in the $2 \mathrm{D}$ result.

Empirically we see that the LAB feature helps to increase the distance between indoor and outdoor environment maps (EMs) in the 2D feature space. In Figure 3.3 we show that increasing the influence of $\mathrm{LAB}$ over $\mathrm{SH}$ features helps distance indoor EMs from outdoor EMs, which reveals clear clustering. Hence, we set $w_{S H}$ to 0.68 clusters the indoor EMs separately while minimally biasing the embedding towards a purely colour-based arrangement.

\subsubsection{Art Directing Appearance Using the Latent Space}

Having learned the 2D organisation of EMs, we use a 2D painting interface for artists to vary appearance parameters, of their choice, across the space. In our interface, we map an image texture for each parameter to our space, such that it spans all points in the embedding. We overlay the embedding, on top of the texture. Artists can then paint the desired appearance parameter values for different EM clusters, directly on the space. Artists can choose to art-direct any parameter in the 3D rendering application (including material, lighting, colour grading, and compositing parameters). They can paint greyscale intensity values for scalar parameters, or with colour for RGB colour parameters.

To preserve edits under changing lighting, we leverage the encoder that UMAP simultaneously learns with the embedding. We use this encoder to embed new EMs, unseen during training, into the same learned clusters. To preserve art-directed appearance in a new EM, we compute its SH and LAB features (as in Section 3.3.2) and encode them into the latent space. We use the new EM's location in the space to look-up the scalar intensity values painted in the texture. We apply the sampled parameter values in the rendering. We show an example of this application in the next section, after examining the latent space. 


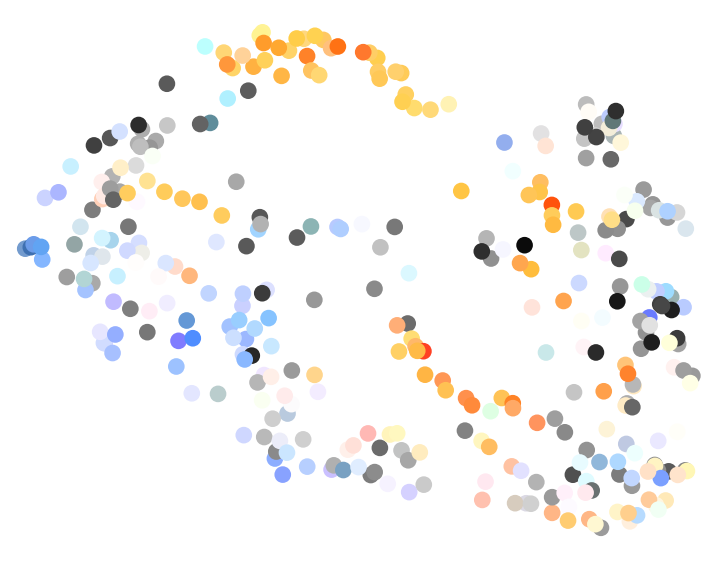

a) $\mathrm{w}_{\mathrm{SH}}=1.0$

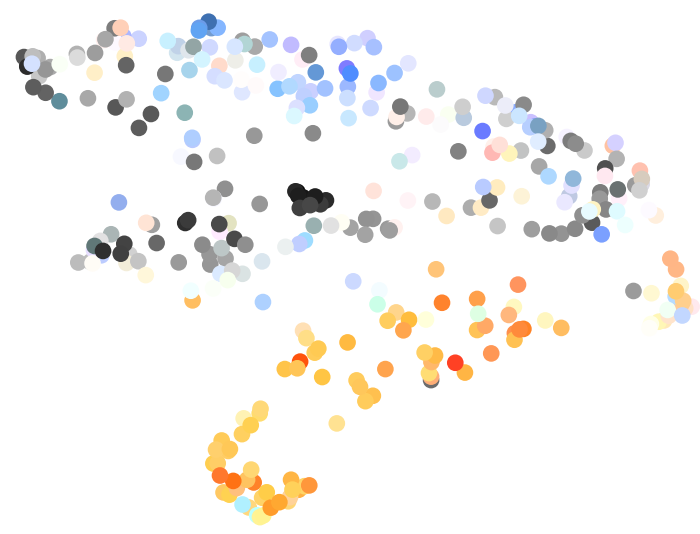

c) $\mathrm{w}_{\mathrm{SH}}=0.68$

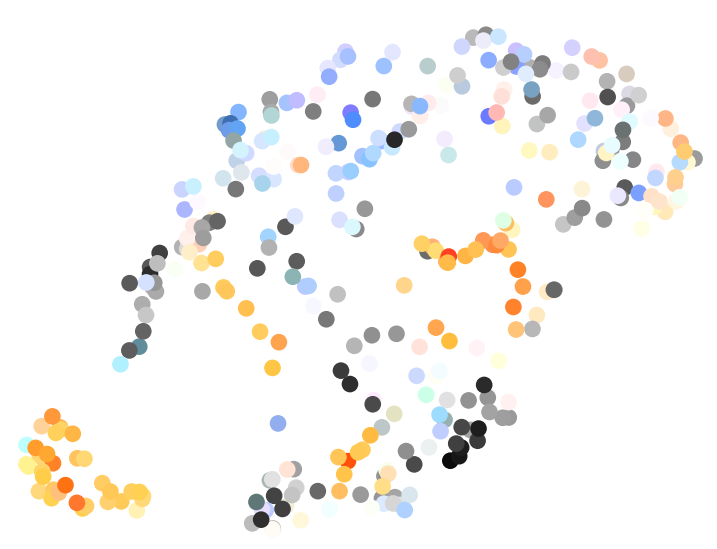

b) $\mathrm{w}_{\mathrm{SH}}=0.8$

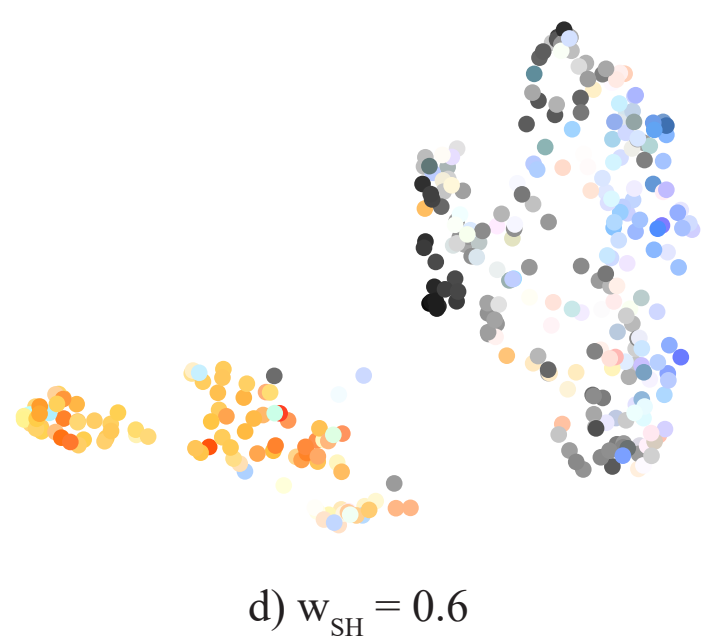

Figure 3.3: a) Using only SH $\left(w_{S H}=0\right)$, indoor EMs (mostly warm colours) and outdoor EMs (mostly cool colours) are embedded too close together. b) Weighting $\mathrm{SH}$ by 0.8 leads to a slight distance increase, but not meaningful clustering. c) Weighting $\mathrm{SH}$ by 0.68 successfully forms a distinct indoor EM cluster, while preserving sunny and overcast clusters. d) If $w_{S H}$ is too high, the embedding is biased toward colour and devalues lighting properties. 


\subsection{Results}

After embedding the training set with respect to our $\mathrm{SH}$ and LAB colour distance metric, we examine the learned latent space for semantically meaningful organisation. Figure 3.4 gives an overview of the observed spatial organisation expressed by the embedding.

We firstly observe that our embedding organises EMs by their light elevation along the $x$-axis. The elevation of the dominant light in the environment decreases as the $\mathrm{x}$-axis increases. This places sunset skies on the right of the space, as the elevation of the sun is low. Similarly, indoor scenes are also consistently organised by the elevation of their dominant light source in the scene.

Secondly, we observe clustering of EMs into three semantic categories outdoor overcast, outdoor sunny, and indoor. These clusters are spread across the y-axis, while maintaining the same ordering of light elevation across the x-axis. Outdoor overcast EMs that cast uniformly soft shadows are concentrated at the top region of the space. Outdoor sunny EMs with hard directional shadows are typically found in the middle. The indoor EMs are found below the sunny cluster. These environments typically have multiple artificial lights, or a mixture of artificial and natural lighting from windows.

\subsubsection{Observed Semantic Clustering}

Figures 3.5, 3.6, and 3.7, examine variation within the clusters of each lighting category. Within the overcast (Figure 3.5) and sunny (Figure 3.6) clusters, we see EMs transition to sunset/sunrise from left to right in the space. Figure 3.7 shows meaningful organisation of EMs within the indoor cluster. Indoor environments with artificial light are concentrated toward the left of the cluster. Transitioning toward the right, we see more EMs with natural light from windows.

Since EMs are organised by light elevation, this transition is expected as windows are typically found on walls and thus have lower elevation than artificial ceiling lights. Due to the global ordering of EMs with light elevation, we see rooms with 

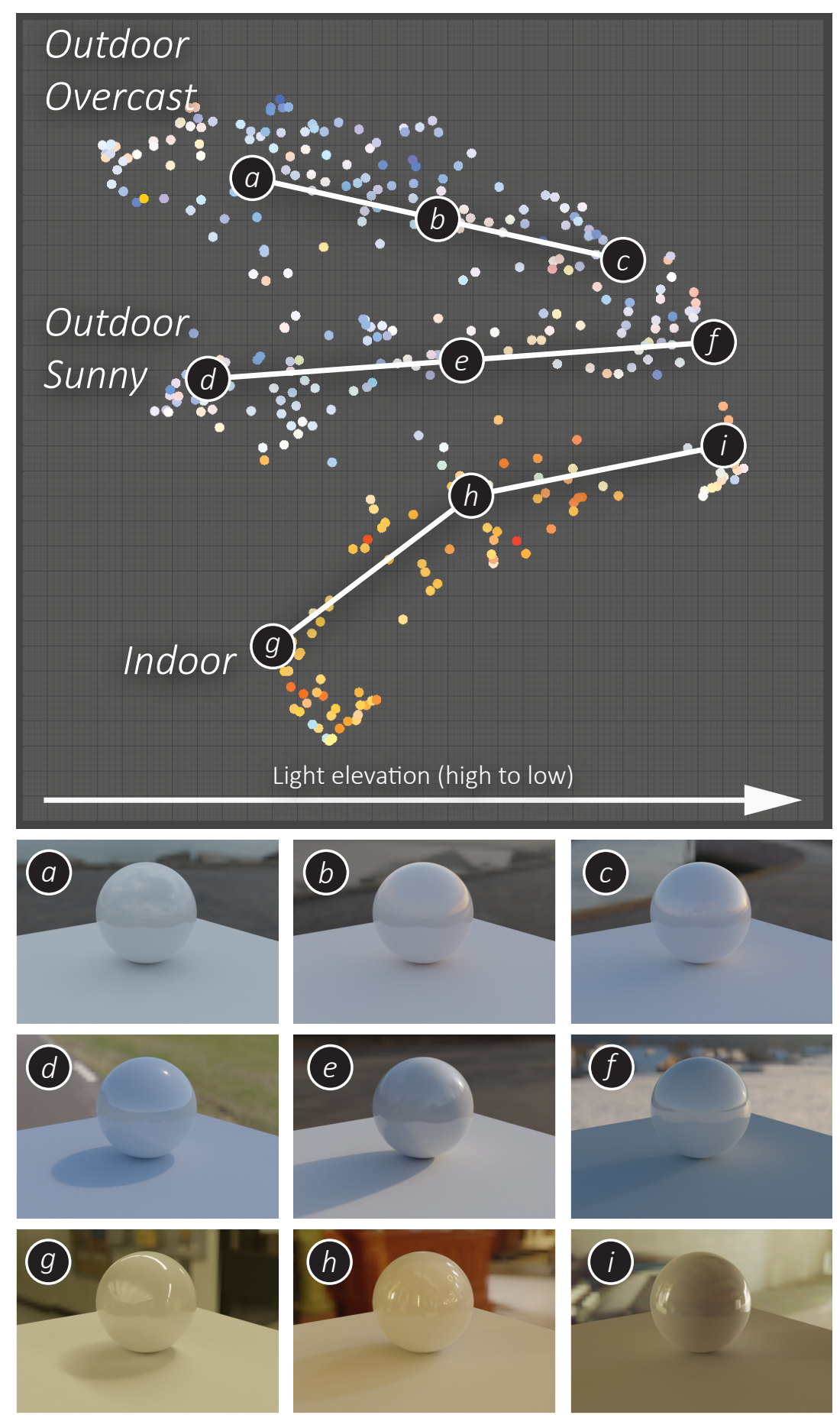

Figure 3.4: Latent space EMs plotted as points coloured using their mean RGB colour. We observe overcast, sunny, and indoor EM clusters shown by soft, hard, and multiple shadows, respectively, rendered below. Light elevation (seen in highlight positions and shadow length) varies consistently across the clusters in the x-axis. 

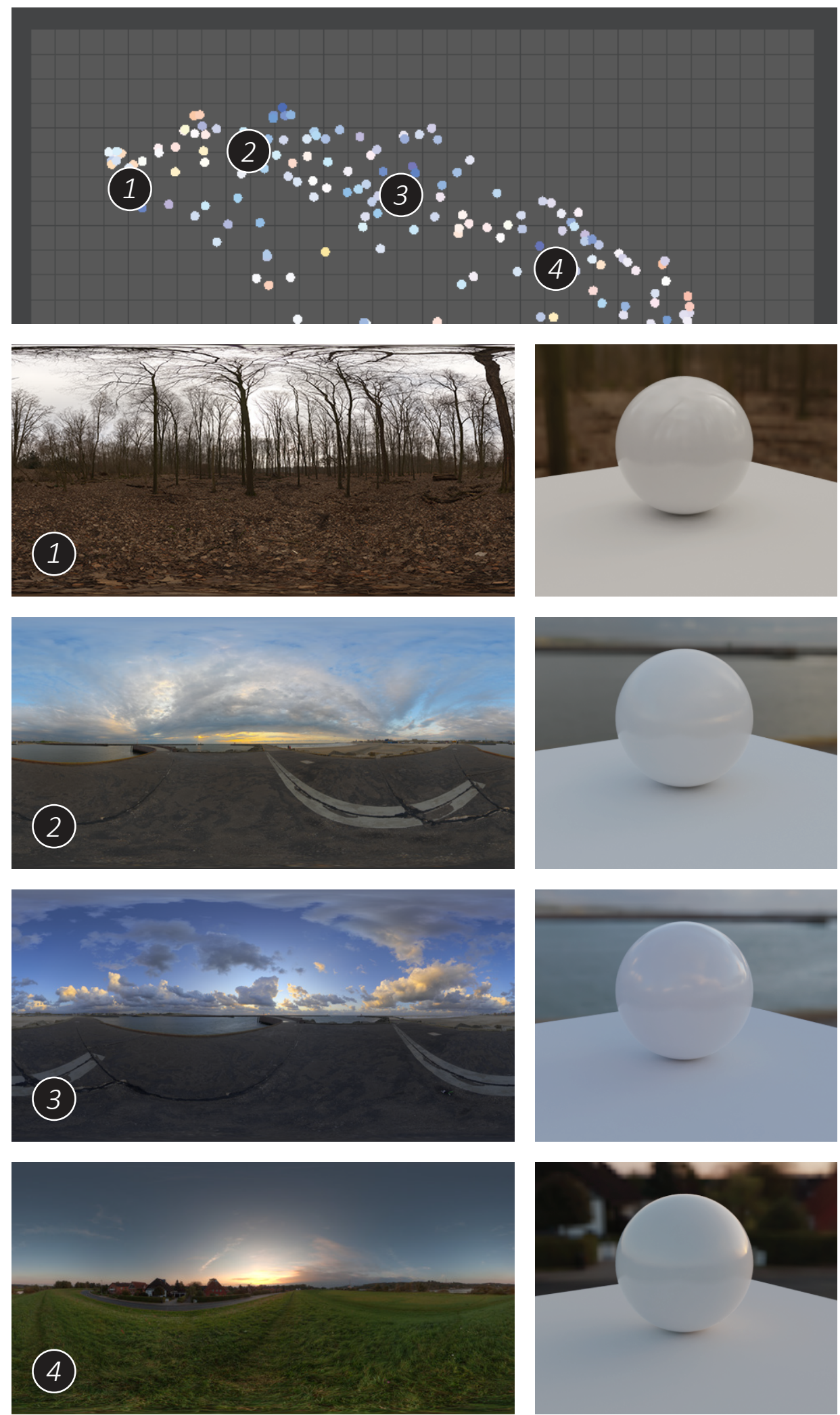

Figure 3.5: Samples found in the observed outdoor overcast cluster of our latent space. 

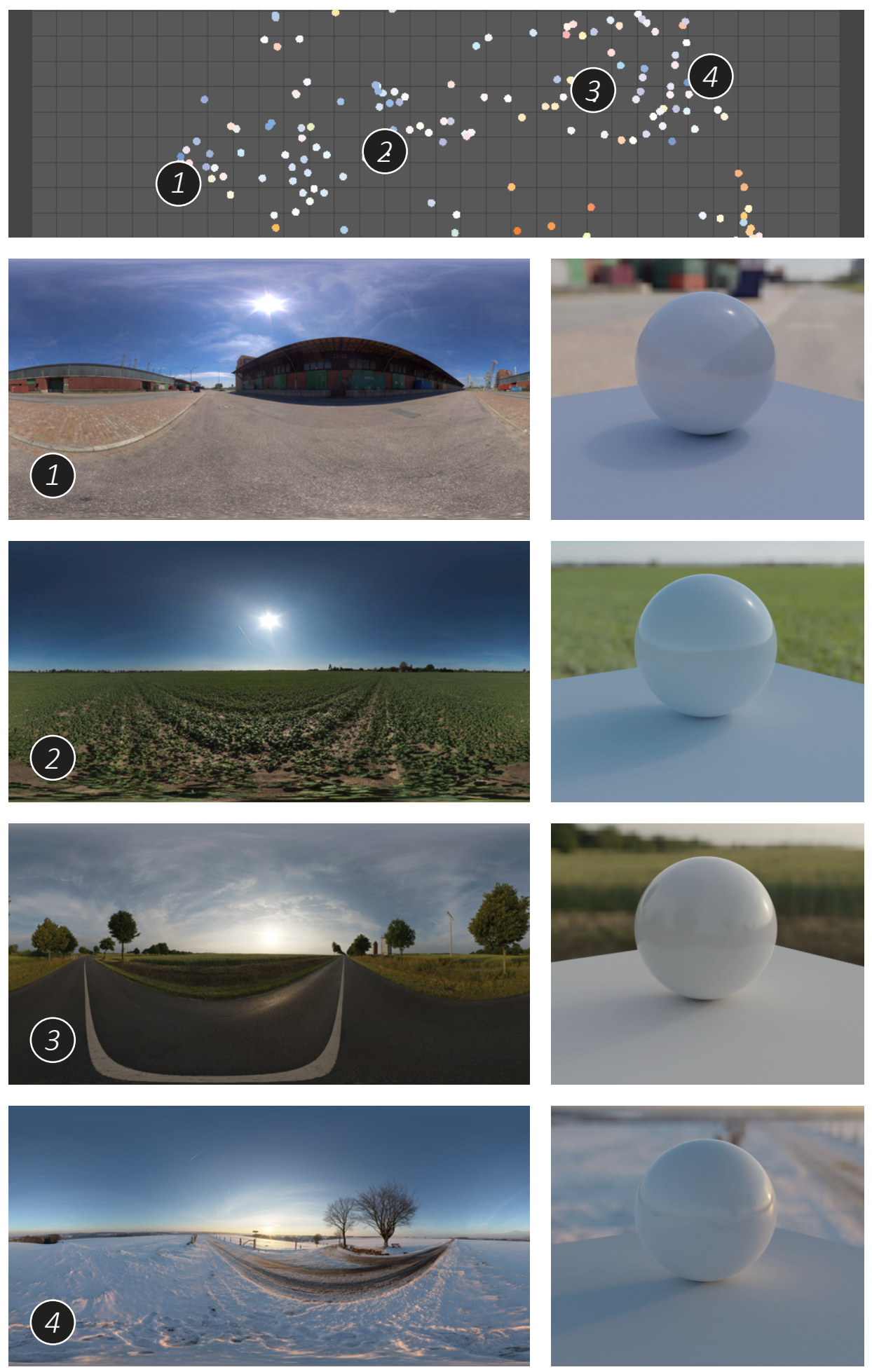

Figure 3.6: Samples in the observed outdoor sunny cluster. 

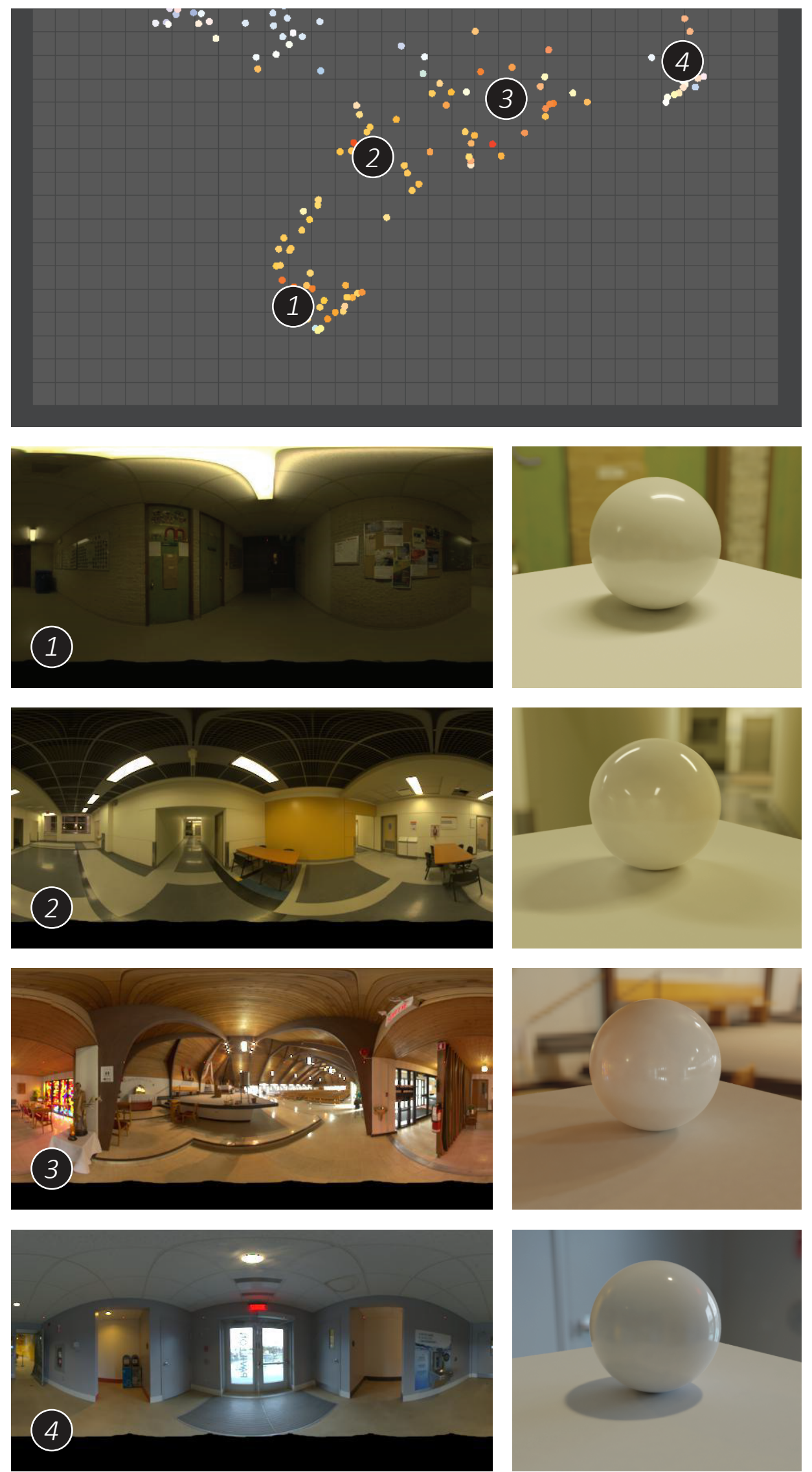

Figure 3.7: Samples in the observed indoor cluster. 
sky light windows on the ceiling are embedded into the high-elevation region rather than be clustered near the low-elevation window scenes. Similarly, indoor EMs with large windows are often clustered in the sunny or overcast clusters, due to their lighting properties being more similar to outdoor EMs than those in the indoor cluster.

We note that it is still unclear whether users would think all natural lighting should be in the same cluster, or if they should have a more high-level organisation based on light elevation. This depends on subjective notions of what categories of EMs are semantically similar. This research instead focuses on learning a space that is consistent in its semantic organisation, and remains the first to achieve this in a single 2D plot.

We also show that our space can express lighting conditions considered to be between clusters. Between the Overcast and Sunny clusters, we see a transition from soft to hard shadows. In Figure 3.8 we observe that points near the top of the overcast cluster are scenes with heavy cloud coverage, casting soft shadows. Transitioning further down to the Sunny cluster, we see more scenes with partial cloud cover giving slightly sharper shadows. Then, close to the sunny cluster, we observe scenes with sharper shadows caused by partial tree or building coverage.

In figure 3.9 we see some points between the sunny and indoor represent a transition from sunny outdoors to indoor with varying window size, then indoor with only artificial light. However, these areas between clusters are sparse. This suggests we have insufficient data for these environment types to confirm this behaviour would be consistent with new data.

\subsubsection{Evaluation}

We have shown that the latent space has clearly distinguished semantic clusters. We also show that our space can reliably embed new points into the previously observed semantic clusters. We validate both properties of our space by embedding a human-labelled test set of EMs unseen during training. Using human-given labels we can validate that EMs are embedded into semantically 

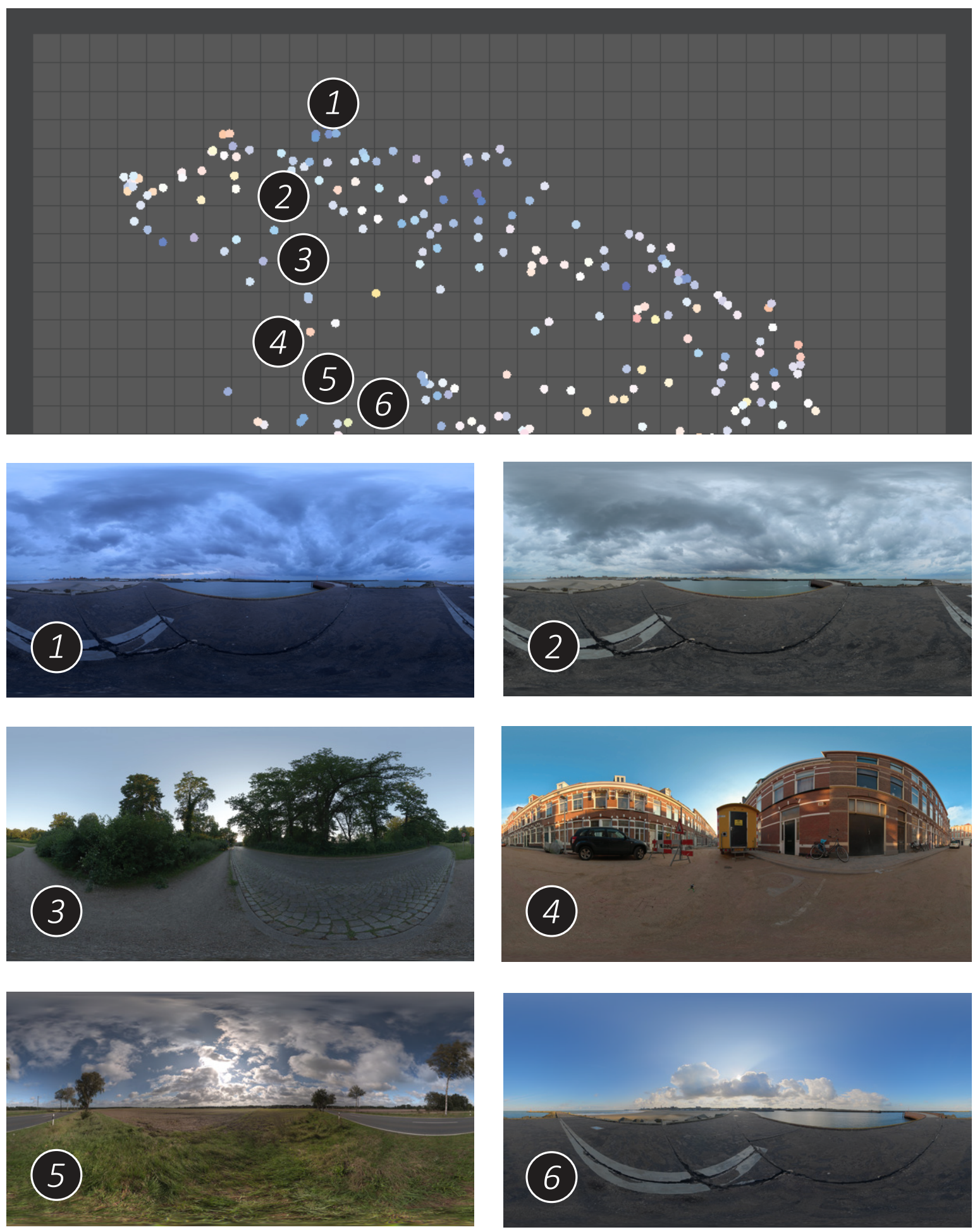

Figure 3.8: Samples between overcast and sunny clusters. From 1-6, we see a transition from heavy cloud, to clear sky with sun occluded, to partial sun occlusion (almost sunny). 

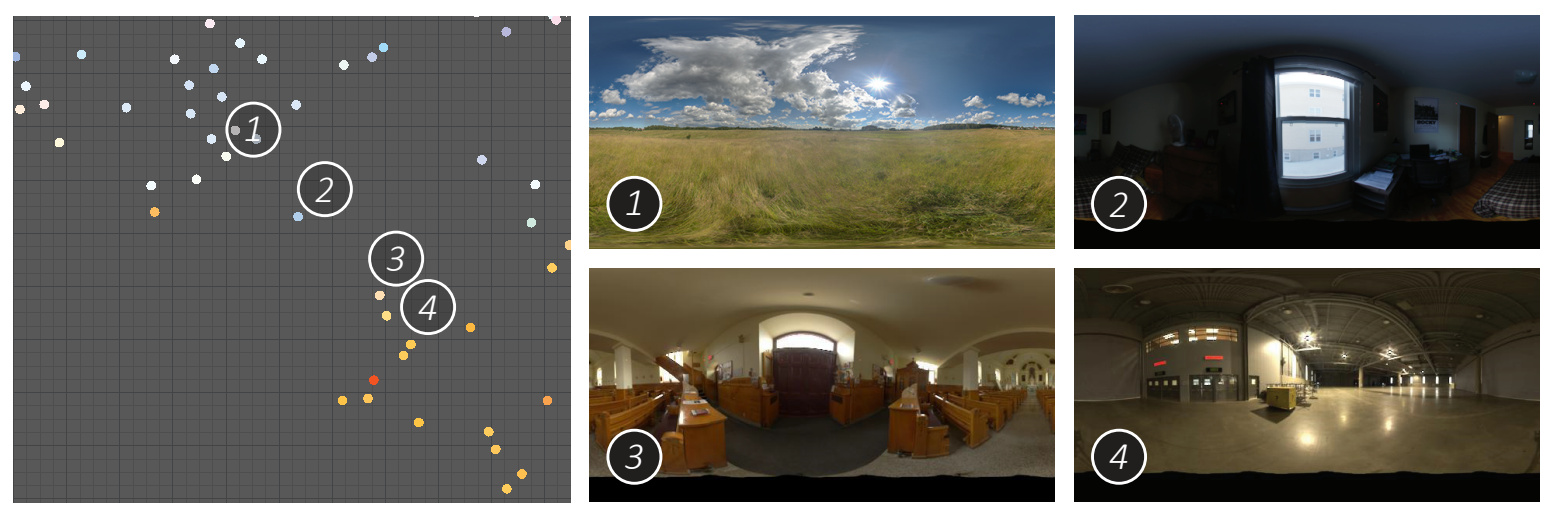

Figure 3.9: Samples between sunny and indoor clusters. From 1-4, we see a transition from fully outdoor and sunny, to large window lighting, to small window lighting, to artificial lighting.

meaningful clusters (more so than simple ordering based on light elevation). Using EMs not originally included in the training simultaneously verifies that unseen EMs are embedded into the appropriate clusters.

Although our space expresses some examples of nuanced semantic clustering (e.g. indoor with natural light, or separating clear and cloudy overcast skies) we evaluate the expression of high-level clusters that are not as ambiguous. We clarify these high-level observed clusters under the following definitions.

Overcast: Outdoor environments casting soft shadows due to the sun being covered by clouds, buildings, trees, or set beneath the horizon.

Sunny: Outdoor environments casting hard shadows, and focused highlights on objects, from unoccluded sunlight.

Indoor-artificial: Indoor environments with dominant lighting from artificial lights.

Indoor-natural: Indoor environments with dominant lighting from natural outdoor lighting from windows.

Night: Outdoor night-time environments lit by street lights, stars, or the moon. We note that these are under-represented in our training set, so no clear night cluster was observed in the original embedding.

We also make distinctions between environments that do not clearly fall within these categories. Indoor environments with mixed artificial and natural, with no 
clear dominant lighting type, are considered ambiguous (e.g. room with both a large window and bright ceiling light). Outdoor EMs with poorly captured dynamic range (incorrectly representing the brightness of the sun) are also excluded as they may be sunny but cast soft shadows.

Excluding these ambiguous cases, we proceed assuming that our definitions for outdoor sunny, outdoor overcast, and indoor categories are indisputable by humans. Hence, we evaluate using the labels given by the EM vendors. We manually label all unlabelled EMs and verify that all existing labels conform to our definitions.

We collate a test set of 262 outdoor and 80 indoor EMs (after discarding ambiguous cases) from the same sources as the training set, but not used during training. Unseen EMs are encoded by extracting the same spherical harmonics and LAB mean colour features of each EM, as explained in Section 3.3. We encode the EM features with the transform procedure of UMAP (without retraining) and examine the new EM positions in the latent space. In Figure 3.10 we see that the same observed clusters are expressed in the test set embedding. Although night scenes were under-represented in both test and training sets, we see they appropriately appear in at least one outdoor cluster. The overlap between clusters is attributed to noise in the encoding process due the stochastic nature of UMAP. We see only a few points located in the wrong clusters which we consider outliers. This is a limitation of our method as we do not enforce the learning of assumed labels during training. We also test the quality of embedding samples from the HDRI-Haven outdoor dataset [1] (156 samples after filtering). We see that these are embedded in the expected location, even though our training set did not contain any samples from the HDRI-Haven vendor. In Figure 3.11 we see the same expected clustering behaviour, showing our encoder is robust to outdoor environments with capturing processes different to those found in our training set. We also see clearer semantic clustering seen in Figure 3.10 such as the much narrower sunny cluster. This could be because the EMs from this vendor have much greater dynamic range than those used during training. This perhaps makes them easier for UMAP to differentiate based on our distance metric. Within the overcast cluster of this embedding, we find EMs with full cloud or building cover of the sun, located at the top. Then we 


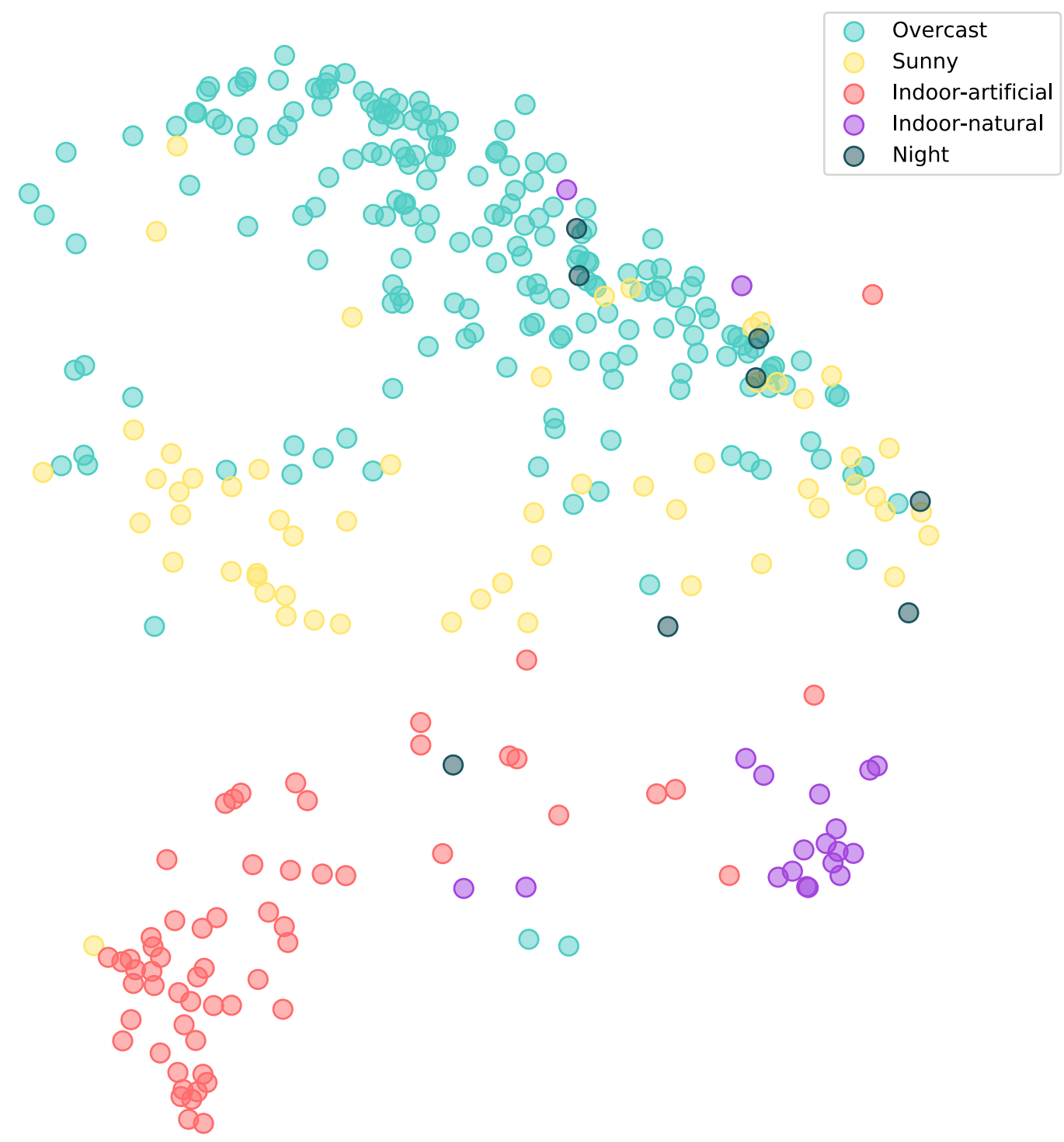

Figure 3.10: Encoding a test set of unseen EMs into our latent space. The new samples are concentrated in the same clusters observed in the original embedding. 


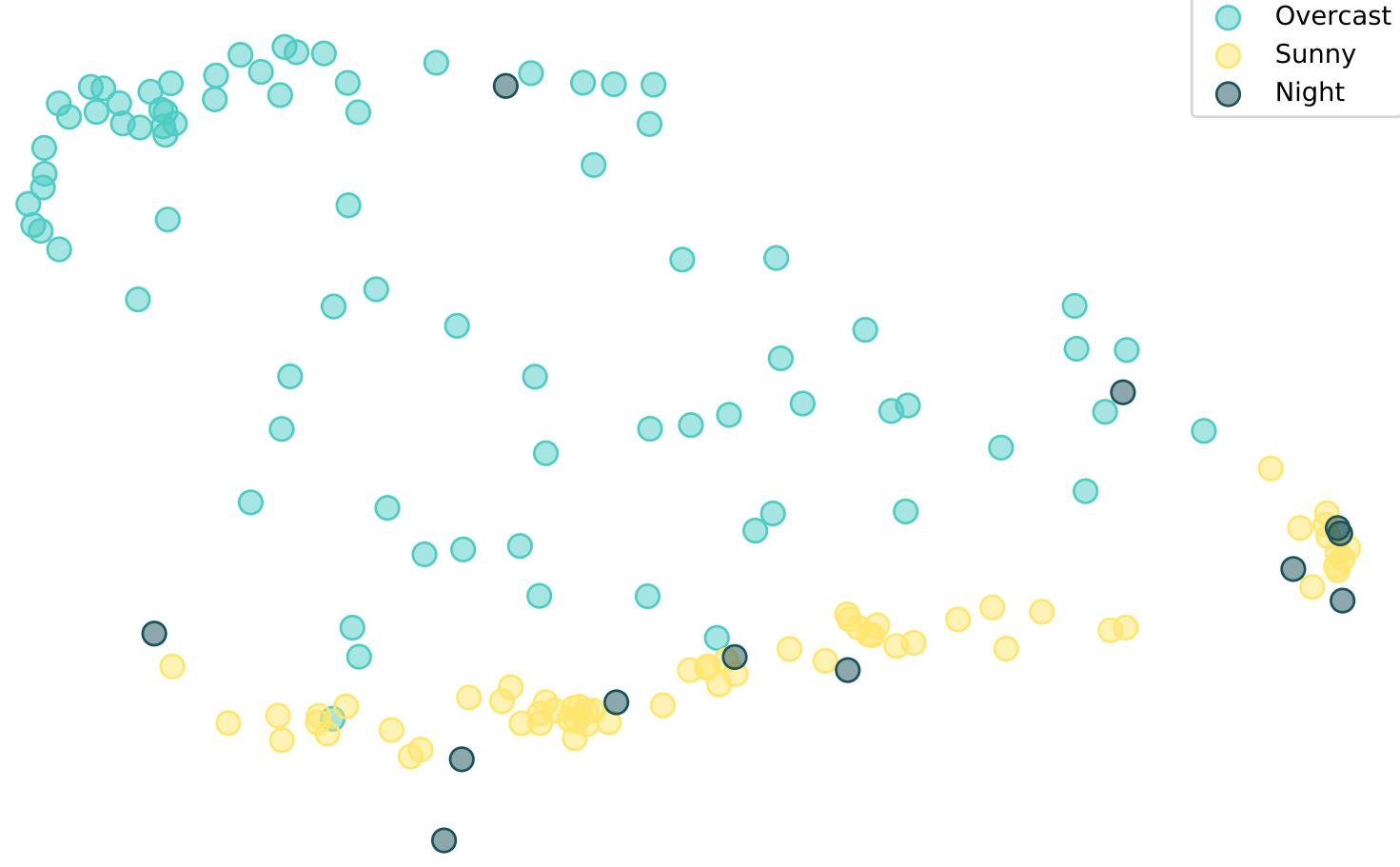

Figure 3.11: Encoding unseen EMs from the HDRI-Haven outdoor dataset [1]. The new samples are concentrated in the same clusters observed in the original embedding. 
see a transition to EMs with partial cloud or tree cover of the sun when moving down toward the sunny cluster. Like the first test set, outdoor night scenes are also encoded into at least one outdoor cluster. Only two outliers are found in the indoor cluster. It took between 1 and 2 seconds to encode the test datasets into the latent space. This time was measured on a laptop with an i7-4700MQ 2.4GHz CPU, with 16GB of RAM.

\subsubsection{Art direction Application}

We apply our latent space as an appearance control space for use during look-dev. We tested with an industry look-dev use-case from Blender Animation Studios. The example character in Figure 3.12, was lit under several different lighting environments to verify consistent appearance. Highlights on the skin became far less apparent in the overcast EMs, compared to sunny and indoor EMs. In this situation, the artist could increase the skin material's glossiness to restore highlights. Doing this, however, causes overly glossy appearance in sunny and indoor scenes. Using our latent space, artists can find which kind of EMs give problematic appearance. By simply painting intensity values in the latent space, artists can indicate under what lighting conditions should the glossiness be increased, and by how much. The result is the desired behaviour of highlights being enhanced in cloudy scenes, and unaffected in others. Having shown the latent space reliably encodes unseen EMs, such art-directives will be automatically adhered to in similar future lighting scenarios. This removes the need for repeated appearance edits and automatically preserves art direction in games, and live AR applications. In figure 3.13 we show an example appearance transition between EM types, painted using our interface.

We also apply our art direction method to highly stylised cartoon shading in realworld composites, as shown in Figure 3.14. Here artists have full control of how toon shader parameters respond to real-world lighting. In this example, outlines were hidden in sunny scenes, and shading was softened in overcast and indoor scenes. Such high appearance variation can be achieved with our method. 

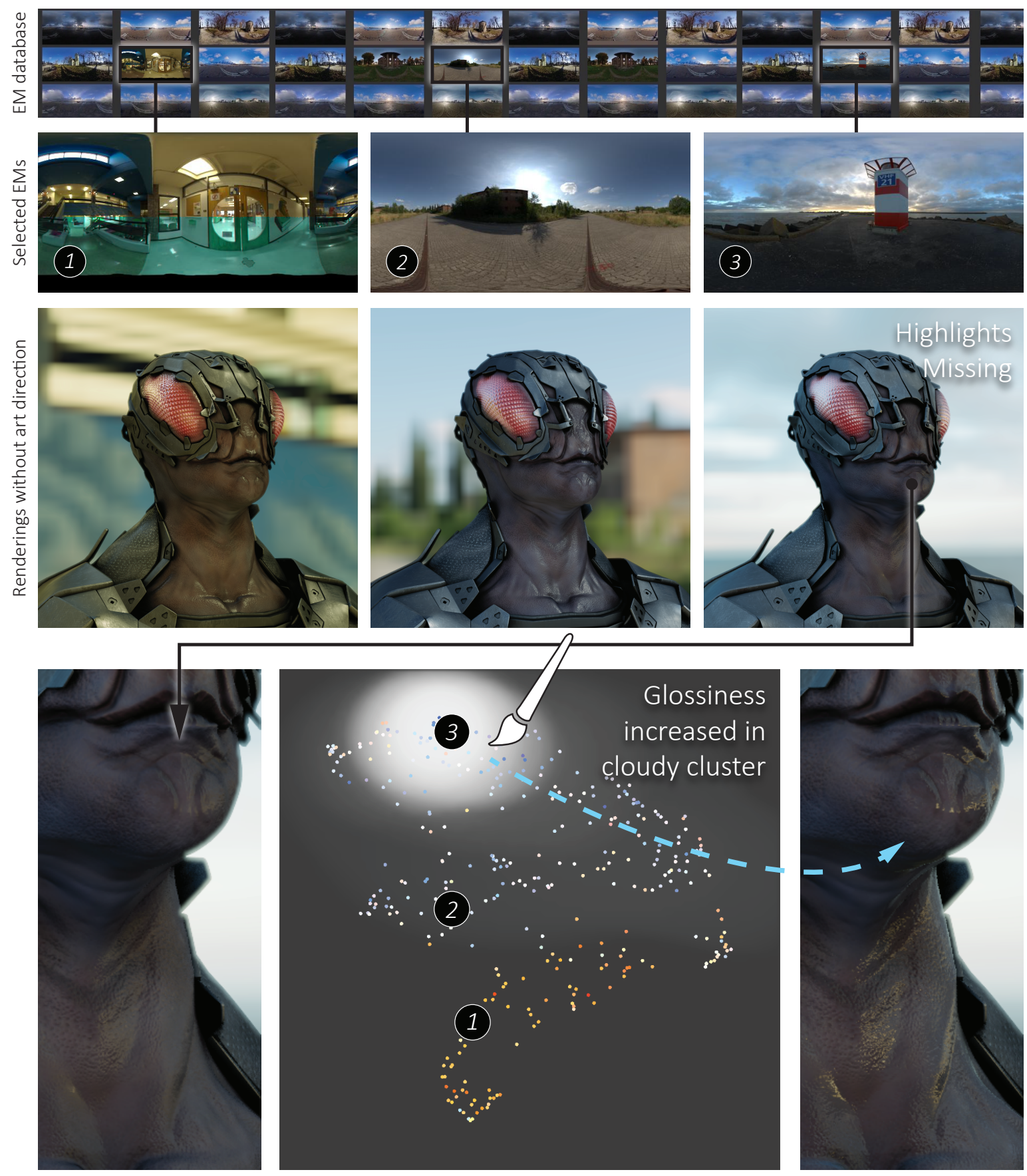

No art direction

Painted map of parameter values

Art-directed

Figure 3.12: Application of the latent space as an appearance control space. Top: Lighting environments used in look-dev, with 3D character preview under the same lighting below. In the third preview, highlights are lacking. Bottom: latent space is used to specify increased glossiness, by painting brighter intensity values in the region of cloudy EMs. This enhances highlights only in cloudy scenes which avoids overexposing them in sunny and outdoor scenes. 

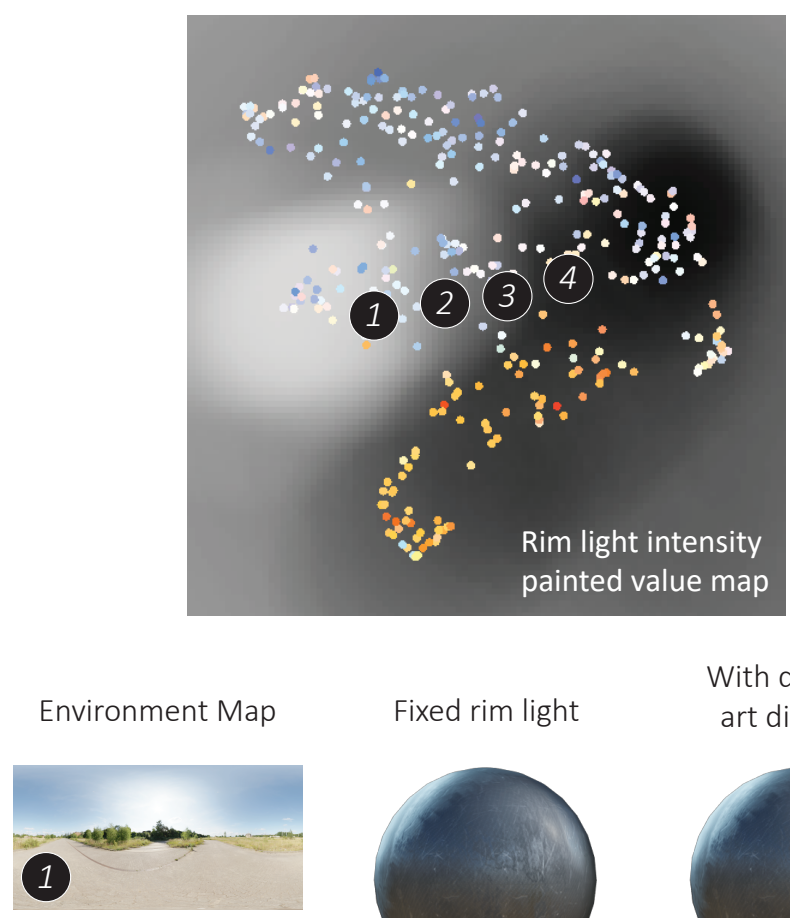

Fixed rim light

With dynamic

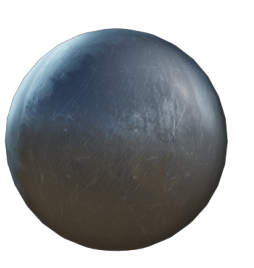

art direction
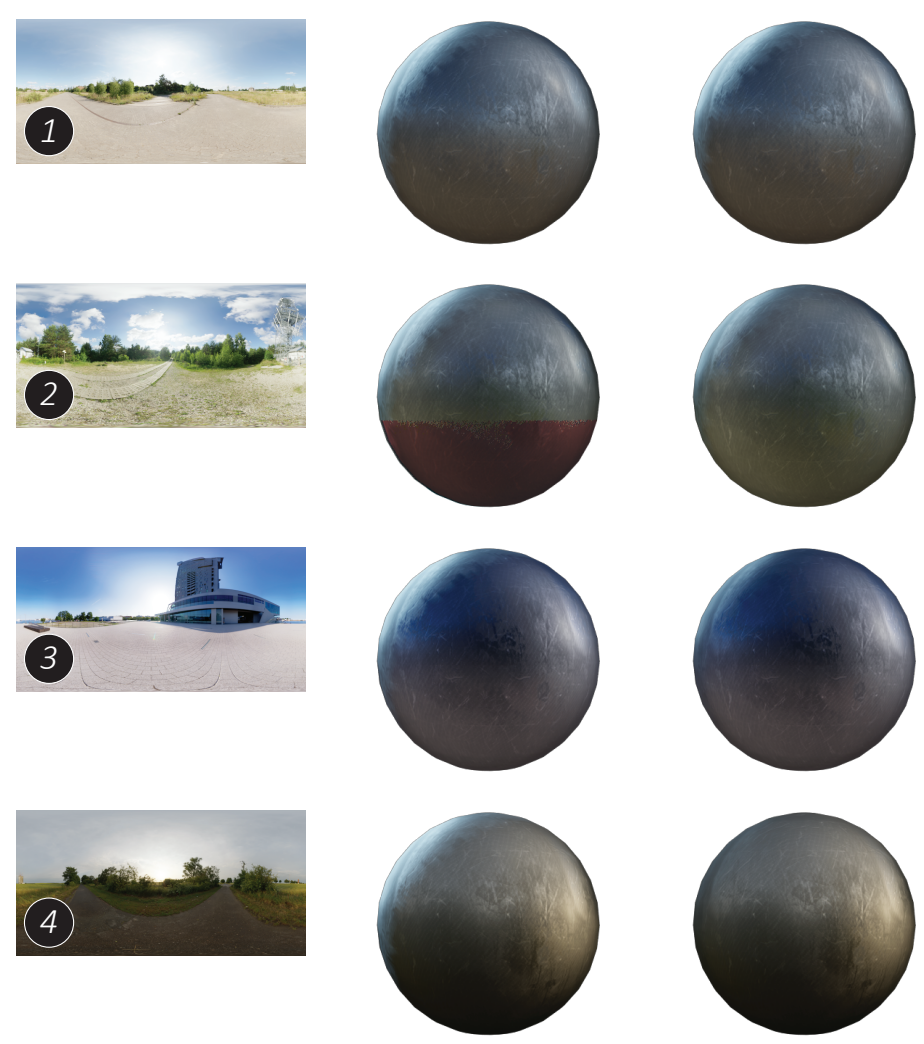

Figure 3.13: Top: EM latent space with rim light intensity values painted such that rim light decreases when transitioning from sunny to sunset EMs (1-4). Below: comparison between a fixed edited appearance, and with our dynamic art-directed appearance. 

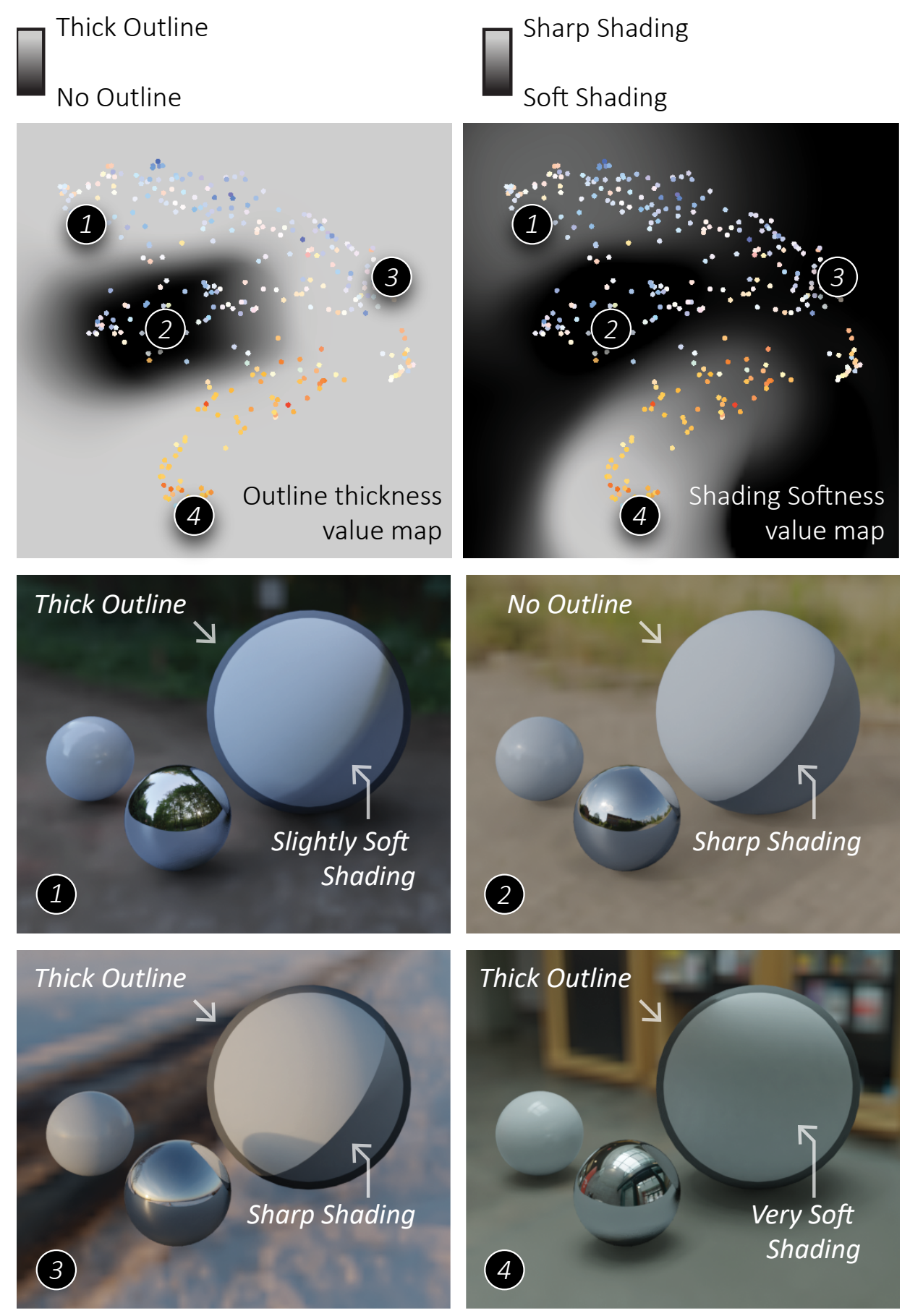

Figure 3.14: Highly varied stylised appearance can be art-directed across different lighting environments. Outline thickness and shading softness in cartoon shading is varied independently. 


\subsection{Limitations and Future Work}

In this chapter we addressed the problem of adapting appearance edits to changing environment lighting. We do this by learning a 2D latent space of EMs. We have shown that, even in $2 \mathrm{D}$, our latent space expresses clear semantic clustering without using explicit categorical labels. Our 2D space allows artists to use painting operations to art-direct appearance attributes across a range of environment lighting. Having shown consistent embedding of new EMs (e.g. using estimated EMs for AR [82, 92]), these artist directives can then be automatically preserved for all similar EMs encountered in live game and AR applications.

Our solution, however, relies on training data that is representative of real-world environments. For example, our solution is limited by the natural scenes occurring in our training set. This means we would not be able to support the complexity of synthetic scenes or custom-built studio lighting environments. Similarly, due to the lack of night-time EMs in our training set, these EMs are not separated from the other outdoor samples in our space.

A few failure cases in our embedding are shown in Figure 3.15. We find some artificial-lighting indoor EMs embedded into the outdoor-overcast cluster. We attribute this to the lights being cooler in colour temperature than those in the indoor cluster. The snowy scene being embedded in the sunny cluster may be due to the lack of these scenes in the training set.

While we show separation of outdoor and indoor EMs, many indoor EMs have multiple lights casting both hard and soft shadows. These exhibit no clear organisation or clustering in our space. We also trained our latent space without assumed semantic labels to avoid ambiguous meanings. In future work we would like to cluster EMs into more categories than just indoor, sunny and overcast. One could leverage semi-supervised learning with labels to do this. We expect this could learn clustering with richer semantic categorisation expressed in the embedding.

This work allows for art-directed rendering under real-world illumination by controlling global material and lighting parameters. However, art direction still 


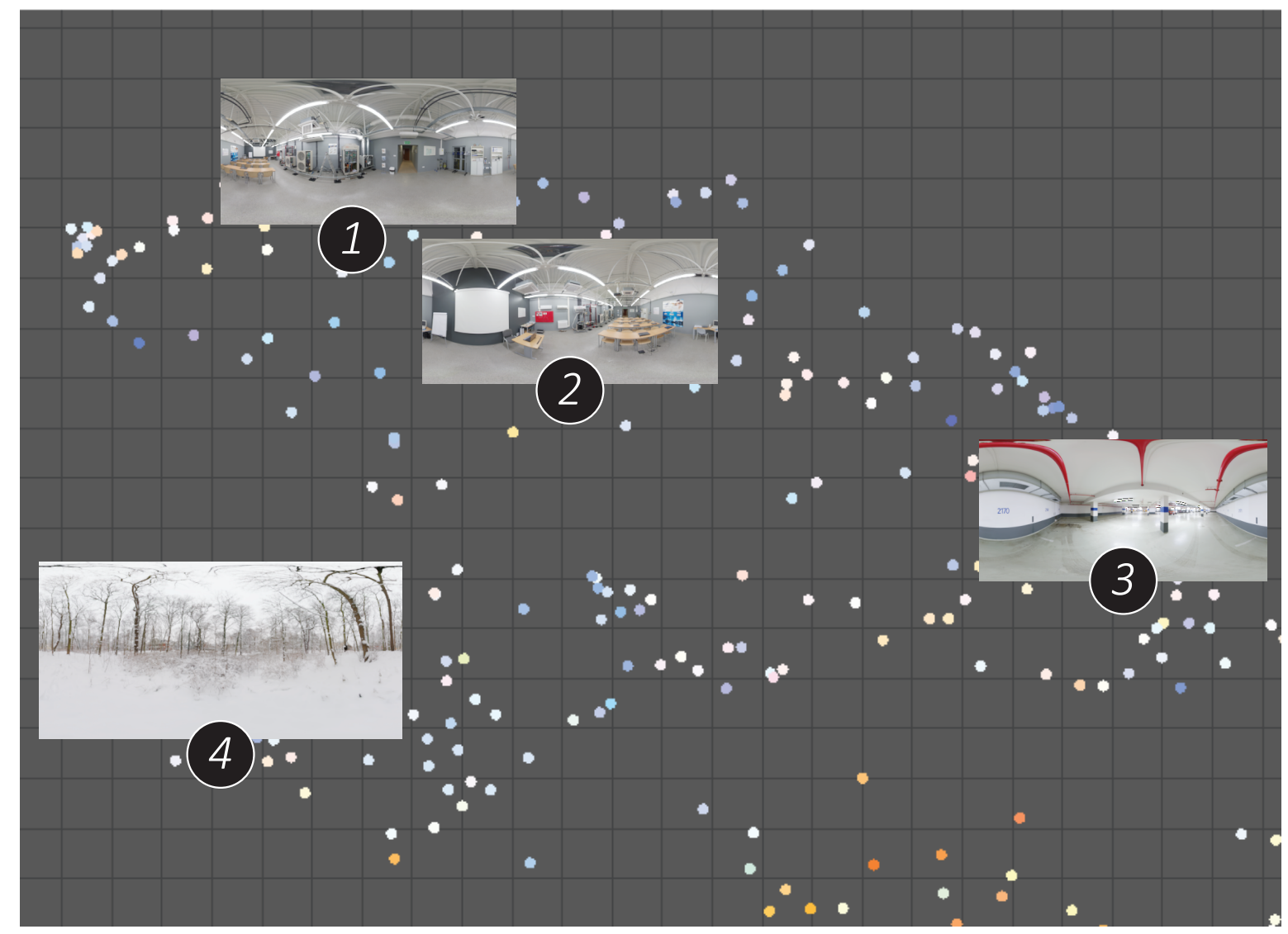

Figure 3.15: Failure cases: Some artificial-light indoor EMs are embedded in the outdoor-overcast cluster (1-3). One snowy overcast EM was wrongly embedded in the sunny cluster. 
requires local control of shadow and highlight shapes. A director will often ask to place lighting and shadows that are fictitious yet artistically convey the intended look. Although this is more important for stylised rendering, shadows are also edited non-physically, in realistic rendering [31]. This chapter addressed automating art direction under varied real-world lighting conditions, but ignores shadow variation as the light direction varies. To handle this case, the next chapter proposes new stylised shadow editing techniques that can be animated to preserve art-directed shading as light direction varies. 


\section{Chapter 4}

\section{Shading Rig: Dynamic Art-Directable Stylised Shading for 3D Characters}

\subsection{Introduction}

Cel animation remains a prevalent form of artistic expression. The freedom offered by manual drawing largely attracts content creators to develop hand-drawn comic and cartoon style characters for feature films and interactive media such as games. Using digital tools, artists create 3D models from character artwork for automated rendering from any viewpoint. However, many manual appearance edits are required to keep 3D stylised rendering faithful to hand-drawn media. This is important for preserving the original appeal and emotional response elicited by the character and story $[100,101,102,103]$.

One of the key challenges of 3D cartoon media is controlling the light and shadow areas generated by conventional toon shading [104]. This is because the abstract two-tone shading style creates undesirable shadow shapes, or removes important surface details, when illumination changes. Even in realistic rendering styles, artists require fine-grained, non-physical, shadow shape control [31]. Stylised feature films thus require frame-by-frame editing during post-production which is inefficient, especially when repeating the same manual edits in different shots. For interactive stylised media and games, post-production is not possible, as frames 
need to be synthesised and displayed at real-time rates, under varying illumination. As a consequence, stylised interactive content features limited interactions, fixed lighting and inflexible camera movements, to keep pre-production costs manageable [102].

Considering the use of 3D cartoon characters will increase drastically, we face greater challenges for stylised interactive media and game creation. A new approach is desired to enable artist-defined toon shading edits that automatically adapt to dynamic lighting in real-time. Unfortunately only a few approaches exist towards this challenge. For example, mesh-based editing methods support fixed lighting, but require further manual tweaking under dynamic lighting [102]. Todo et al. 2007 [3] support keyframing of edits across an animation sequence. However their shadow animation is discontinuous, unless many keyframes are added to fill in the gaps. The flexibility of rotoscoping vector masks, commonly used for shade editing in offline films, cannot support real-time dynamic animation on $3 \mathrm{D}$ surfaces.

Real-time 2D shape interpolation offered by optimal mass transport [4] could solve the discontinuous shadow animation problem. However, this is limited by texture resolution where artists strive for infinite resolution vector quality [102, 103].

We present the "Shading Rig", a framework for designing and animating dynamic art-directed 3D toon shading. We design a novel parametric model for generating shading edits, that allow for continuous, real-time, art-directed shadow animation. In particular, our model can generate edits to existing shade, rather than designing arbitrary shadows from scratch. Unlike the static offset functions of Todo et al. 2007 [3], our edits are directly manipulable, and animatable, while preserving vector-quality detail. Notably, our method allows artists to create and animate edits across a surface mesh, allowing for both time or light changes. Animators can then precisely pre-animate how light and shadow fall on a character as determined by art direction. Interactive applications can then reproduce the artist-defined shading variation, at a much lower performance cost than real-time image-based shape interpolation [4]. Most importantly, as illustrated in Figure 3.1 our shading rig enables dynamically art-directed rendering in interactive cartoon media, without artist intervention. We summarise our contributions as follows: 
- We present a novel parametric model for 3D toon shading edits for real-time, animatable, vector-quality shade editing.

- Our shading edits provide continuous motion and shading shape interpolation, with fewer keyframes than previous approaches such as Todo et al. 2007 [3].

- Our shape model is computationally cheaper than state-of-the-art real-time 2D shape interpolation techniques such as Nader and Guennebaud [4].

The remainder of this paper includes a state of the art review, followed by our approach. Section 4.4 provides a mathematical description of our model, followed by the experimental evaluation and comparison to previous techniques in Section 4.5. Section 4.6 discusses limitations of our technique and proposes future work.

\subsection{Related Work}

In this section we survey prior work in toon shade editing for both offline and realtime applications. We also review patch-based stylisation work for artist-defined shading animation, as well as implicit and texture-based 2D shape interpolation for representing $2 \mathrm{D}$ toon shadow shapes.

\subsubsection{Toon shade editing}

To reduce the likelihood of problematic shadows from conventional toon shading [104], vertex normal smoothing [108] helps to simplify shadows, but this removes high-frequency details. Other mesh-based editing techniques such as normal or UV editing $[102,103]$ require high mesh density and the edit cannot be animated across the surface mesh. Previous works that allow for stylised toon shading are still limited in controllability. 2DToonShade [109] can be used to control toon shading on 2D drawings. The work of Todo et al. 2013 [2] allows a user-created lit-sphere image to arbitrarily define stylised shading gradation on 3D surfaces. However, for complex characters, shading edits on a lit-sphere (or similar parametrisation [110]) 


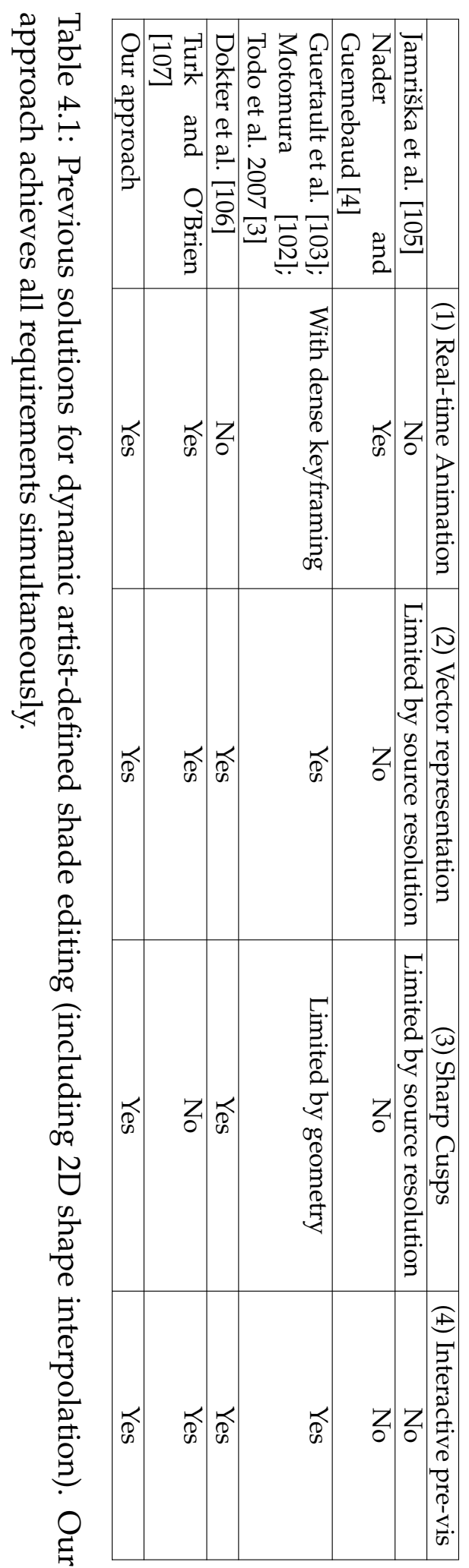


cannot be made locally without unpredictably affecting other areas. Vanderhaeghe et al. [111] use procedural shading primitives to build custom stylised shaders, but these also lack local control. Our problem concerns dynamic toon shading control, rather than producing line details via contour rendering [112].

The paint interface of Todo et al. 2007 [3] allows artists to edit toon light and shade regions arbitrarily on a mesh. Smooth offsets are made to the underlying intensity distribution of the key light. However, its interpolation between keyframed intensity distributions is limited, which can require many keyframes for continuous animation. Arief et al. [113] provide local lit-sphere shading edits while preserving the artist defined shading style. The edits are parametric, allowing for smooth animation, but the shape of each edit is not controllable. Anjyo and Hiramitsu [114] develop parametrically shapable view-dependent specular highlights, suitable for animation. However, we also require stable behaviour for diffuse light and shadow. We also use a parametric model for continuous movement and shape interpolation, but use parameters based on general shading principles, for fine-grained edits beyond specular highlights.

\subsubsection{Artist-Defined Lighting and Stylised Shading}

Many existing techniques allow artistic design or editing of realistic lighting 3D scenes. The method of Okabe et al. [115] allows users to draw the desired realistic illumination on a 3D model. However, it only solves for global lighting instead of local edits. Tada et al. [116] solve local illumination adjustments using RBF interpolation spatially between control points. WYSIWYG NPR [117] lets artists directly draw shading on 3D surfaces as simple line strokes. Several works support freeform editing of occlusion shadowing from 3D meshes [118, 119, 120]. Many other art-directable lighting methods also allow artists to non-physically control physical rendering phenomena [27]. These produce plausibly realistic images whereas toon shade editing often requires implausible shadow shape manipulation.

Artist-defined stylised shading and animation has been achieved with patch-based methods. One such method is image analogies [121] for stylising animation [122] 
and video [105] using image features. Given a few manually stylised video frames, these methods can preserve local stylisation across an image sequence. However, they are not real-time, and are limited to fixed lighting. Real-time adaptions of this approach also only support global shading $[26,123]$ with no accomodation for local editing.

\subsubsection{D Shape Interpolation}

We also survey prior work in 2D shape interpolation as a potential solution to stylised shadow shape interpolation. Level sets of an implicit radial basis function $(\mathrm{RBF})$ representation have been used to smoothly interpolate between arbitrary 2D shapes [107]. However, these fail to reconstruct sharp cusps. While analytic 2D signed distance fields (SDFs) can reproduce sharp points, and smoothly blend together, these only produce limited geometric shapes. Recently, Seyb et al. [124] proposed to freely deform 3D SDFs using a convex hull, but this has not been adapted to $2 \mathrm{D}$ shapes.

Animatable 2D vector masks are commonplace for freeform 2D shape design and direct manipulation, with infinite resolution. These are used in an offline 2D compositing or rotoscopy context to create and edit shade in 3D cartoon animation [125]. Vector Shading Curves [126] have been used to define stylised shading details but only in 2D. Eisemann [127] convert toon shaded 3D renderings into vector art. Loop and Blinn [128] encode 2D vector paths on triangular meshes providing vector shape rendering under arbitrary 3D projective transformations, suited for GPU rendering [129]. While real-time on the GPU, this method offers limited dynamic shape manipulation without costly re-computation of the mesh. To our knowledge, current research cannot support animated vector texture mapping in real-time applications (only precomputed static vector textures $[130,131,132,106])$.

Fully controlled shading can be rendered as a hand-drawn static texture map but cannot respond to lighting changes. Optimal mass transport can provide this texture animation using displacement interpolation [133, 134]. For realistic images, this has been used for realistic lighting transfer on faces [135]. Recently, Nader and 
Guennebaud [4] have achieved real-time performance for approximate mass transport. Although this provides fast arbitrary shape interpolation, there is still dependence on image resolution. While higher resolutions can reduce pixelation artifacts upon magnification, the sampling rate of image textures mapped on 3D meshes would be inconsistent at different viewpoints. Production game artists also go to great lengths to maintain vector quality to support extreme close-ups [102], so we target a fully vector-based approach.

\subsubsection{Summary of Prior Work and Comparison}

We summarise the limitations of prior work, as related to production requirements. Based on industry use cases [102, 103], we highlight four main requirements for interactive stylised media:

1) Real-time: Toon shading must render and animate in real-time and minimally impact computational resource budget.

2) Vector representation: Shading boundaries must be sharp, without pixelation artifacts, regardless of viewing distance.

3) High-frequency detail: Sharp cusps must remain sharp upon close-up, independent of mesh/texture resolution.

4) Interactive pre-visualisation (pre-vis): editing, animation and pre-visualisation of shade variation must minimally impact artist turn-around time.

In table 4.1 we compare the state-of-the-art from each approach against these requirements. In this table we note that mesh editing $[102,103]$ and the method of Todo et al. 2007 [3] can only reproduce sharp cusps as long as mesh density and topology allows. Without a high density mesh, this limits the shadows to simple polygonal shapes which restricts artistic expression. Similarly, we classify the patch-based method of Jamriška et al. [105] to have texture independence and sharp cusps, due to its ability to sample high-frequency patches in the source textures without blurring due to magnification. We consider the real-time shape interpolation method of Nader and Guennebaud [4] to fail the interactive pre-visualisation requirement. Using higher resolution images dramatically 

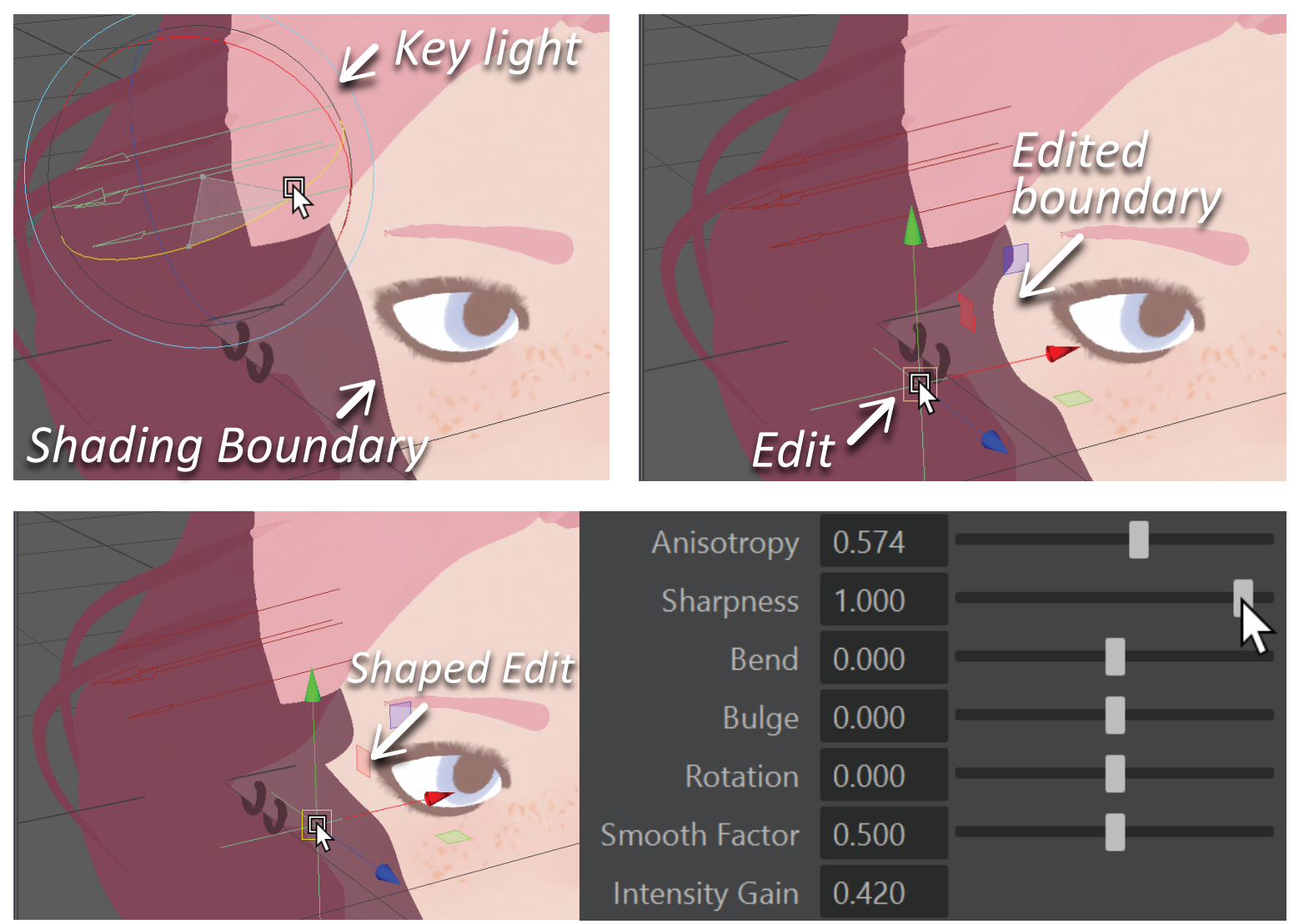

Figure 4.1: Top-left: key light adjusted for base toon shadows to be edited. Top-right: edit placed near surface mesh to adjust shading boundary. Bottom: parameters controlling the shape of the edit (in this case, increasing Anisotropy and Sharpness creates the desired sharp contour).

increases this method's necessary pre-computation time. Pre-visualising an animation would then become non-interactive, and in turn prohibitive for practical use. Our approach is the first to simultaneously support all the above requirements. We achieve this by designing a parametric model based on artistic shading principles identified in the next section.

\subsection{Shading Rig Overview}

In this section we give an overview of our shading rig workflow and interface, to shape and animate toon shading. We also design our parameters for generating 


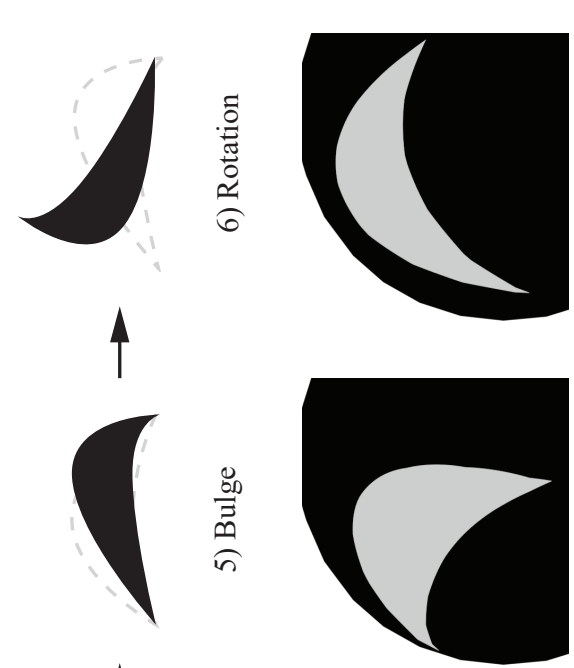

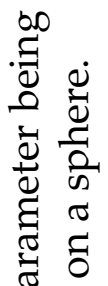

‥ 동

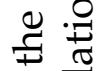
善.气 了 艺 छ \& ๒ొ ฮั)

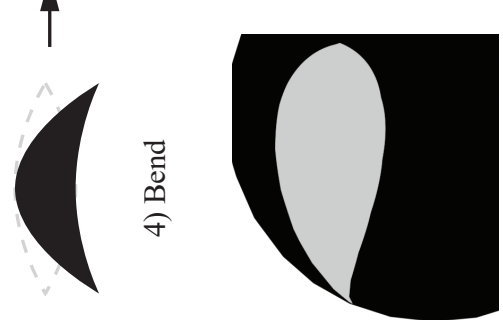
क . 艺 $\div$ ช के छ 둥 올

$\uparrow$
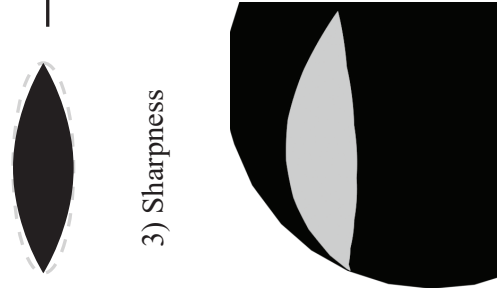

릉

ప.

द

농

दृ

का

s 3

छ

$\uparrow$
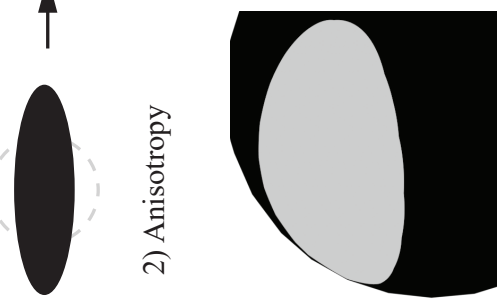

它

क क

을

롱

तี ज

또.응

$\dddot{3}$ 定

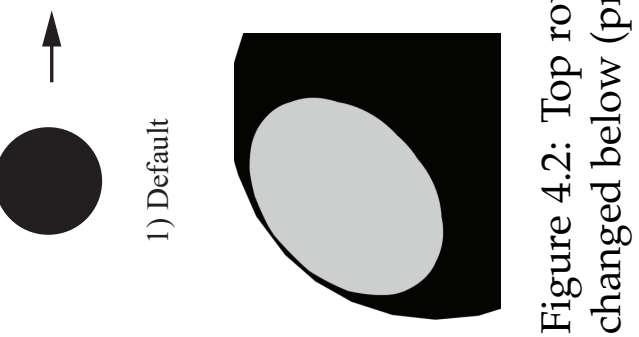



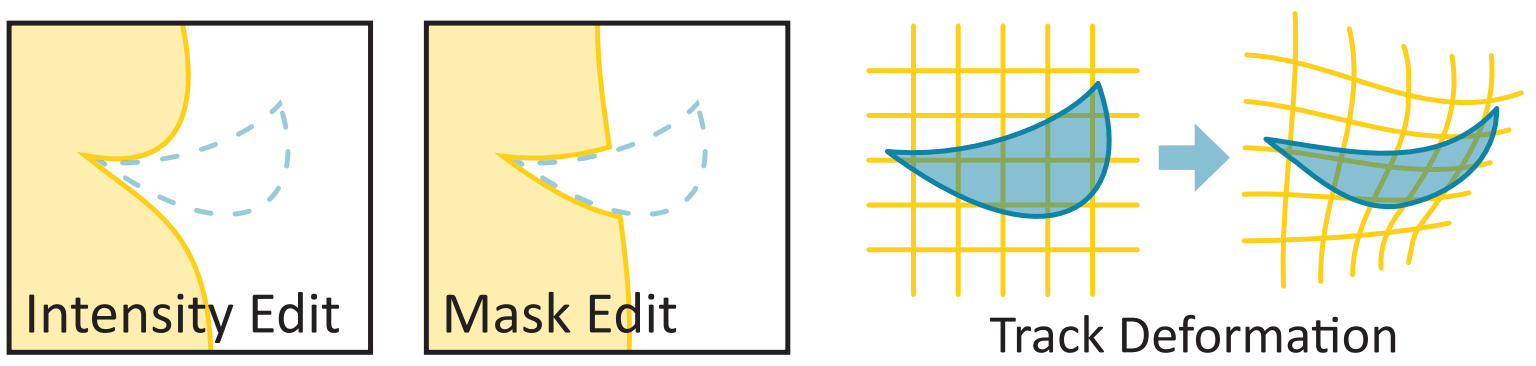

Figure 4.3: Modes for blending edits together, and tracking surface deformations during animation.
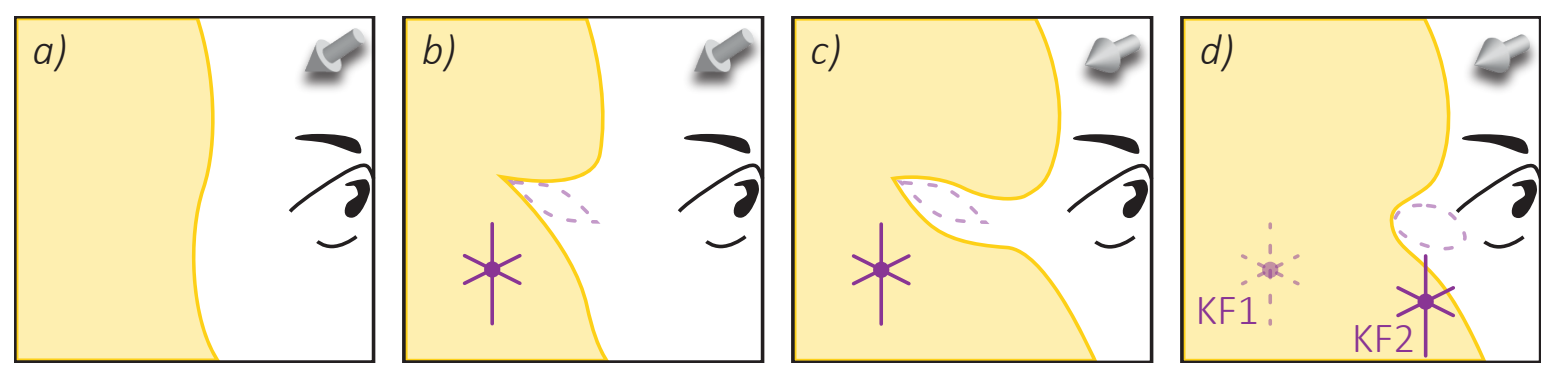

Figure 4.4: Steps to animate a single edit with lighting changes. a) Unedited shading from key light. b) Shading rig edit placed to edit shading boundary. c) Key light rotated and shading must be edited again. d) First edit is keyframed to move and change shape under new lighting. The edit's position and shape interpolate between keyframes KF1 and KF2 as the key light direction changes.

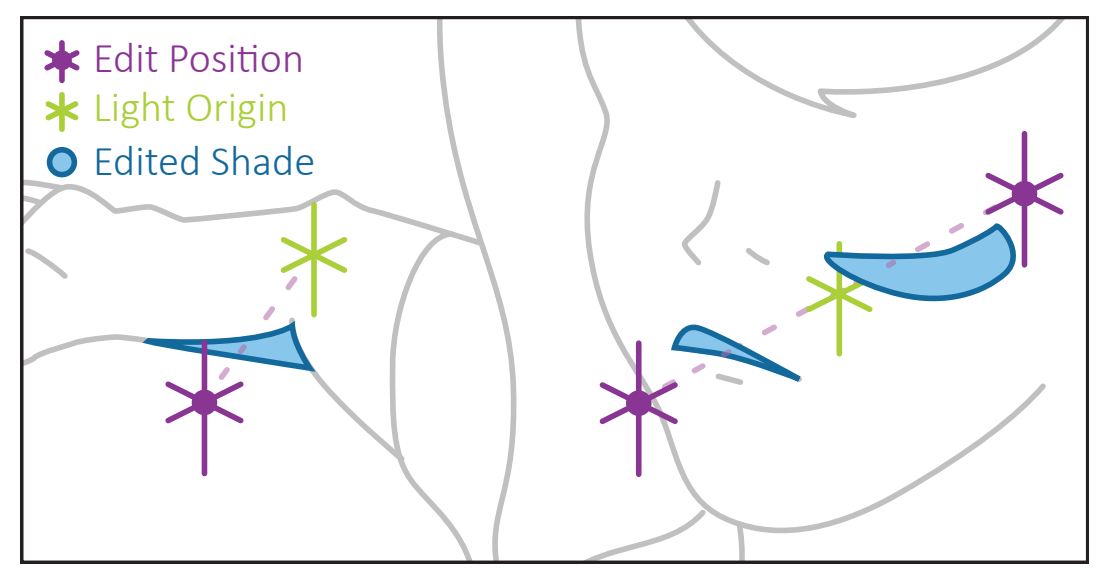

Figure 4.5: Using multiple light origin points when editing shade across different areas on a character. 
toon shade edits, considering basic light and shadow elements described in artistic shading literature (Section 4.3.3). Section 4.4 gives the formulation of the features described in this section.

\subsubsection{Shade Editing Workflow}

Inspired by the key light and fill light paradigm familiar to artists [18, 136], we define discrete shading edits, controlled like point lights but with shapeable intensity distributions. A directional light is firstly created as the key light providing the base shading on a 3D mesh. Then our edits are used like shapeable fill lights for local shading adjustments. Artists control the shape of an edit using the parameters which we define in Section 4.3.3. The edits are scaled in size based on their distance to the surface, to match the behaviour of point lights in toon shading.

With multiple edits, shapes can be built with the expressivity of offset painting Todo et al. 2007 [3], but can be tweaked and animated with the flexibility of conventional lighting. We refer to the collection of edits, and their parameters, as the "shading rig". Analogous to artist-designed rigs with 3D controllers (commonplace for animating characters [137]), our shading rig allows artists to animate stylised shading with familiar tools. Figure 4.1 shows this shade editing workflow, using our prototype interface in Maya. In this example, an edit (represented by a locator in 3D space) is brought to the surface of the mesh. This first expands the lit region around the edit radially, the default behaviour of point lighting in toon shading. The edit's shape parameters can then finely control the boundary of light and shade.

\subsubsection{Animation Workflow}

To preserve art direction under dynamic lighting, the shading rig is animated with changes in the key light direction. Artists first edit toon shading for a given key light direction, using our edits. When the key light is rotated, the shadow boundary 
changes. The shading rig is then adjusted to achieve the desired shading in this new light direction. The adjustment to the shading rig is keyframed at this light direction. Section 4.4.2 describes how we interpolate the shading rig between light directions.

The shading rig interpolates the edited shadow when the key light is rotated between the keyframed directions. Artists add more keyframes for different key light directions as needed to meet art direction. In real-time visualisation, the shading rig keyframes are interpolated between keyframed light angles to achieve dynamic shade adjustment under changing lighting. We visualise this process for a single edit in Figure 4.4. Figure 4.4c) illustrates the problem that static edits become invalid when lighting changes, and must be dynamically corrected like in Figure $4.4 \mathrm{~d})$.

\subsubsection{Shading Edit Parameters}

In the literature on artistic shading principles used by professional artists, Hogarth [138] distils individual two-tone shapes of light and shadow in the following ways. Abstract, rounded shadow shapes are described as "islands of minimal light", "droplets of light" for selectively expressing certain forms. Rounded "deep pools of shadow" are described to shadow eyes under the brow of a head. More directional shapes are described as "linear highlight[s]" or resemble "arcs of light" or a "curving slash" that "thickens at certain points", and "tapers and gradually disappears". Based on these descriptions we design parameters below. The behaviour of these parameters on an edit's shape is shown in Figure 4.2.

First, for rounded "pools of shadow", circular shapes are produced by our model by default. In our framework this is equivalent to using point light sources to add radial light locally on a surface. The Intensity Gain parameter controls the shape's influence on shading. This is similar to controlling intensity in conventional lighting. We allow negative intensities to create shadows.

We add an Anisotropy parameter, to elongate circular shapes for the "linear highlight $[\mathrm{s}]$ ", and a Sharpness parameter for edits to "taper" at the ends. Varying 
this parameter smoothly transitions between rounded and sharp endpoints. We add a Bend parameter to curve the elongated shapes into the "curving slash[es]" and "arcs of light". Its magnitude controls the degree of shape curvature. For curved shapes that "[thicken] at certain points", we provide a Bulge parameter. While Anisotropy alters thickness, Bulge asymmetrically controls where thickening occurs along a curve.

A Rotation parameter allows rotating the shape to align it with surface details. Each edit is also given a Softness parameter to achieve soft boundary edges in local regions, as required when depicting certain rounded forms [138, 103].

To control the influence of surface details on the shape, we introduce a Normal Smooth parameter to smooth surface normals for simplified shading [108]. Increasing the Normal Smooth value will smooth out surface details where simpler shading is desired. Decreasing its value helps the shape to conform with surface details. This behaviour allows an edit to "repeat the external contours" [138] to reveal ridges and complex detail on a surface. The normal smooth parameter also helps to achieve complex or simple shading, as varied depending on character traits [139].

\subsubsection{Edit Blending and Deformation}

Custom shapes beyond the range expressed by our parameters are achieved by combining or blending individual edits. Our framework uses two types of edits for blending: intensities and masks. Intensity edits produce shapes that smoothly blend with base shading, and with other edits. These edits create smooth joins between other shaded areas. Masks edits, however, create binary regions of light and shade that use boolean union and subtract operations to blend with other shading regions. This helps fine-scale edits hold their shape under lighting variation (unless they are specifically animated with the key light). These edits create sharp joins when combined with other shaded areas.

Another useful mode for each edit is Track Deformation, which fixes an edit's shape to a moving or deforming surface during animation. Both Intensity and Mask type 
edits can be set to track surface deformations. This saves artists from having to manually animate edits to follow a deforming surface. It also restores the static behaviour of texture or vertex painting while remaining animatable like a point source. Our edit blending modes and deformation tracking behaviour are visualised in Figure 4.3.

\subsubsection{Local Editing Control}

Like conventional point lighting, each edit has a user-defined radius of influence, beyond which the edit's intensity falls off and has no influence on shading. With this distance-based fall-off and the previously mentioned shape scaling control, animating edits to smoothly appear and disappear is achieved by moving them toward or away from a surface. For Intensity edits, the Intensity Gain parameter can also be used.

Since our edits can be shaped, we require a direction in which to project the shaped areas of light and shade. By default we direct the projection toward the centroid of the mesh. For complex editing on geometry features away from the centroid, users specify a new point we call the light origin. Moving the light origin allows edits to project shaped lighting toward the region of interest. The light origin also controls where normal smoothing occurs, as explained in the next section.

Many light origin points can be used. For example, edits made on a character's head would need a separate light origin, than for edits on the arm. As shown in Figure 4.5 , several light origin points, assigned to different edits, can be used in different regions of the character.

We integrate the functionality into an existing 3D application workspace such as Maya or Blender, which facilitates 3D manipulation, real-time preview, and animation. Within the 3D application, users are able to animate the position and shape parameters of each edit, to achieve the desired shadow movement under dynamic lighting. In the next section we give the mathematical formulation of our shading rig model to achieve the functionality described here. 
Table 4.2: Shading shape model control parameters.

\begin{tabular}{|c|c|c|}
\hline Parameter & Symbol & Effect on Shape \\
\hline Anisotropy & $a$ & Elongates the shape horizontally. \\
\hline Sharpness & $s$ & $\begin{array}{l}\text { Creates round or sharp endpoints for the } \\
\text { elongated shape. }\end{array}$ \\
\hline Bend & $w_{y}$ & Curves the elongated shape. \\
\hline Bulge & $w_{x}$ & $\begin{array}{l}\text { Asymmetrically bulges one side of the } \\
\text { elongated shape. }\end{array}$ \\
\hline Rotation & $\theta_{r}$ & $\begin{array}{l}\text { Rotates the final shape for different } \\
\text { orientations. }\end{array}$ \\
\hline Normal smooth & $\tau$ & $\begin{array}{l}\text { Controls how much geometry detail is } \\
\text { preserved, or smoothed out, in the shape. }\end{array}$ \\
\hline Intensity Gain & $G$ & $\begin{array}{l}\text { Intensity edit's influence on the existing } \\
\text { shading. }\end{array}$ \\
\hline Softness & $d$ & $\begin{array}{l}\text { Mask edit's boundary softness for smooth } \\
\text { gradation between light and shade. }\end{array}$ \\
\hline
\end{tabular}

\subsection{Shading Rig Edit Model}

This section firstly describes the mathematical formulation of our shading rig model, given the parameters and interface described in the previous section. Later, we describe how we implement deformation tracking, and animating the shading rig with lighting changes. For reference we list all the user parameters in Table 4.2. A full table of all symbols and notation is given in the Appendix.

We map the shape of each edit to a local texture space projected on a surface mesh. The shape is generated by a warped intensity distribution and then thresholded for a binary mask of light and shade.

While 3D software provide texture projection interfaces for local decal placement, we implement a simple texture space behaving similar to point lighting, in the Appendix. Specifically, this allows our parametric distribution (Section 4.4.1) to exhibit the distance-based shape scaling mentioned in the previous section.

The texture space is translated across the surface by a moving a point $\mathbf{p}_{l}$ in $3 \mathrm{D}$ space (following the analogy of moving a point light at position $\mathbf{p}_{l}$ ). We continually direct the texture space projection toward the light origin point $\mathbf{p}_{o}$. As mentioned in the 
previous section, this is set to the mesh centroid by default, but is moved by the artist as necessary.

This local texture space for an edit is denoted by $(u, v)$, and is a function of $\mathbf{p}_{l}$ and the world-space position of the surface shading point $\mathbf{p}_{w}$ (formulation given in the appendix).

\subsubsection{Intensity Distribution}

Given our parameters in Section 4.3.3, we aim to generate these previously described shapes for toon shadow editing. To achieve shape manipulation with our parameters, and smooth blending with existing shade, our shading rig edits take on a shape described by a modified bivariate gaussian intensity distribution mapped to the local texture space coordinates $(u, v)$.

\section{Anisotropy ( $a$ ) and Sharpness ( $s$ )}

For one edit, we define an intensity distribution $I$, given a point $(x, y) \in \mathbb{R}^{2}$ as follows:

$$
I(x, y)=e^{-\alpha x^{2}-\frac{1}{\alpha}|y|^{2-s}}
$$

where $s \in[0,1]$ is the Sharpness parameter, and $\alpha=1-a$ given the Anisotropy parameter $a \in[0,1)$. This gives a smooth intensity distribution with the ability to elongate (using Anisotropy), and sharpen the elongated points (using Sharpness). This formulation allows the sharpness parameter to generate mathematically sharp cusps, independent of texture resolution or mesh density. This achieves the first three steps in the shape manipulation process shown in Figure 4.2.

\section{Bend $\left(w_{y}\right)$, Bulge $\left(w_{x}\right)$, and Rotation $\left(\theta_{r}\right)$}

We implement the behaviour shown in the last three steps in Figure 4.2, using the following formulation. For the desired curvature and asymmetry behaviour, we sample $I(x, y)$ with warped $(u, v)$ coordinates $\left(u_{w}, v_{w}\right)$. The Bend and Bulge 
parameters are denoted by $w_{y}$ and $w_{x}$ respectively. Defining $\mathbf{w}:=\left(w_{x}, w_{y}\right)^{\top}$ and $\mathbf{u}:=(u, v)^{\top}$, we first compute a warping function $\theta_{w}(\mathbf{u})$ as:

$$
\theta_{w}(\mathbf{u})=k_{w} \mathbf{w} \cdot\left(\boldsymbol{R}\left(\theta_{r}\right) \mathbf{u}\right)
$$

where $k_{w}$, the degree to which $w_{x}$ and $w_{y}$ distorts $I(x, y)$, is set to $10 . \boldsymbol{R}(\theta)$ is the $2 \mathrm{D}$ rotation matrix given an angle $\theta$. Given this value of $k_{w}$, typical values of $w_{x}$ range between \pm 0.5 while $w_{y}$ would be set between \pm 0.1 .

Given $\theta_{w}$, and the shape rotation $\theta_{r},\left(u_{w}, v_{w}\right)$ is computed as:

$$
\left(u_{w}, v_{w}\right)=\mathbf{w}+\boldsymbol{R}\left(\theta_{w}(\mathbf{u})\right)\left(\boldsymbol{R}\left(\theta_{r}\right) \mathbf{u}-\mathbf{w}\right)
$$

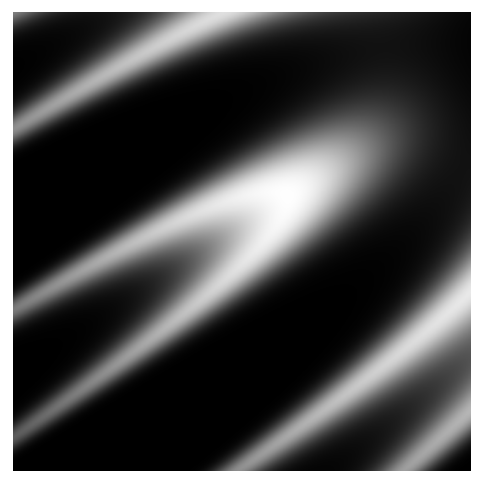

(a)

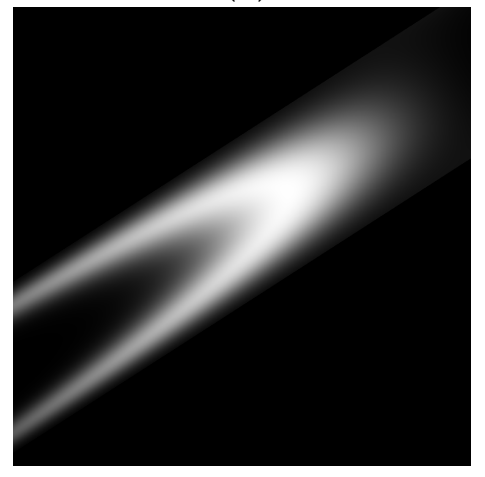

(c)

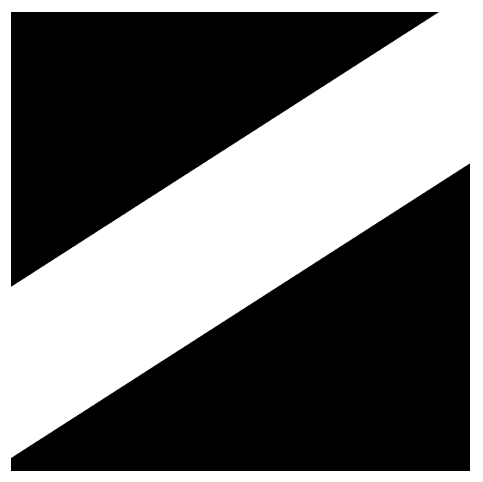

(b)

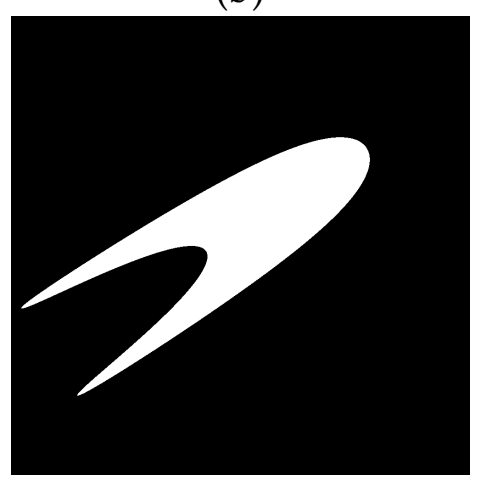

(d)

Figure 4.6: (a) Original intensity distribution $I\left(u_{w}, v_{w}\right)$ with periodicity artifacts when using a large Bend value. (b) Mask created by $c_{w}\left(\theta_{w}\right)$. (c) Intensity distribution masked by (b) which removes the artifacts. (d) Final shape after thresholding. 
With this modification to $(u, v)$, Bend will curve $I(x, y)$ upwards $\left(w_{y}<0\right)$ or downwards $\left(w_{y}>0\right)$ along the y-axis. Bulge will warp $I(x, y)$ left $\left(w_{x}>0\right)$ or right $\left(w_{x}<0\right)$ along the $\mathbf{x}$-axis, asymmetrically bulging either side after increasing Anisotropy. The Rotation parameter $\theta_{r}$ rotates the $\mathrm{x}$ and $\mathrm{y}$ axes before the Bend and Bulge operations take effect.

Our formulation in Equations 4.2 and 4.3 roughly describes rotating the coordinates $(u, v)$ about the point $\left(w_{x}, w_{y}\right)$ at a spatially varying angle $\theta_{w}(u, v)$. The magnitude of $\theta_{w}$, and thus the distortion, increases along the $u$ and $v$ axes at the rate of the Bulge $\left(w_{x}\right)$ and Bend $\left(w_{y}\right)$ values respectively, providing the desired curving behaviour.

Each edit's intensity distribution, is then pre-multiplied by several factors to remove repetition artifacts from warping, limit the range of influence, and control the influence of surface details on the edit's shape. Firstly, to mask out repetition artifacts in $\left(u_{w}, v_{w}\right)$ from periodicity, we use a mask $c(\theta)$ where $c(\theta)=1$ if $-\frac{\pi}{2}<\theta<\frac{\pi}{2}$ and $c(\theta)=0$ otherwise. This limits the influence of $\theta_{w}$ outside the range $\left[-\frac{\pi}{2}, \frac{\pi}{2}\right]$. The repetition artifacts and mask are shown in Figure 4.6.

Then, the range of influence of each edit is limited to the user-specified distance $R$ away from $\mathrm{p}_{\mathrm{l}}$, similar to point lighting. We attenuate the edit's intensity distribution using a distance fall-off curve $f_{s}(x)$, with a step-edge width of $k_{s}=0.05$. This intensity attenuation factor $\omega$ is given by $\omega=f_{s}\left(\frac{1}{k_{s}}\left(R-\left\|\mathbf{p}_{\mathbf{w}}-\mathbf{p}_{\mathbf{l}}\right\|\right)\right)$. The fall-off curve $f_{s}(t)$ is taken to be a smooth-step function given by the cubic polynomial curve $3 t^{2}-2 t^{3}$, when $t$ is between 0 and 1 .

\section{Normal Smooth $(\tau)$}

For edit shapes to be more or less influenced by the surface geometry, we also attenuate each edit's intensity by a weight $\gamma$ controlled by the Normal Smooth parameter $\tau \in[0,1]$. We implement surface normal smoothing as a weighted average $\mathbf{N}(\tau)$ between the surface normal $\mathbf{n}$ and smooth sphere-like normals computed from the mesh's centroid $\mathbf{p}_{\mathbf{c}}$ [108]. We compute this average as $\mathbf{N}(\tau)=\mathbf{n}^{\prime}(\tau) /\left\|\mathbf{n}^{\prime}(\tau)\right\|$, where $\mathbf{n}^{\prime}(\tau)=(1-\tau) \mathbf{n}+\tau \frac{\mathbf{p}_{\mathbf{w}}-\mathbf{p}_{\mathbf{c}}}{\left\|\mathbf{p}_{\mathbf{w}}-\mathbf{p}_{\mathbf{c}}\right\|}$

The weight $\gamma$ is then given by $\gamma=\mathbf{N}(\tau) \cdot \mathbf{v}$, where $\mathbf{v}$ is the normalised direction 
vector from $\mathbf{p}_{w}$ to $\mathbf{p}_{l}$. We show the behaviour of local normal smoothing variation in Figure 4.7. As mentioned, edits made away from the centroid are given a light origin controller. For such edits we substitute $\mathbf{p}_{\mathbf{c}}=\mathbf{p}_{\mathbf{o}}$ so that smoothing occurs about this local region.

\section{Intensity edits}

As mentioned in Section 4.3, our Intensity edits blend with existing toon shading boundaries. Mask edits instead preserve sharp joins when combined with other shading, and are unaffected by light changes unless animated with the key light. To combine Intensity edits with existing toon shading from conventional 3D lighting, we sum this existing reflectance $d_{0}$ and the weighted intensity distributions of all $N_{I}$ Intensity edits in the shading rig. A global toon shading threshold $T_{0}$ is then applied. $T_{0}$ is provided by the existing toon shader [104] which the shading rig is applied to. The lit region on a surface, which we denote as $\mathbf{B}_{1}$, is the set of all points that satisfy the following condition:

$$
T_{0}<d_{0}+\sum_{i=1}^{N_{I}} G_{i} c\left(\theta_{w_{i}}\right) \omega_{i} \gamma_{i} I_{i}\left(u_{w_{i}}, v_{w_{i}}\right)
$$

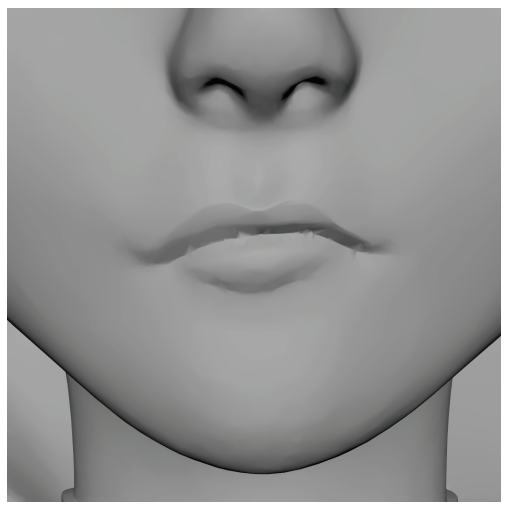

(a)

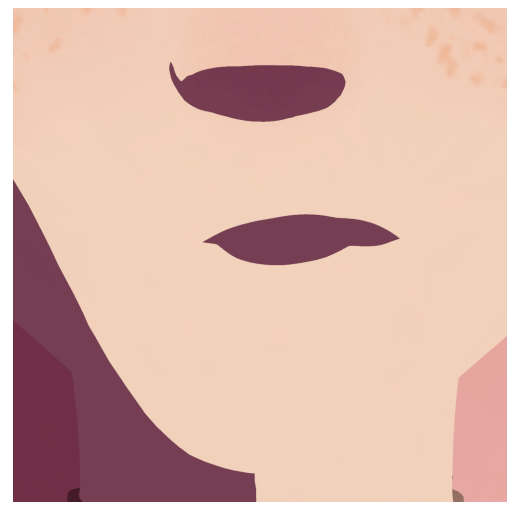

(b)

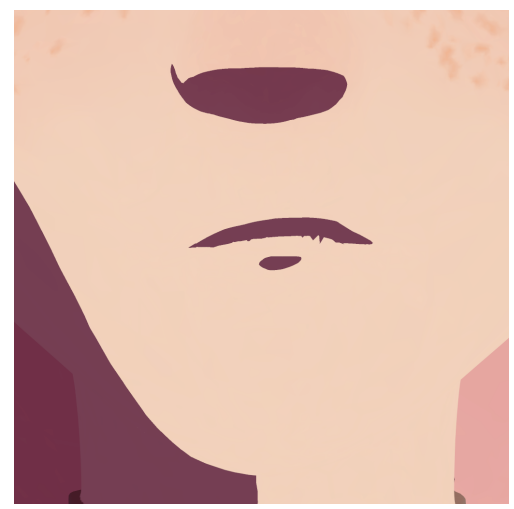

(c)

Figure 4.7: Given the surface mesh in (a), smoothed normals reduce toon shadow artifacts, but remove important shape details. Edits are placed to indicate the nose mouth in (b). Reducing the Normal Smooth parameter for the mouth edit selectively added detail which the model alone did not express. 


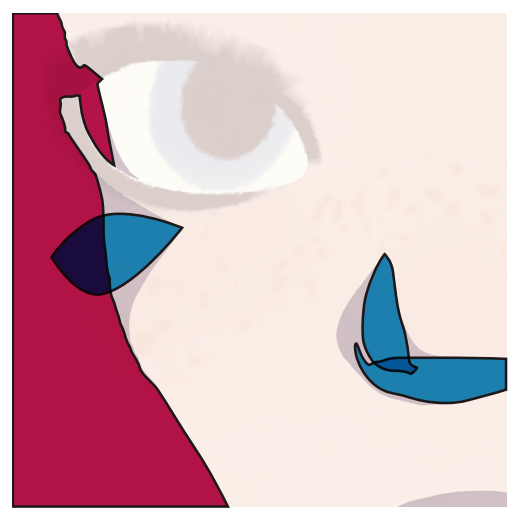

(a)

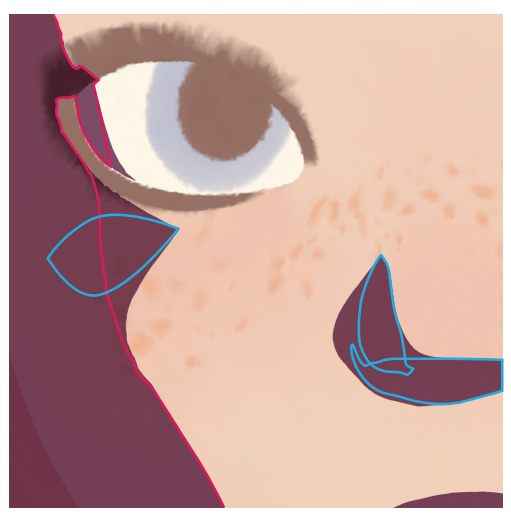

(b)

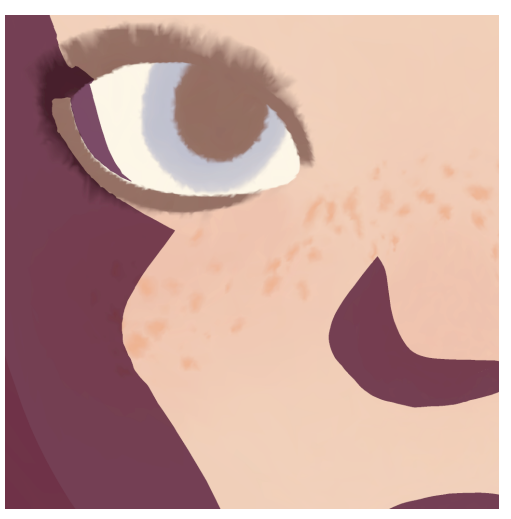

(c)

Figure 4.8: In (a), conventional toon shadow is the red shape lacking cheek and nose definition. Our edit shapes, shown in blue, are placed to depict more detail. In (b) we see the shadow shapes (outlined) are smoothly combined into the desired shading. The final shading is shown in (c).

Here, $I_{i}(x, y)$ is the intensity of the $i^{\text {th }}$ Intensity edit placed in the scene. As written, $I_{i}(x, y)$ is also weighted by a corresponding, user-specified, intensity gain parameter $G_{i} \in \mathbb{R}$. The magnitude of $G_{i}$ controls the strength relative to other shading terms, while its sign affects whether light $(G>0)$ or shadow $(G<0)$ is created. As mentioned, this is analogous to controlling the intensity of conventional 3D lighting while allowing negative values for shadows. In Figure 4.8 we show how our intensity edits can be smoothly combined for toon shadow editing.

\section{Mask edits}

For mask edits, each edit creates their own lit region by thresholding their own intensity fields. Thus the lit region of the $i^{\text {th }}$ Mask edit, which we denote as $\mathbf{M}_{i}$, is the set of points where $t_{m}<c\left(\theta_{w_{i}}\right) \omega_{i} \gamma_{i} I_{i}\left(u_{w_{i}}, v_{w_{i}}\right)$. The threshold $t_{m}$ is set to 0.8 for all mask edits. The lit region of all mask edits combined with all intensity edits is simply: $\mathbf{B}_{1} \cup\left(\bigcup_{i=1}^{N_{M}} \mathbf{M}_{i}\right)$, where $N_{M}$ is the number of Mask edits used in the shading rig.

Any mask edit can be specified to produce a shadow instead of light. For all mask 
edits used for shadow, the modified region is instead given by $\mathbf{B}_{1} \cap\left(\bigcup_{i=1}^{N_{M}} \overline{\mathbf{M}_{i}}\right)$, where $\overline{\mathbf{M}_{i}}$ is the complement of the set $\mathbf{M}_{i}$.

When using multiple light origins, each edit evaluates their own intensity distributions using the $p_{o}$ of their assigned light origins. We then combine the edits using the same equations.

\section{Softness (d)}

The Softness parameter, denoted by $d$ of an edit will smooth its shape's boundary. A smooth boundary of width $d$ is achieved using the same smooth step function $f_{s}(x)$, mentioned previously. For the $i^{\text {th }}$ mask edit, the smoothed edit shape mask is given by $f_{s}\left(\frac{1}{d_{i}}\left(c\left(\theta_{w_{i}}\right) \omega_{i} \gamma_{i} I_{i}\left(u_{w_{i}}, v_{w_{i}}\right)-t_{m}\right)\right)$.

\section{Deformation Tracking}

The deformation tracking function described in Section 4.3 .4 is achieved by evaluating our edits in undeformed object space. For edits that are chosen to track deformation, we substitute the world space position $\mathbf{p}_{w}$ and normal $\mathbf{n}$, with the undeformed object space position and normals, when computing the texture space and intensity distributions.

\subsubsection{Shading Rig Animation}

A shading rig is built from multiple edits based on these shapes. With this rig being fully parametric, artists can freely animate the motion and shape variation using keyframing. One can set keyframes of edit positions and shape parameters on the timeline for a traditional animation sequence. Alternatively, for the case of varying lighting, we develop a system for keyframing with respect to the key light direction. Artists can then animate the shading rig to dynamically adjust conventional toon shading under a changing light direction. We refer to this as pre-animating the shading rig.

At a given light direction, artists adjust the shading rig for their desired result, and 
create a keyframe. Note that these keyframes are set for each light direction, not on a timeline in the traditional sense. Keyframes hold the state of the shading rig, including the 3D position of each edit and their parameter values, at a given light direction. Since our interpolation domain is the key light direction vector, instead of a scalar time value, we use spherical radial basis functions (RBFs) [140]. This preserves the geodesic distance between light direction vectors when interpolating between keyframes. Using the spherical gaussian as the RBF kernel gives smooth interpolation between keyframes. The spherical gaussian function we use is given by:

$$
\exp (\lambda(\boldsymbol{\eta} \cdot \boldsymbol{\xi}-1))
$$

where $\boldsymbol{\eta}$ is the unit vector in the direction being sampled, $\boldsymbol{\xi}$ is the center axis, and $\lambda$ determines the kernel width. We set $\lambda=1$ by default, though this can be changed by the artist to control the interpolation result.

Each edit requires at least two keyframes for interpolation between key light directions. By default, the RBF interpolated function will extrapolate how the shading rig behaves when the key light rotates away from these light directions. Artists can add more keyframes to control the result, or modify the RBF kernel width $\lambda$. As in general scattered data interpolation using RBFs, large $\lambda$ values provide smoother interpolation between distant keyframes, while small $\lambda$ values will prevent overshoot between close keyframes.

When pre-animating the shading rig, the same number of edits are used for all key light directions. Edits that appear and disappear must still exist in the scene. As mentioned, this effect is controlled by the distance-based intensity falloff and shape scaling, or with intensity gain for intensity edits.

\section{Art-directed Shading for Dynamic Objects}

Rather than the light direction changing, an object may rotate relative to a key light direction. In terms of shading, this case is equivalent to the light rotating in the opposite angle relative to the object. To reproduce the correct shading at this 
orientation, we can simply sample the shading rig animation at this opposite angle, given by the current key light direction and the orientation of the object. We then update the shading rig at this light vector but apply the shading in object space, like we do for deformation tracking, ensuring edits that do not change with the key light remain static.

Based on this animation and interpolation, we show an example pre-animated shading rig in the next section.

\subsection{Results}

In this section we compare continuous shadow animation, and dependence on mesh density, with Todo et al. 2007 [3], and shape interpolation performance with Nader and Guennebaud [4]. We also show qualitative examples of how our shading rig model supports the use-cases introduced below.

\subsubsection{Examples and Use-Cases}

We first show how our shading rig approach can achieve common use cases for shade editing using our parametric edits.

\section{Facial Expression and Detail}

In hand-drawn shading, facial expression and detail are exaggerated with stylised shadows. In Figure 4.9 we show examples of hand-drawn shading for facial expression and detail, as well as how our edits can serve this purpose. In this example, we use rounded shadow edits to darken the eyes as the "deep pools of shadow" [138] mentioned in Section 4.3. Curved and sharpened edits exaggerate brow wrinkles and cheekbones such as shown in the hand-drawn examples. 


\section{Muscle Shading}

We show how muscle lines can be introduced with the shading rig. Shadows added to emphasise crease details such as muscles are referred to as "inner lines" $[102,103]$. Figure 4.10 shows hand-drawn muscle shading, and how our shading edits can model these shadows as parametric inner lines. This use-case of cartoon muscle lines, typically solved using textures or mesh editing, can now achieve vector quality while remaining animatable with our approach.

\section{Rembrandt Triangle}

The Rembrandt triangle $[17,141]$ is a realistic lighting phenomenon, ubiquitous in both stylised shading and cinematic lighting. It occurs when an elongated nose
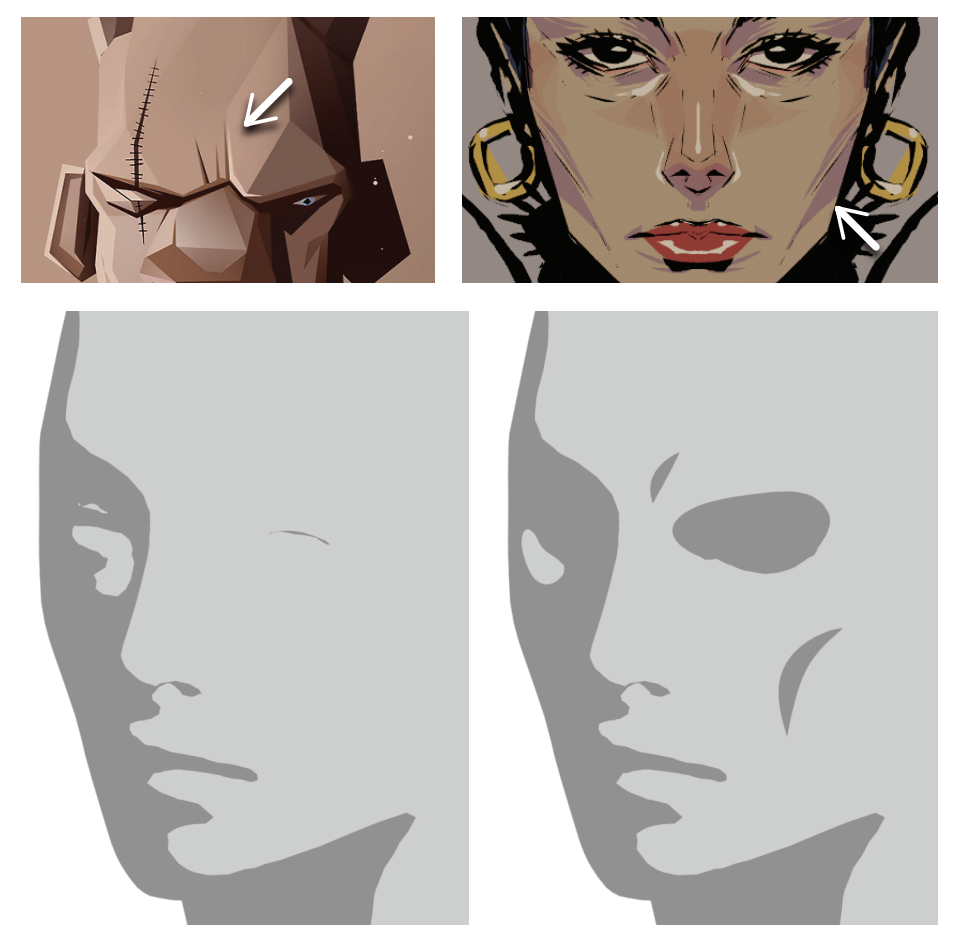

Figure 4.9: Top: Example hand-drawn shadows used for facial expression and detail. Bottom: unedited toon shading (left) and edited expression using shading edits (right). This result required 5 edits. Creature concept: CWildboy Studios used with permission. Female artwork by Claudio Grassi licensed under CC BY. Head model by Vinicius Nunes licensed under CC BY. 

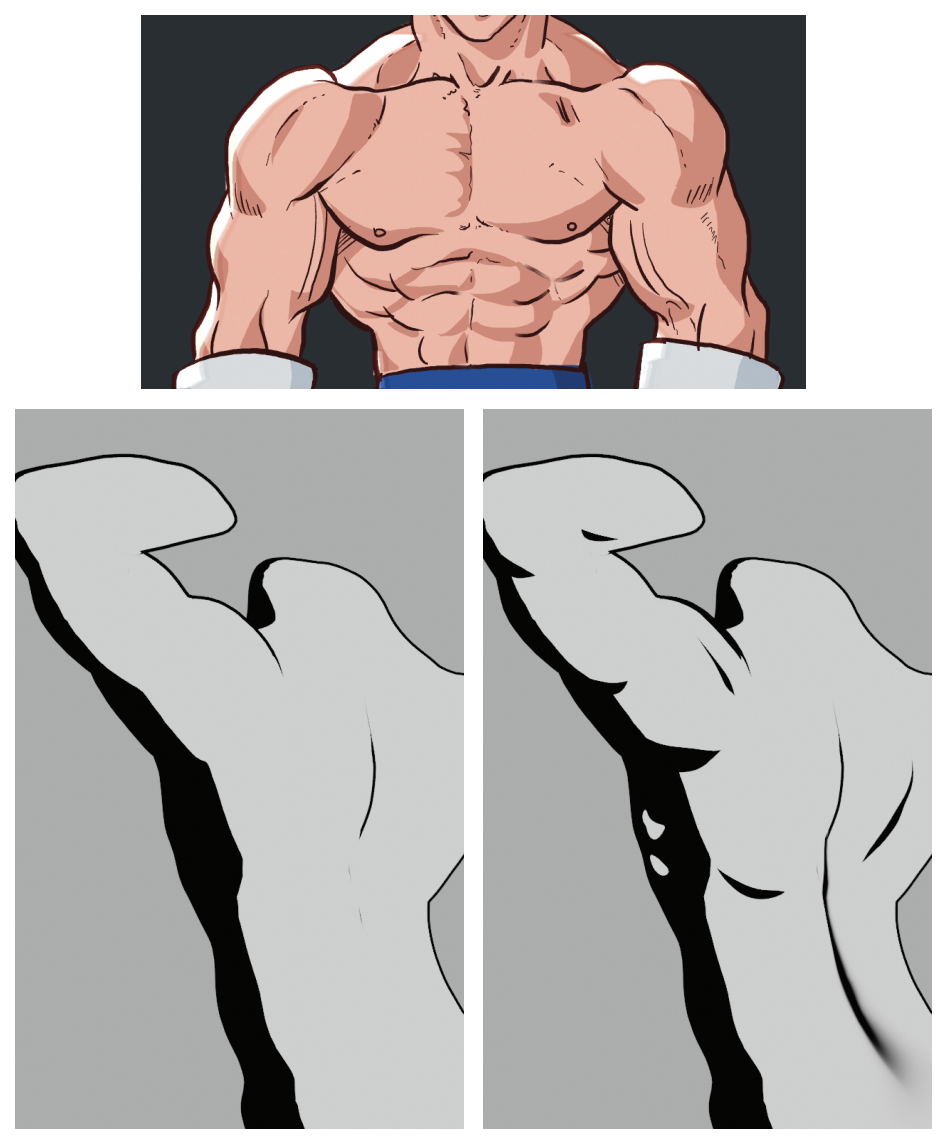

Figure 4.10: Top: hand-drawn stylised muscle shading. Bottom: unedited toon shading (left) and added muscle shading using 10 shading edits (right). Artwork by Brain Graft licensed under CC BY. Model by Julien Kaspar licensed under CC BY.

shadow meets the cheek shadow creating a triangular lit area beneath the eye. Although our edit parameters were not specifically designed for triangles, we show an example of a similar shape, in Figure 4.11. This required low Anisotropy, high Sharpness, increased Bulge to weigh one side, and increased Bend to curve up around the eye. The edits were added directly, even though the unedited result did not produce a nose shadow. 

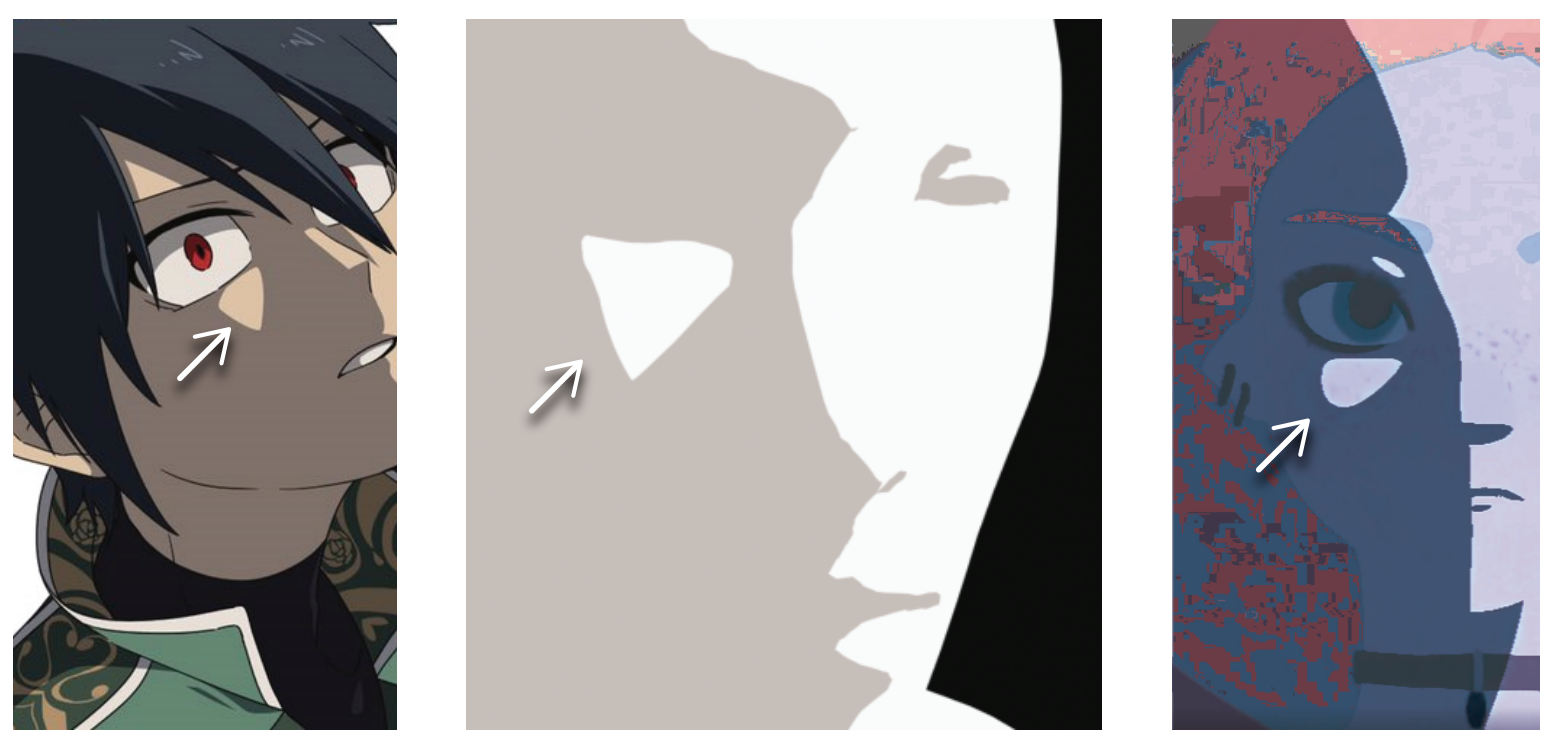

Figure 4.11: Left: Hand-drawn stylised shading with Rembrandt triangle under the eye. Middle: Edited toon shading with the shading rig, applying a Rembrandt triangle without a nose shadow being cast. A single intensity edit is used to blend with existing key lighting. Right: rounded Rembrandt triangle added also using one mask edit with high Normal Smoothing. Character artwork: CRodu used with permission.

\section{Local Detail Control}

We show that even on high detail surface meshes, our edits can control shadows by varying the Normal Smoothing parameter on each edit. In the example in Figure 4.12 , surface details on the snake are revealed only on the tail. The face is left abstract to only show face details like the eyes and mouth. We achieve this with different edits having their own Normal Smooth value. The underside of the snake also employs local softening as defined by the artist.

\section{Deformation Support}

We also show our method supports animated and deforming characters, using our deformation tracking function. Artists can design edits at a single undeformed pose, and have their edits automatically track surface deformations. In Figure 4.13 an edit is placed near the left corner of the mouth. Without any further manual animation 

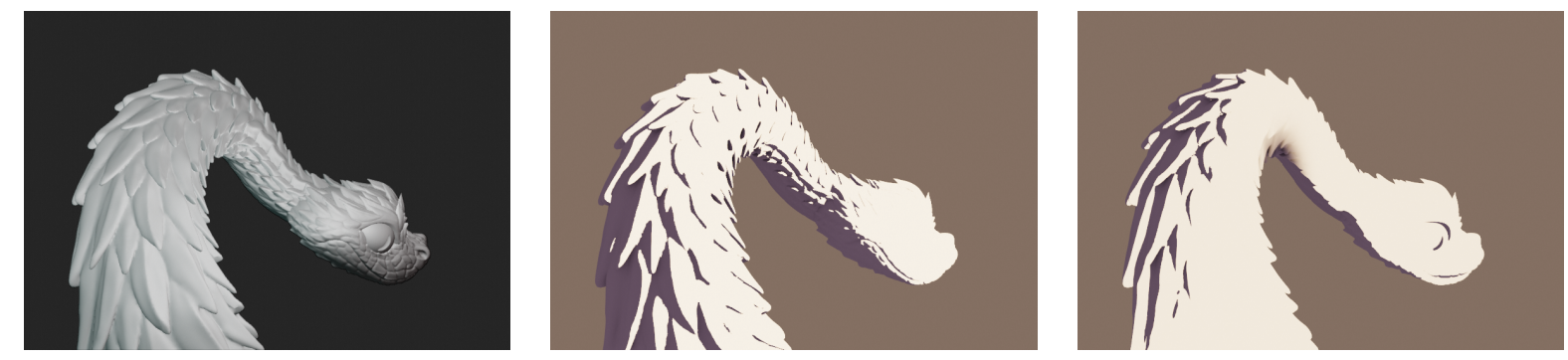

Figure 4.12: Left: high detail mesh. Middle: base toon shading. Right: edited result (9 edits). Model by Julien Kaspar licensed under CC BY.

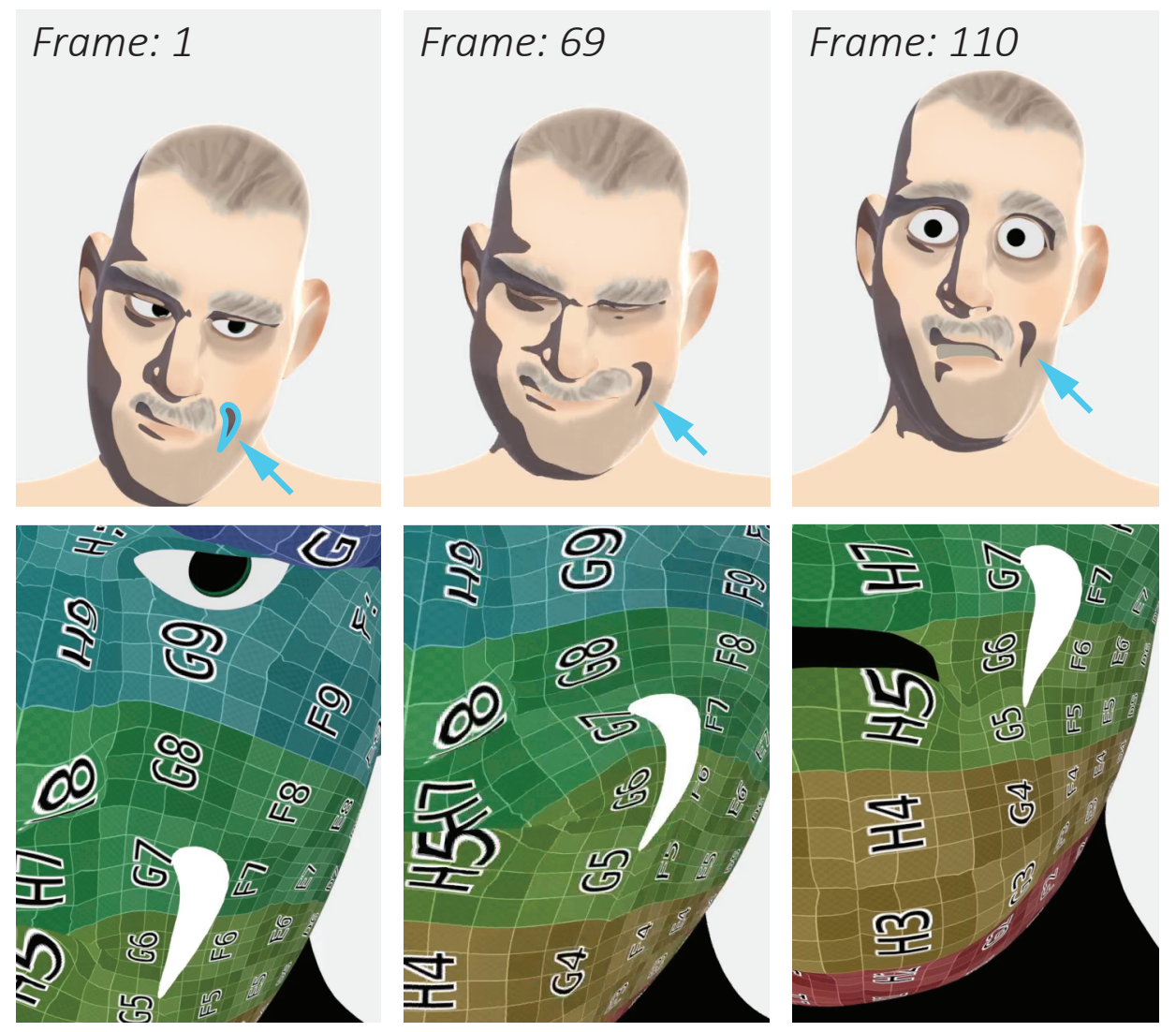

Figure 4.13: Top: Edit made on the left-most frame (outlined in blue) can track mesh deformation throughout an animation sequence. Bottom: edit shown in white precisely tracks mesh surface parametrisation and remains animatable and mesh/texture resolution independent. Model by Daniel Martinez Lara licensed under CC BY. 
of this edit, it tracks the surface mesh during deformation. We also visualise the edit with the surface's UV parametrisation showing precise correspondence. This matches the behaviour of other techniques, while remaining animatable at vector quality.
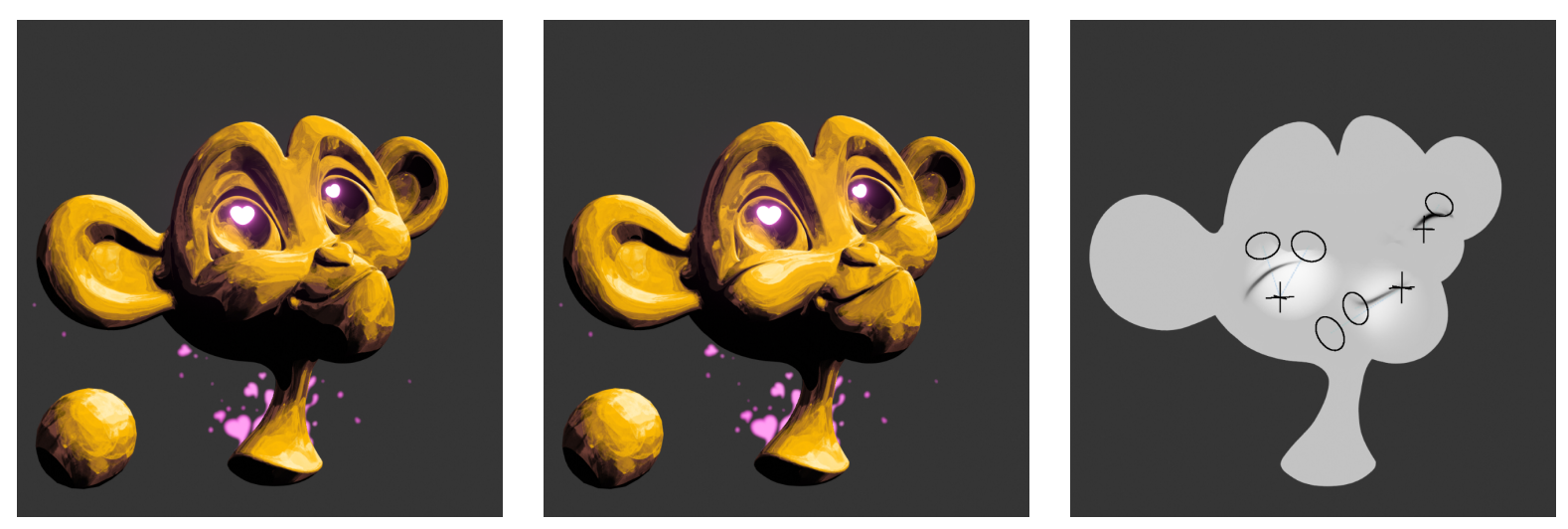

Figure 4.14: Left: Lit-sphere shading [2]. Middle: Edited shading using shading rig (5 edits). Right: Shading Rig (circle and axis controllers) and its resulting intensity field. Model by Julien Kaspar licensed under CC BY.

\section{Other styles}

Our shading rig approach can be used outside two-tone toon shading. In Figure 4.14 we show how our Intensity Edits are suitable for editing artist-defined shading gradation such as in lit-sphere shading $[142,2]$. We achieve this by locally scaling the lit-sphere texture of [2] based on the edited intensity field generated from the shading rig.

\subsubsection{Shadow animation with lighting changes}

After pre-animating, the shading rig smoothly transitions between the keyframes as the key light is rotated. This has the effect of shading edits moving in space, and morphing in shape, to dynamically correct the toon shading result as desired by the artist. We show an example result in Figure 4.15. For comparison, we show the 

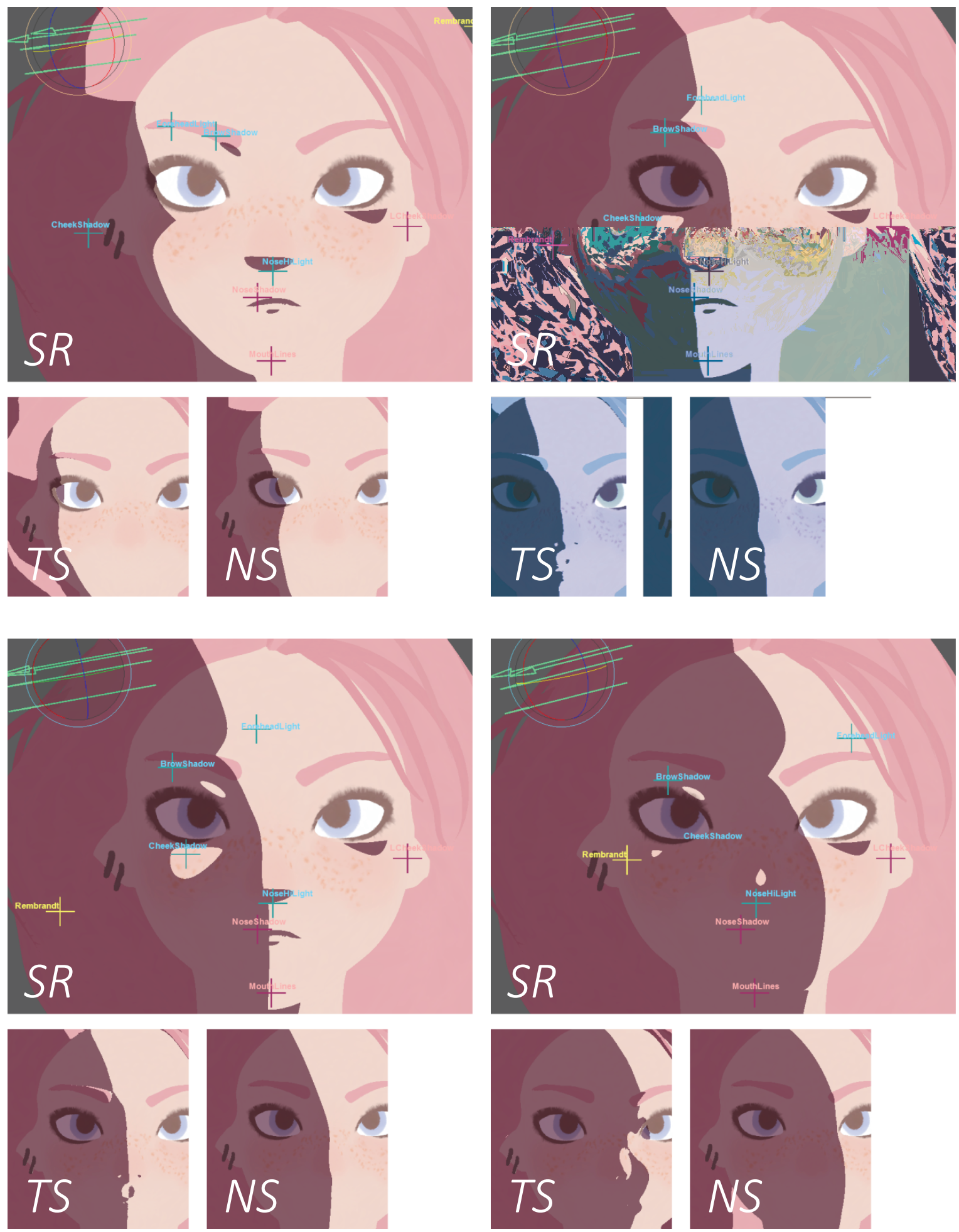

Figure 4.15: Top row (SR): Shadow animation produced by a pre-animated shading rig (collection of cross-shaped locators) as the key light is rotated. Below each large frame: conventional toon shading (TS) and normal smoothed toon shading (NS) to be edited. 
unedited toon shading result and the normal smoothed result on which the shading rig applied edits.

This example was animated with four dynamic edits, each using 2-4 positional keyframes and 1-3 parameter keyframes. To make edits smoothly appear and disappear, their positions were moved further and closer to the surface mesh. This gives simple control over the size of the edit's shape just like conventional point lights with a distance based intensity falloff.

Note the intentionally added stylised Rembrandt triangle which the unedited result did not produce. This edit was added directly, regardless of the geometry and conventional lighting. This edit's shape was also animated to emerge rounded, and then sharpened at the right light angles, using the Sharpness and Anisotropy parameters. From this we can see the level of control artists have over smooth stylised shadow animation, using the shading rig.

We note that this level of control for dynamic shadow design is crucial to avoid undesirable shadows from realistic lighting. In this case especially, long nose shadows are required to produce Rembrandt lighting in reality [141], but are considered undesirable [20] before the Rembrandt triangle is made. With the shading rig, we can smoothly animate the triangle emerging while keeping the nose shadow short.

The edit at the forehead is also used to exaggerate the browline by sharply pinching the shading boundary at the brow. This is animated from left to right with the key light, to maintain this contour shape while the key light is rotated. Just three keyframes were need for this highlight using the shading rig.

In Figure 4.16 we show how this animation can be applied to a dynamic character moving relative to a key light. This example, implemented in Unreal Engine 4, shows the artist-defined shadow behavior being reproduced as the character turns their head. This result uses the method $\mathrm{d}$ in Section 4.4.2. Please see the supplementary video for the full animation. 

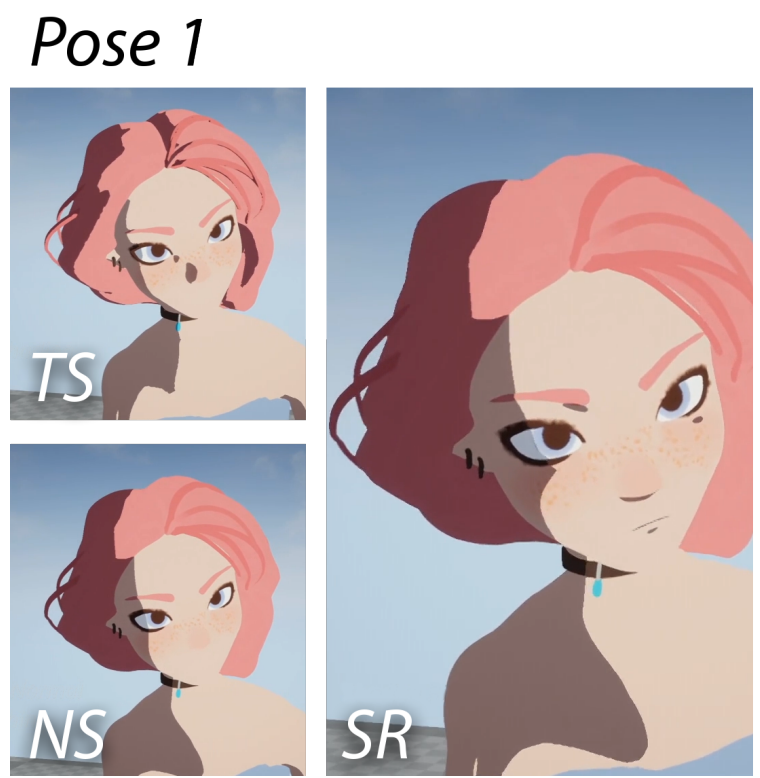

\section{Pose 2}
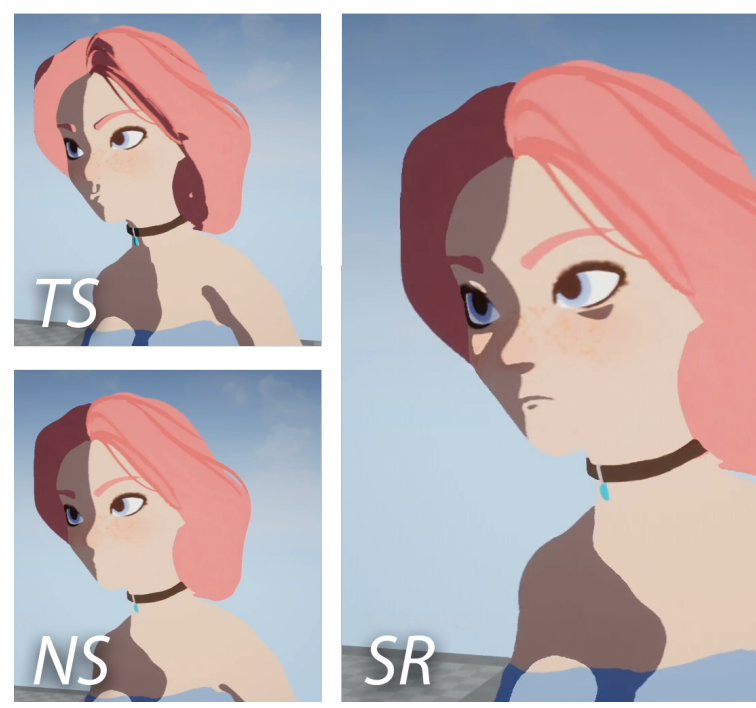

Figure 4.16: Shading rig animation result, used for a dynamic character moving relative to key lighting. TS: toon shading, NS: normal smoothing, SR: shading rig.

\subsubsection{Comparison With Previous Work}

\section{Smooth animation}

We expect shape interpolation between two keyframes to achieve continuous motion and shape transformation, without gaps or doubling, as described by Bonneel et al. [133]. While offset painting [3] supports arbitrary shade modification, edits cannot be moved across the surface without redrawing the shape. Our method requires no redrawing of designed shapes and are simply moved across the surface mesh like a light source. Furthermore, we achieve continuous shade animation with limited keyframes, as shown in Figure 4.17. Here we see the edits of Todo et al. 2007 [3] disappear and reappear between keyframes, due to linear blending between the fixed intensity distributions of their edits. Our approach of parametrising each edit achieves true distribution interpolation, preserving continuous motion and shape interpolation between keyframes. This more clearly illustrated in Figure 3 of the Appendix. 


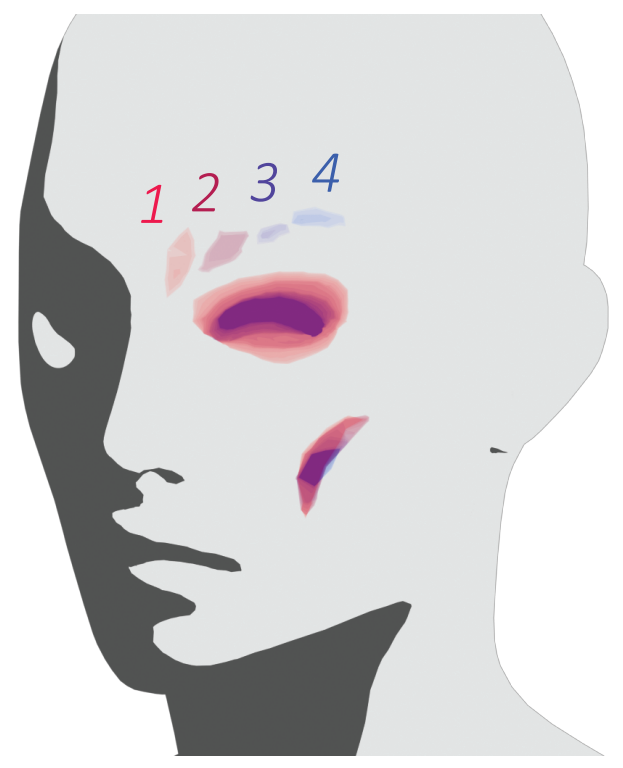

(a) Todo et al. 2007 [3]

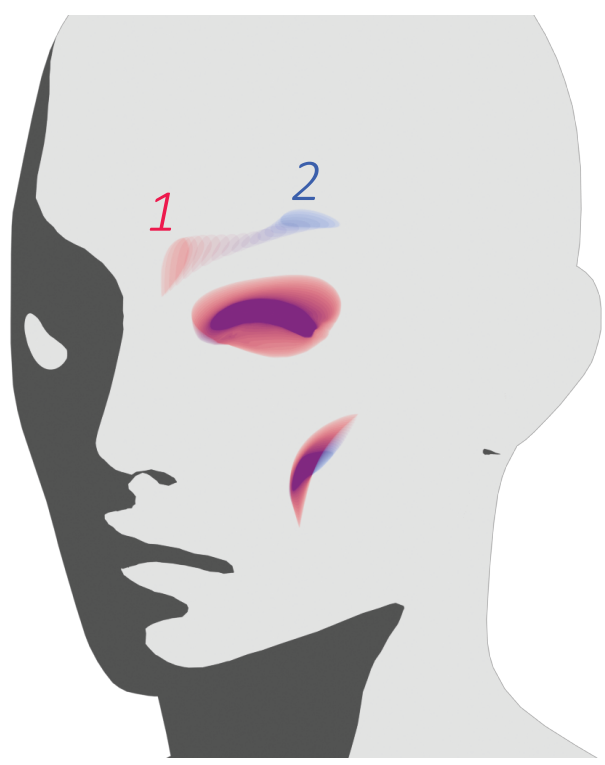

(b) Our approach

Figure 4.17: Example animated shade edit comparison with colour variation from red (start) to blue (end). (a) Offset painting (Todo et al. 2007 [3]) result with 4 keyframes (numbered), showing fragmented motion of shadow. (b) our approach requires only 2 keyframes to yield continuous motion and shape manipulation. 


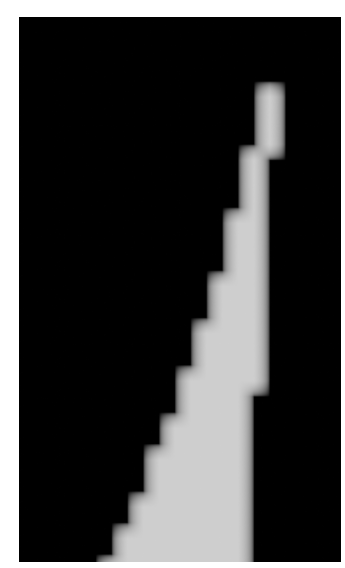

(a)

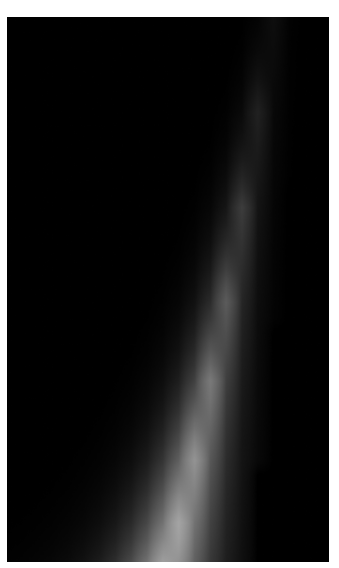

(b)

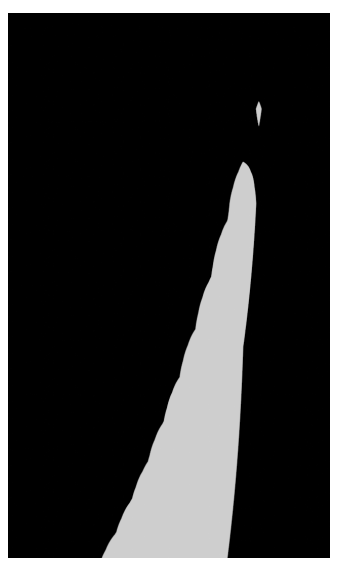

(c)

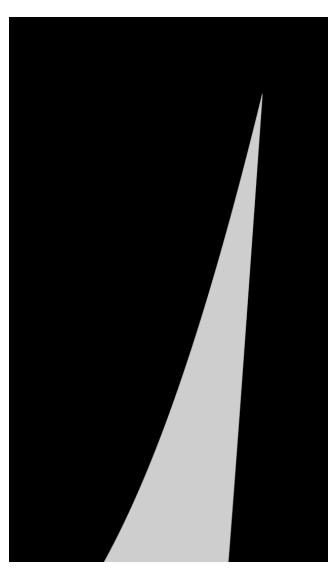

(d)

Figure 4.18: Texture mapping using a rasterised shading texture exhibits pixelation artifacts upon magnification (a). Using a rasterised intensity distribution (b) for optimal transport [4] causes artifacts after thresholding (c). Our parametric distribution (d) provides artifact free reproduction of sharp cusps and shape boundary.

\section{Rasterisation Artifact Comparison}

Bonneel et al. [133] proposed to use optimal mass transport for continuous distribution interpolation that specifically solves the problem shown in Figure 4.17 and Figure 3 of the Appendix. The method of Nader and Guennebaud [4] achieves this in real-time on an image grid. Although suitable for fast texture mapping on GPUs, this would exhibit artifacts upon close-up. In Figure 4.18, we compare these artifacts and show that our approach provides artifact free reproduction of sharp edits and shape boundary. The boundary of our edit shapes can be antialiased using screen-space derivatives as done by Loop and Blinn [128].

While using grid-free mass transport techniques would avoid pixelation artifacts, other sampling issues would arise. The grid-free method of Bonneel et al. [133] use RBFs to approximate shapes, but this cannot reproduce sharp cusps as mentioned in the related work. Representing distributions as weighted point clouds using weighted Dirac delta functions [143] would produce aliasing artifacts as demonstrated by Bonneel et al. [133]. 


\section{Performance Comparison With Image Interpolation}

While the above artifacts can be reduced with higher texture resolution, we show that our 2D shape interpolation has a much lower computational cost than that of Nader and Guennebaud [4]. As mentioned in Section 4.2, the pre-computation time demanded by their method would become prohibitive for editing with high resolution images. Our method requires no pre-computation, allowing for artists to animate shading with instant feedback.We also compare the performance cost of animation provided by each method at runtime.

The 2D shape interpolation of our shading rig model and the method of Nader and Guennebaud [4] can both be parallelised on the GPU. To compare the total number of operations we measure running times using single-threaded, non-parallelised, CPU implementations of each method. We used an Intel Xeon W-2123 3.60 GHz, with 16 GB of RAM. Both methods interpolate parameters representing the output 2D shape, with negligible running time. However, we find that the rasterisation step of Nader and Guennebaud [4] is much slower than ours. Figure 4.19 shows running times. We can see that our shape interpolation is an order of magnitude faster. Thus artists can use more dynamic shading edits with our approach than with mass transport, for interactive applications. We note that shape interpolation based on mass transport still permits arbitrarily shaped shading edits.

After implementing our method as a GPU shader, we observed real-time rendering and animation of more complex shading rigs (tens of edits) in both Maya and Blender. We observed real-time performance for thousands of edits (more than necessary for practical use), in a stylised game environment, in Unreal Engine 4. We give further performance tests in the Appendix.

\section{Mesh Density Independence}

In Figure 4.20, we show that edited shading shapes degrade after mesh decimation, when relying on vertex normal edits, or offsets painted as vertex colours [3]. Our parametric edits are seen to preserve their shape regardless of mesh density and topology, providing greater freedom of expression to artists. In particular the 


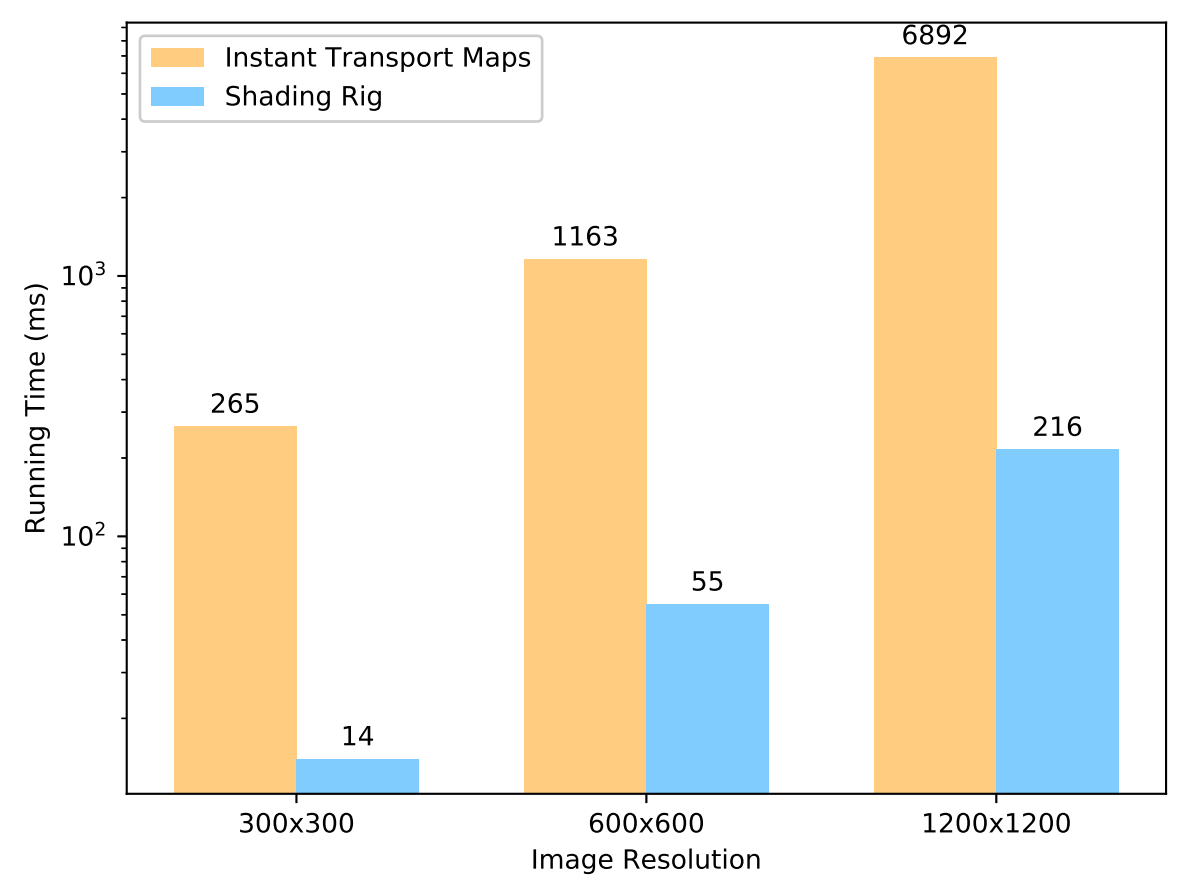

Figure 4.19: Shape interpolation running time comparison (log scale) between Instant Transport Maps [4] and our model, using non-parallelised CPU implementations of each method to compare number of operations.

shadow shapes permitted by mesh-based edits are severely limited by mesh topology.

Art-directed Toon Compositing: In the previous chapter we art-direct toon shading based on background lighting conditions (Figure 3.14). The shading rig can be combined with the EM latent space to art-direct shading when environment lighting rotates. We show edited toon shading in real-world environment lighting in Figure 4.21. We assign the dominant light (DL) direction to the key-light direction. Colours from the environment map (EM) are sampled by using a shader that directs smoothed surface normals toward and away from the DL, in the light and shadow regions respectively. This provides regions of relatively flat colours to mimic two-tone shading while still reflecting colours from the EM. 

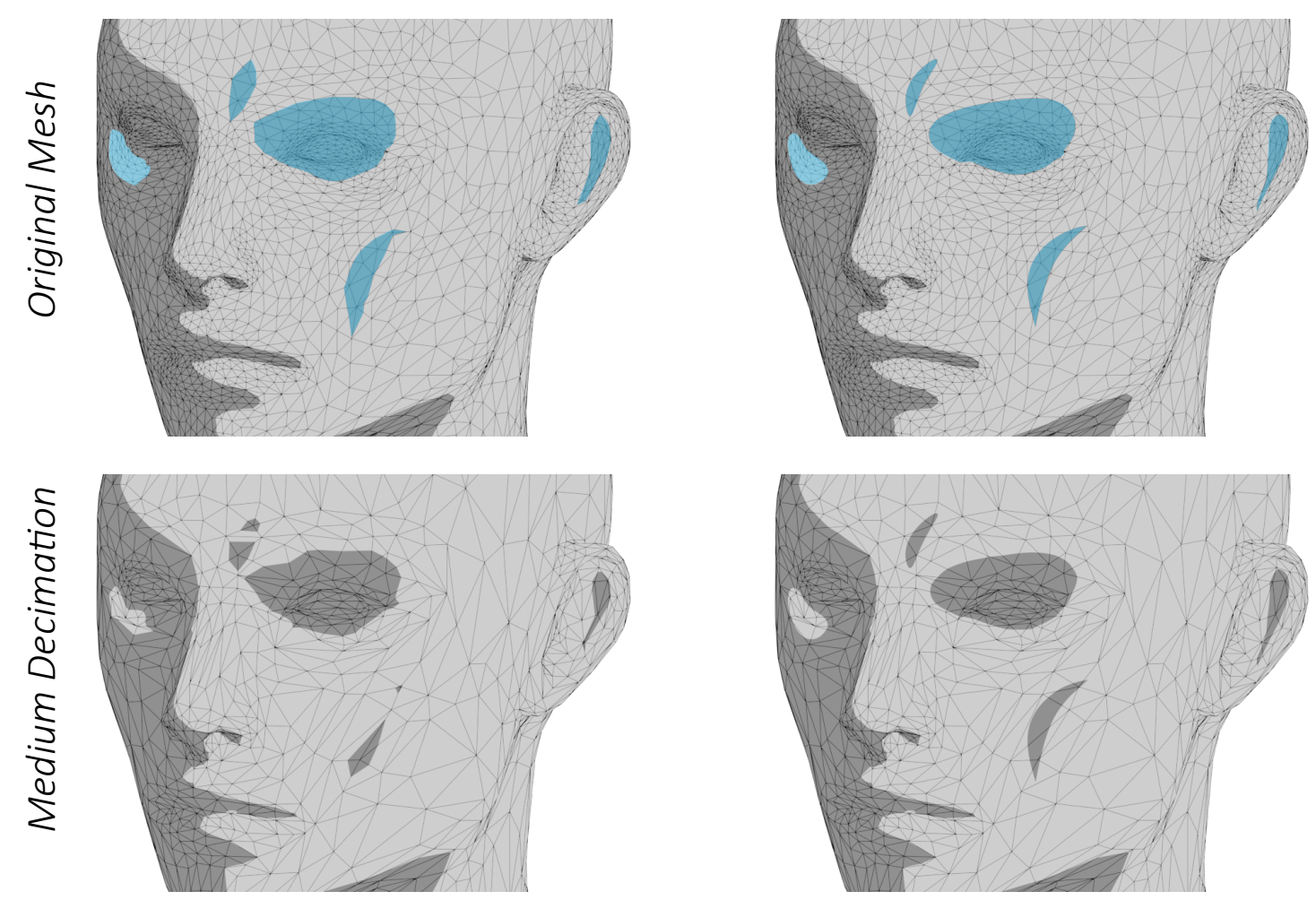

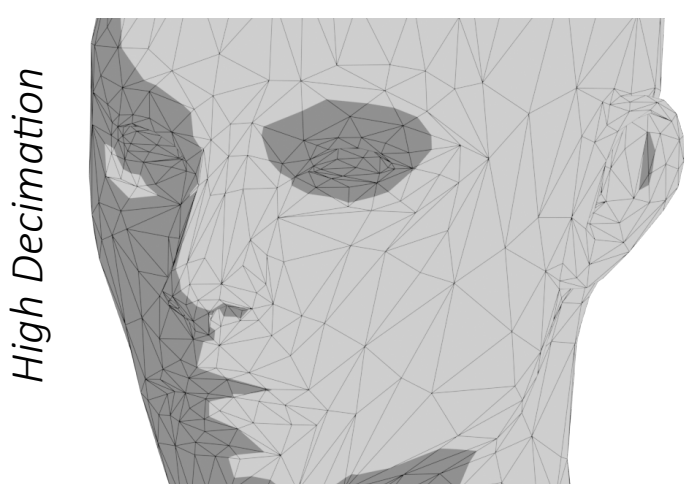

(a) Todo et al. 2007 [3]

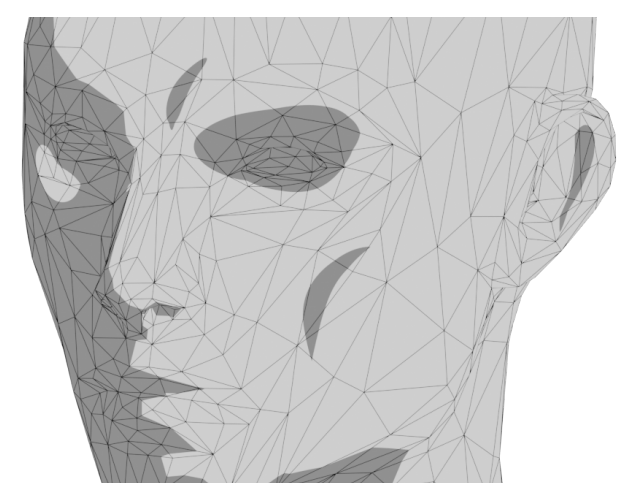

(b) Our approach

Figure 4.20: Original shade edits made on a surface mesh (blue) and their dependence on mesh density. a) Limitation of Todo et al. 2007 [3] where edit shapes are affected by mesh density and topology. b) Our parametric edits preserve their shape regardless of mesh resolution and topology. 

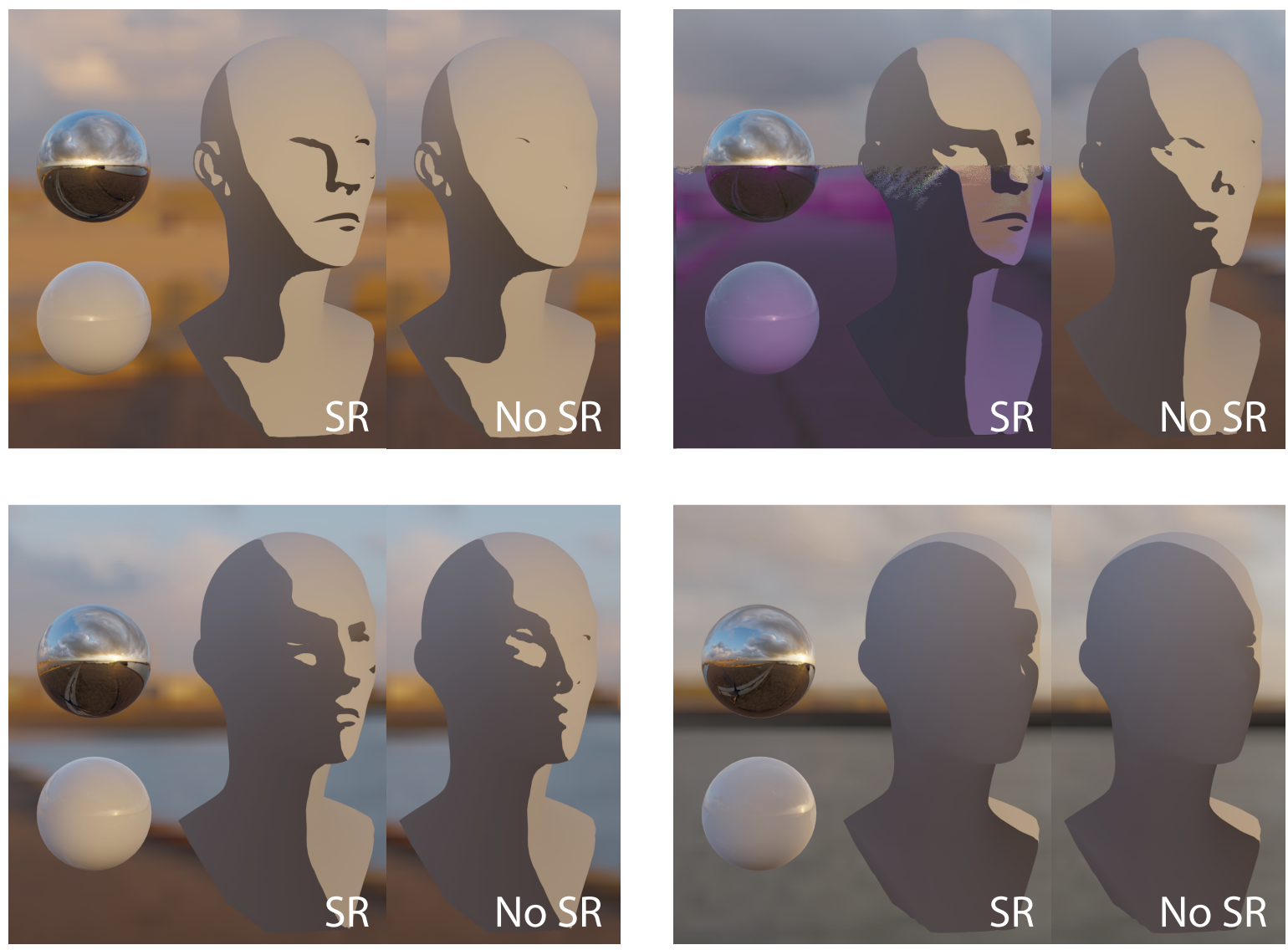

Figure 4.21: For the task of compositing cartoons into real-world backgrounds, the shading rig can be applied to environmental lighting. We show toon shading variation with EM rotation, with the shading rig applied (SR) and without (No SR). 


\subsubsection{Artist Feedback}

We implemented the Shading Rig prototype interface as a Maya plugin. We showed this to several artists to assess the potential of industry usage and compatibility of our method. In our prototype, the artists could freely edit toon shading on a stylised model with our method. We received feedback from a technical artist from a Japanese animation company producing world-renowned 2D cel animation and 3D cartoon films. We also consulted 3 professional 3D artists and 7 graduate students in 3D animation and visual effects. We gave all the artists the task of using our prototype to match the reference animation shown in Figure 4.15. However, they could design different animations to test other aspects based on their own artistic requirements. All artists were proficient with animation in Maya. In the appendix, we provide the prompts given to the artists to provide feedback. However, there was no obligation to answer them directly and they were free to give other feedback and candid responses.

\section{Industry Technical Artist}

The 3D technical artist from the animation company had expertise in 3D toon shading and editing practices. He commented "Wow! It looks cool, this could be an alternate way to relight toon shaded characters". Regarding industry adoption he commented "Using this tool shadows and highlight areas can be edited before rendering of the shots. Thus eliminating the need for editing the light and shadow areas in post using techniques such as rotoscopy". This verifies our intended use case of pre-animating the shading rig for directed shading without artist intervention. Regarding the visual results of the tool, he confirmed our model produces a sufficient range of shapes for toon shade editing tasks, and said "the shading rig produces simplistic shape results which are pleasing to the eye". One interesting concern he raised was that "To achieve the desired result the shading rig needs to be animated. Which is not the specialisation of a lighting artist so it may produce confusion in delegation of work". 


\section{Professional 3D artists}

One 3D artist could see potential in designing "3D character model sheets" with our method, expressing that the shading rig's ability to "propagate shading rig changes through all shots at once" would be very useful. This artist also mentioned it was "fun because it gives fast exploration, with a strong, continuous, instant feedback loop", and that artists "can get started with editing immediately during 3D lighting stage, instead of waiting for clean-up at post-production".

Other artists said our edits were "easy to control" and "pretty intuitive". They were able to shape and animate edits within minutes. Regarding the visual results, they confirmed the edit shapes looked natural and that our approach "can generate really cool effects".

\section{Graduate 3D students}

The students had between 5-8 years prior 3D industry experience. One reported our prototype as being "easier to gain a similar appearance" to a reference example. Others commented that it "gives us many more chances to make details" and gave remarks such as "Fun stuff! Really influences emotional expression".

Some students expressed that controlling and animating our parameters can be tedious and requires some time to learn. However, given our results showing shading animation with fewer keyframes and no pre-computation time, the shading rig can save asset pre-production time.

\subsection{Limitations and Future Work}

We addressed the problem of dynamic stylised shading edits by parameterising individual edits. Our novel parametric model produce local stylised shade editing shapes. This allows for smooth animation while maintaining vector quality and resolving sharp cusps upon close-ups. Based on artistic shading literature, our edits are designed to produce expressive shapes to support common editing practices and adding intentional details. 
We have shown smoother animation results compared to the method of Todo et al. 2007 [3], with fewer keyframes. We also demonstrated that our 2D shape and intensity distribution interpolation incurs dramatically lower computational load compared to real-time optimal transport [4].

Artists can build a shading rig to edit the result of conventional toon shading. Our example pre-animated shading rig achieves real-time art-directed toon shading without artist intervention.

\section{Limitations}

Our parametric edit model can produce simple, round shapes with optional sharp points, suitable for characters with organic forms. However, for mechanical objects such as robots, weapons, and cars, our parameters may have limited support for producing more complex edits for these geometric surfaces. Similarly, our model may also have limited support for producing fine-scale hair details and shade editing on complex large-scale background environments.

Our approach also demands a time investment to pre-animate the shading rig with all lighting changes. Animated shading rig results produced in this paper took up to several hours to create in our prototype. We note, however, that 3D character preparation using existing editing techniques for stylised games, has been reported to take months [102]. Considering this, and our improved keyframing results, our approach will reduce preparation time while enabling smooth shade animation at vector quality.

\section{Future Work}

Compared to our approach, vector mask manipulation from rotoscopy remains the most flexible in designing arbitrary shadow animation. Further research into rendering real-time, animatable, vector masks on 3D surfaces would be valuable. While interfaces for 3D lighting tasks have been evaluated [144], further interface development with artists, followed by user studies could help to assess what user interfaces are best suited to stylised shade editing. 


\section{Chapter 5}

\section{Conclusion}

The field of computer graphics is abundant in research efforts to improve accuracy in photorealistic rendering. Unfortunately for story-driven media such as films and games, artists are seldom satisfied by realistic rendering, and need to manually art-direct the results. The goals of this thesis were to achieve automatic preservation of art direction in real scenes. Since realistic rendering is one component of art-directed 3D digital media, we began with a user study in Chapter 2 to inform perceptual optimisation of realistic compositing. For art-directed composition, Chapters 3 and 4 presented new techniques to preserve stylistic edits to appearance, lighting, and shadows. Current research in art-directable and stylised rendering can support artistic editing for one scene, but it remains tedious for artists to manually edit appearance, and impossible for live games and AR/MR applications. We have shown our methods support preserving art direction in dynamic lighting environments.

\subsection{Achieved Goals}

We relate the achievements of this thesis to the goals set in the introduction, in the following summary of contributions:

- In Chapter 2, we achieved the first goal which aimed to optimise the cost of 
real-world scene capture. We measured how background scene fidelity impacts perceived seamlessness, and determined perceptual thresholds for several compositing scenarios. Our findings show that depth-capture resolution can be reduced in certain cases. This can be used to speed up existing depth estimation or scanning of the background, for compositing virtual foreground objects.

- Chapter 3 achieved the second goal of preserving art direction across different real-world backgrounds. We encode real-world lighting environments into a 2D latent space. Our 2D embedding of EMs expressed a consistent semantically meaningful organisation of various EMs. Using this space, artists can verify the appearance of an object under different lighting conditions, and make the necessary adjustments to meet art direction across all background lighting conditions. Artists can edit appearance on the latent space, then the virtual object can automatically exhibit the desired appearance intended for that background. This solves the issue of artists compromising on their desired 3D object appearance for all backgrounds.

- Chapter 4 achieved the third goal of automatically preserving art-directed shadows under a dynamic light direction. Chapter 3 dealt with fixed rotation of the background environment, requiring further work to handle changing light angles. We distilled art-directed shadows into a parametric shape model for shadow editing. Several edits form a shading rig for artists to control and animate shadows as the lighting angle changes. This enables further art direction than what was possible with just the EM latent space, which assumed a fixed horizontal light angle.

\subsection{Limitations}

The above contributions provide novel solutions to the described problems, but are limited in the following ways. The user study in Chapter 2 measured the perceptually optimal depth resolution for seamless compositing. However, we proposed no solution to detect which of the four scenarios is used in an image. 
Being able to detect which perceptual threshold to use in an image would allow full automation for optimising depth capture or estimation in a composite. One could also extend this to detecting multiple perceptual thresholds for different regions in an image, to further optimise depth acquisition.

EM latent space and shading rig deal with global lighting. Lighting in EMs are considered infinitely far from the object. The key-light direction in the shading rig is also considered a directional light, not a positional light. In the case of the EM latent space, we only encode light arriving at one point. In reality, light coming from different directions and positions varies spatially across the object, and is not encoded in our latent space. For the shading rig, it is possible to animate shading edits with respect to the position of a key point light. However, we imagine the task of manually animating the shading rig with respect to multiple lights would be impractical, requiring extension of our solution.

For the art direction solutions presented in this thesis we require manual editing during pre-production, to save repeated work done in post-production. This may seem like a limitation as this step is not automated. However, end-to-end automation limits expressive freedom to existing known styles and assumes artists' aesthetic intent. The solutions in this thesis instead sought the freedom for artists to create novel styles, and self-evaluate the results based on their own aesthetic requirements.

\subsection{Future Work}

This thesis discussed art-directed compositing and proposed techniques through which artists can control stylisation based on changing real-world lighting environments, and light angles. Besides the future work given for each chapter, one future research direction would be in compositing photorealistic content into stylised backgrounds. This has been researched by Luan et al. [145] who style the inserted object to match that of the background artwork. However, they only match texture and colour. An unexplored alternative could be to estimate lighting from the artwork to stylistically re-light the composited object. 
Another research direction could be evaluating perceived seamlessness of compositing stylised characters into real scenes. Chapter 2 measured seamlessness as a user's belief that a composited object originally existing in the background scene. One could redefine seamlessness to mean that stylisation of realistic lighting matches a real-background. Previously Bousseau et al. [146] measured stylised gloss perception by asking users to match realistic and stylised glossy material renderings. A similar experiment could measure how well users can match realistic EM lighting to a corresponding stylised rendering. The EM latent space could then be used as a selection tool for this task. This result would inform how stylisation perceptually affects "seamless" blending of non-photorealistic styles into real scenes. 


\section{Appendix A}

\section{Shading Rig Appendix}

\section{A.1 Local Texture Space}

Our local texture space was designed to achieve the fill light behaviour [18] described in Section 3. We considered the method of Todo et al. 2013 [2] which allows artists to define shading on a lit-sphere texture. This has the benefit of lit-sphere edits automatically moving with a changing light direction. However, editing a lit-sphere texture will affect other surface points that share the same normals as the selected region (see Figure A.1). Instead, we need to able to select a local area on the surface to be edited.

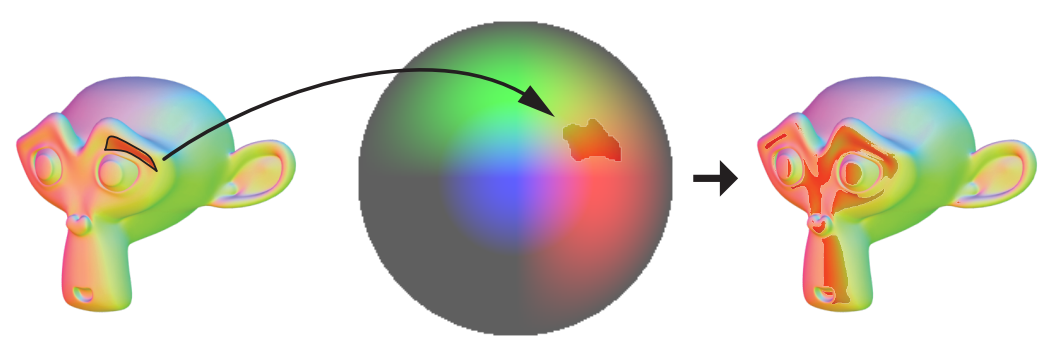

Figure A.1: Limitation of lit-sphere approach [2] for artistic editing. Left: Artistspecified local area on 3D model where shading must be edited. Middle: corresponding area on lit-sphere domain where the stylisation texture would be modified. Right: affected area when applying the edited lit-sphere texture is far greater than the specified region. 
While 3D software provide texture projection interfaces for local decal placement [147], we implement a simple texture space behaving similar to point lighting. Specifically, this allows our parametric distribution (Section 4.1) to exhibit light intensity falloff based on distance, to match the fill light paradigm. Our projection uses the light space $\left[\mathbf{l}_{\mathbf{x}} \mathbf{l}_{\mathbf{y}} \mathbf{l}_{\mathbf{z}}\right]$ and light space normal $\mathbf{n}_{\mathbf{l}}$ of Todo et al. 2013 [2], modified to be spatially varying. Since our texture space does not depend on the surface normal, we redefine the light space normal $\mathbf{n}_{\mathbf{l}}$ as the light space vector $\mathbf{v}_{\mathbf{l}}$.

As mentioned, $p_{1}$ is the edit's location in 3D space, and the texture space is projected toward the light origin point $\mathbf{p}_{\mathbf{o}}$. This direction is denoted by $\mathbf{l}_{z}$ and its corresponding light space basis vectors as $\mathbf{l}_{x}$ and $\mathbf{l}_{y}$. Given $\mathbf{l}_{z}$, we form an orthonormal basis such that $\mathbf{l}_{x}$ is orthogonal to $\mathbf{v}_{\mathbf{u p}}$ (the up direction vector in world space).

The light space vector is defined as $\mathbf{v}_{\mathbf{l}}=\left(v_{l x}, v_{l y}, v_{l z}\right)=\left(\mathbf{v} \cdot \mathbf{l}_{\mathbf{x}}, \mathbf{v} \cdot \mathbf{l}_{\mathbf{y}}, \mathbf{v} \cdot \mathbf{l}_{\mathbf{z}}\right)$. where $\mathbf{v}$ is the direction from the world space position of the surface shading point $p_{w}$, to $p_{l}$.

These parameters, the light space vectors, and relevant surface information, are shown in Figure A.2. The texture space is then defined as $(u, v)=(r \cos \theta, r \sin \theta)$ where $r=\cos ^{-1}\left(v_{l z}\right)$ and $\theta=\tan ^{-1}\left(v_{l y} / v_{l x}\right)$.

We note that this simple projection cannot handle repeated and high curvature. The edit shapes would be repeated on the behind surface that curves away from the point source. Discrete Exponential Maps [147] would solve this issue, but is dependent on mesh topology and becomes slow for large projections over many triangles. In our case, the Normal Smooth $(\tau)$ or Radius $(R)$ parameters should be reduced to mitigate the issue.

\section{A.2 Symbols and Notation}

Table A.1 includes all symbols and notation used in Section 4 and Appendix A.1. 


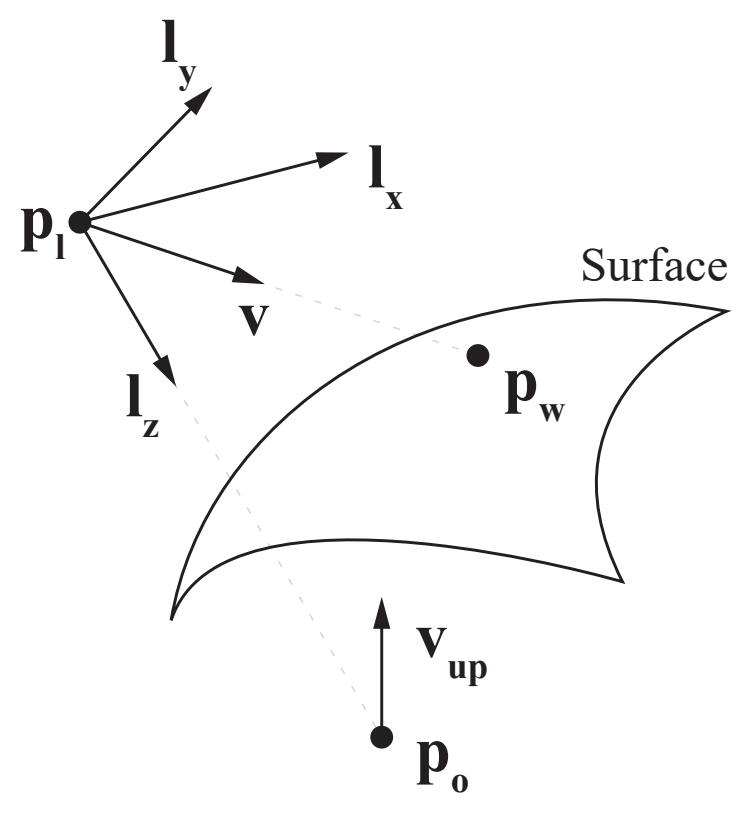

Figure A.2: Light space vectors computed with respect to the light position $\mathrm{p}_{1}$ and light origin $\mathbf{p}_{\mathrm{o}}$.

\section{A.3 Shading Interpolation Result}

Figure A.3 shows how our parametric approach achieves true distribution interpolation. This preserving continuous shading interpolation between keyframes, compared to the method of Todo et al. 2007 [3].

\section{A.4 Performance Evaluation}

For the RBF-based animation system, we measure the worst-case performance costs for keyframing and real-time evaluation, as the number of keyframes increase. In Figure A.4, we measure the RBF solve time as more keyframes are added for all parameters of a single edit. We see that adding a keyframe is fast for a single edit, even up to 200 keyframes. Figure A.5 shows the RBF evaluation time for all parameters in a shading rig with 10 and 100 edits. Figure A.6 shows GPU rendering time as the number of edits increase. In Unreal Engine 4, we render the 
GPU shader for different screen percentages of the total screen resolution of $1920 \times 1080$. The screen percentage is how much area a stylised character takes up of the viewport.

The timings were measured using an Intel Xeon W-2123 3.60 GHz CPU, on 16 GB of RAM, and a GeForce GTX 1080 GPU.

The real-time evaluation result shows that our shading rig achieves real-time performance. While we expect that artists will only use tens of keyframes per edit, and tens of dynamic edits per character, testing at these larger ranges still show real-time rendering with multiple characters can be achieved.

Not all edits and parameters need to be animated, so these performance results show the worst-case performance cost in our unoptimised implementation. In practice, shading rigs for off-screen and distant characters need not be updated. In real-time games and previsualisation, the shading rig can be updated on a CPU thread independent of the GPU rendering framerate.

\section{A.5 Artist Feedback Prompts}

General:

- What first impression thoughts did you have when seeing the results or using this tool?

- What features were useful, and what was missing?

- What practical or technical aspects of this tool can help or hinder production usage of this technique?

Visual Results:

- How useful were the edit shapes the shading rig could generate? What shapes were missing or difficult to produce for shade editing?

- Did the edited shading look natural, or too geometric? 
- For what aesthetic purposes can the shading rig be useful? What aesthetic qualities were difficult to achieve?

Interaction:

- How difficult was it to control the results?

- Did the parameters behave as expected?

- How much did the shading shape parameters over-complicate the lighting workflow? 
Table A.1: Symbols and notation.

\begin{tabular}{|c|c|}
\hline Symbol & Definition \\
\hline$a$ & Anisotropy \\
\hline$s$ & Sharpness \\
\hline$w_{y}$ & Bend \\
\hline$w_{x}$ & Bulge \\
\hline$\theta_{r}$ & Rotation \\
\hline$\tau$ & Normal Smooth \\
\hline$d$ & Softness \\
\hline$G$ & Intensity Gain (for Intensity edits) \\
\hline$R$ & Radius of influence for a given edit \\
\hline $\mathbf{p}_{l}$ & Edit position / light position \\
\hline $\mathbf{p}_{o}$ & Light origin \\
\hline$(u, v)$ & Local texture space coordinates \\
\hline $\mathbf{p}_{w}$ & World position of shading point \\
\hline$I(x, y)$ & Intensity distribution function \\
\hline$\alpha$ & $\alpha:=1-a$ \\
\hline$\left(u_{w}, v_{w}\right)$ & Warped texture space coordinates \\
\hline $\mathbf{W}$ & $\mathbf{w}:=\left(w_{x}, w_{y}\right)^{\top}$ \\
\hline $\mathbf{u}$ & $\mathbf{u}:=(u, v)^{\top}$ \\
\hline$\theta_{w}(\mathbf{u})$ & Warping function \\
\hline$k_{w}$ & Warping coefficient for $\theta_{w}$ \\
\hline $\boldsymbol{R}(\theta)$ & 2D rotation matrix for angle $\theta$ \\
\hline$c(\theta)$ & Mask to remove repetitions from warping \\
\hline$f_{s}(x)$ & Distance fall-off function \\
\hline$k_{s}$ & Step-edge width for $f_{s}(x)$ \\
\hline$\omega$ & Attenuation factor based on distance fall-off \\
\hline $\mathbf{N}(\tau)$ & Smoothed normal vector of shading point \\
\hline $\mathbf{n}$ & Surface normal vector of shading point \\
\hline$\gamma$ & Attenuation factor based on $\mathbf{N}(\tau)$ \\
\hline $\mathbf{v}$ & Direction vector from $\mathbf{p}_{w}$ to $\mathbf{p}_{l}$ \\
\hline $\mathbf{p}_{c}$ & Centroid of surface mesh \\
\hline$d_{0}$ & Existing reflectance from conventional lighting \\
\hline$N_{I}$ & Number of Intensity Edits in the shading rig \\
\hline$T_{0}$ & Global toon shading threshold \\
\hline $\mathbf{B}_{1}$ & Lit region on surface \\
\hline $\mathbf{M}$ & Mask region of a given Mask edit \\
\hline$t_{m}$ & Threshold to produce the mask $\mathbf{M}$ \\
\hline$N_{M}$ & Number of Mask edits in the shading rig \\
\hline$\lambda$ & Spherical gaussian kernel width \\
\hline$\eta$ & Direction vector parameter to sample the spherical gaussian \\
\hline$\xi$ & Center axis of the spherical gaussian \\
\hline$\left[\mathbf{l}_{\mathrm{x}} \mathbf{l}_{\mathrm{y}} \mathbf{l}_{\mathrm{z}}\right]$ & Light space \\
\hline $\mathrm{v}_{\mathrm{l}}$ & Light space vector \\
\hline
\end{tabular}



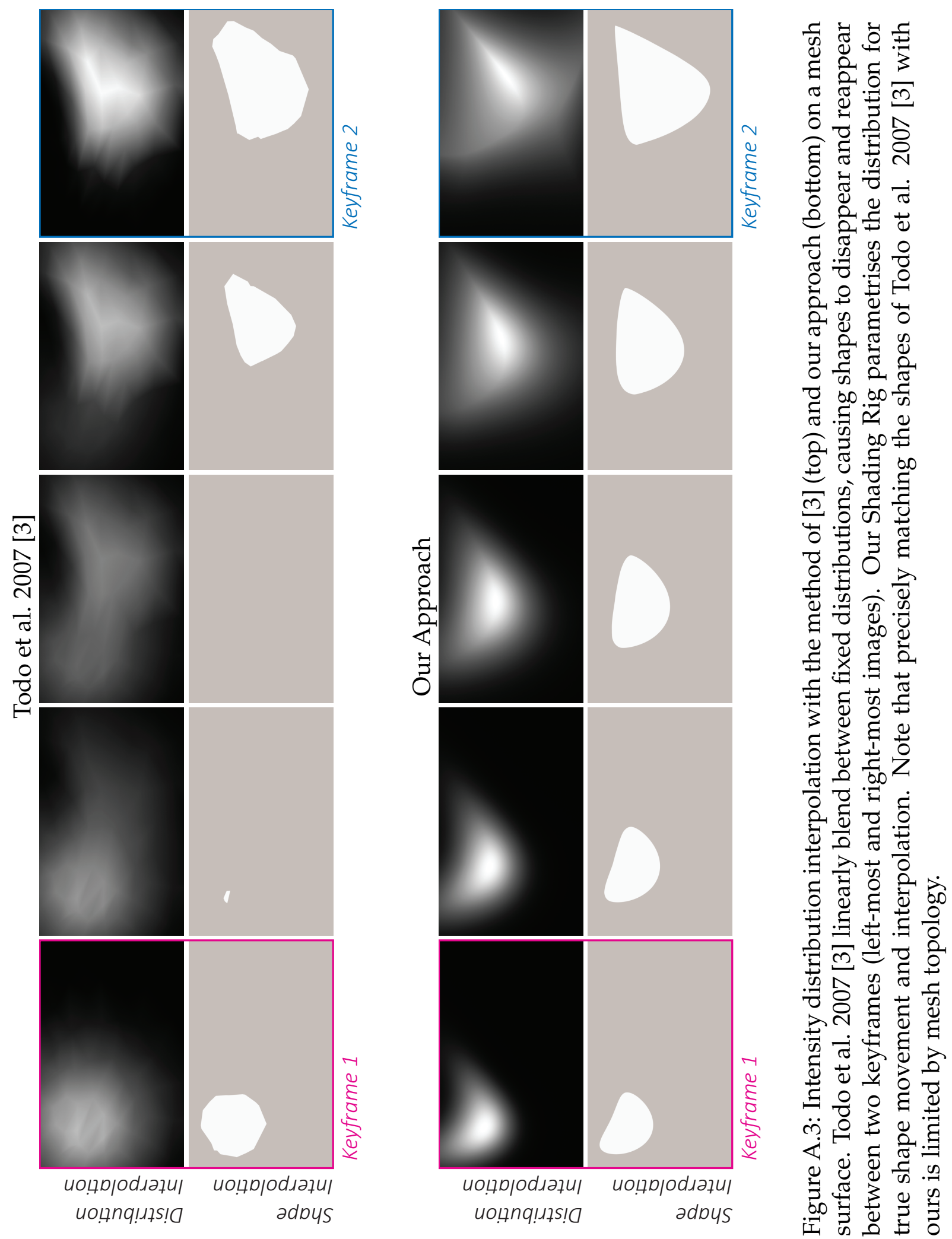


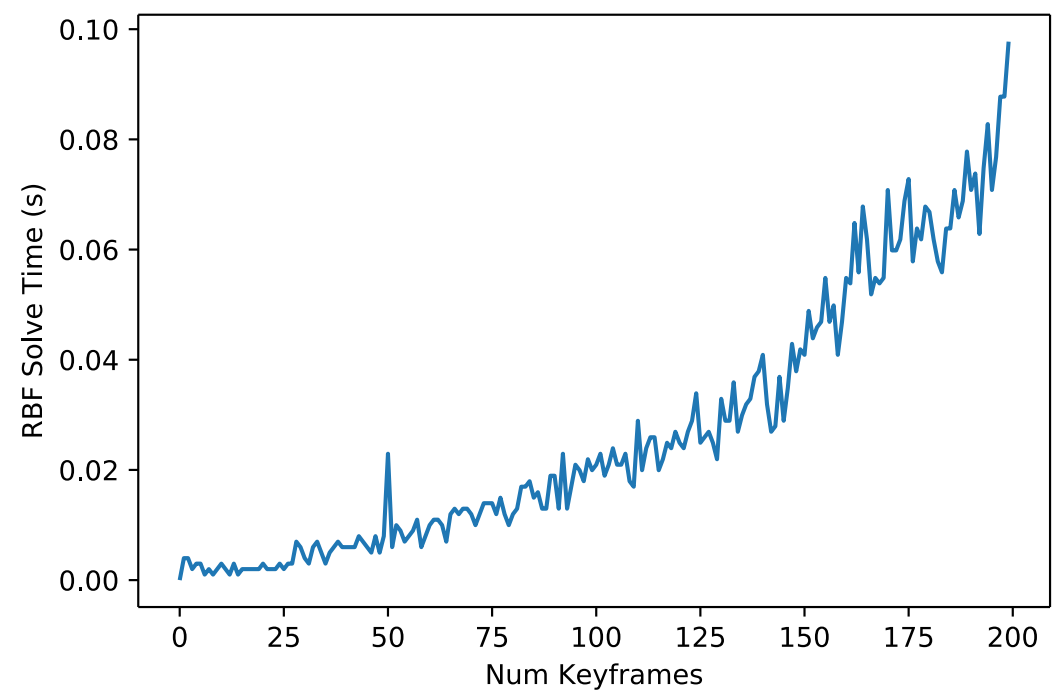

Figure A.4: Time taken to keyframe all parameters for one edit, as its number of keyframes increases, measured by the RBF solve time.

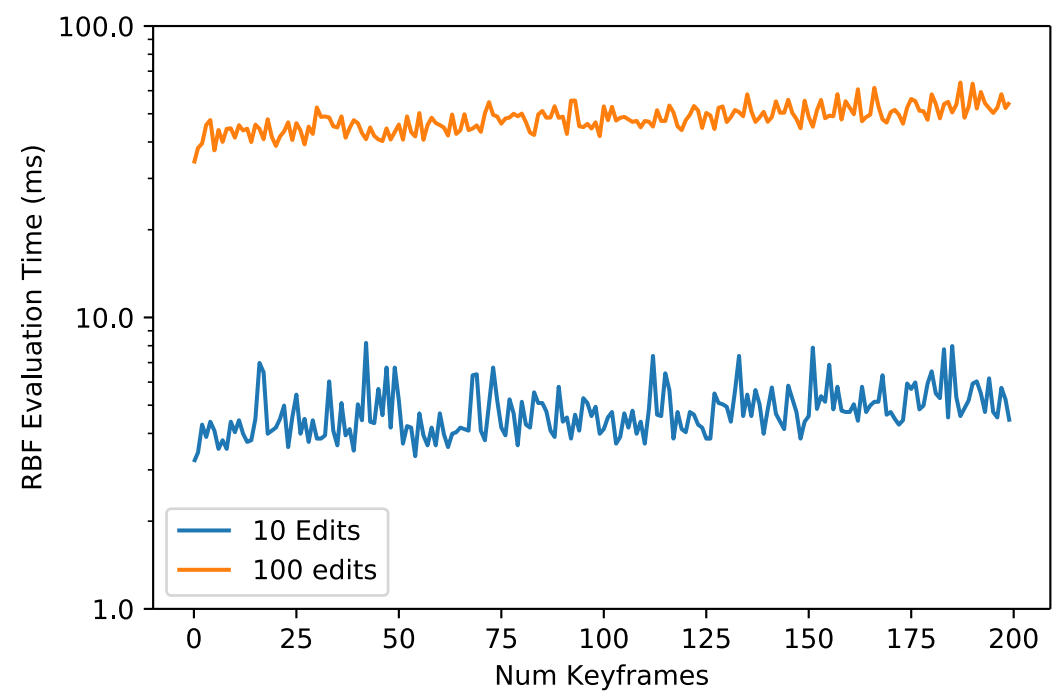

Figure A.5: RBF evaluation time (log scale) for shading rigs with 10 and 100 edits, as the number of keyframes per edit increases (all parameters keyframed). 


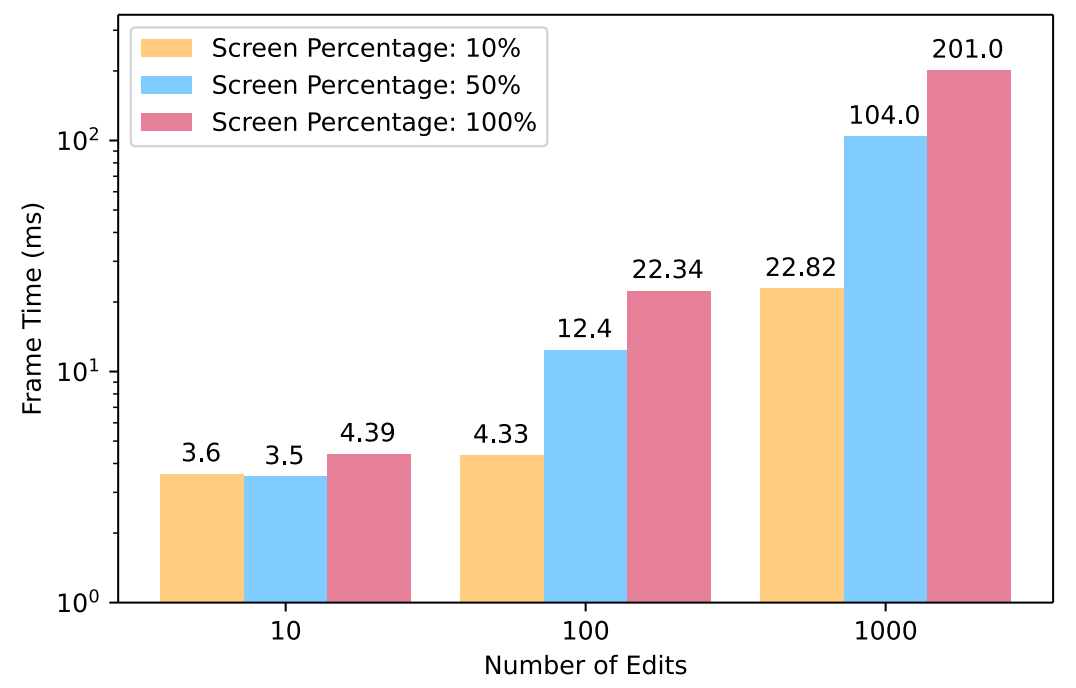

Figure A.6: GPU shading frame time (log scale) as the number of edits increases, at different screen percentages of $1920 \times 1080$ resolution. 


\section{Appendix}

\section{Ethics}

There were various user studies conducted in this thesis. Each user study involved image quality or experience evaluation. The following is the generic information sheet, generic consent form, and generic image quality questions used. The exact wording and setup is described in each chapter that has a user study. 


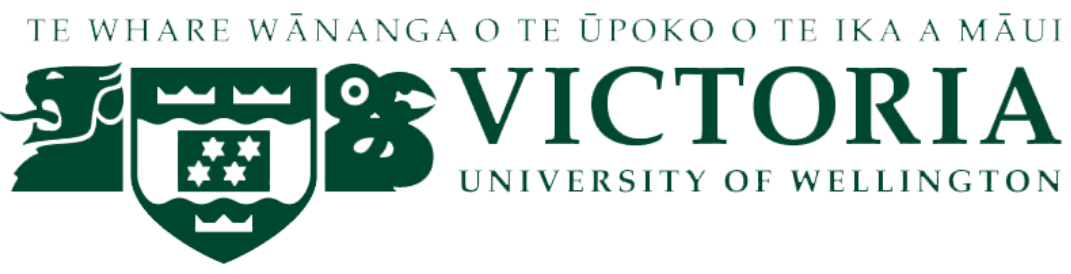

\section{Image Quality User Study Participant Consent Form}

Researcher: Lohit Petikam

School of Engineering and Computer Science, Victoria University of Wellington.

Please read the following notices. If you agree with them, please sign this form.

By choosing agree: I, the participant, understand and agree that:

- My participation in this research is voluntary and I am aware that I am able to withdraw within 1 week after my participation in the study.

- I am aware that, should I choose to withdraw, that the investigator may ask me why I have chosen to withdraw but I do not have to provide any reasons if I do not want to.

- I confirm that I have have been provided, read and understand the Participant Information Sheet.

- I have had the opportunity to ask any questions about this research and had them answered.

- I understand that all personal information will remain confidential and that all efforts will be made to ensure I cannot be identified.

- I agree that data gathered in this study may be stored anonymously and securely, and may be used for future research.

- I agree to take part in this study.

- If I have any further concerns and/or questions, I am aware I can use the following contacts:

- Student researcher: Lohit Petikam

email: lohit.petikam@gmail.com

- Supervisor: Taehyun Rhee

School of Engineering and Computer Science

phone: 044635233 x 7088 or email: taehyun.rhee@ecs.vuw.ac.nz.

- Human Ethics Committee Convener: Associate Professor Susan Corbett phone 044635480 or email susan.corbett@vuw.ac.nz. 


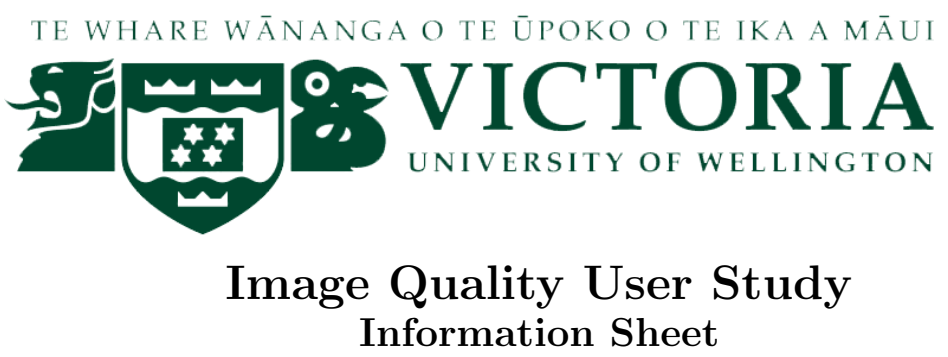

Researcher: Lohit Petikam

School of Engineering and Computer Science, Victoria University of Wellington.

You are invited to take part in a study investigating image quality. This study involves one session, during which you will view images and answer questions about them.

Data collected during this study will be stored in an anonymous form, in which no personally identifying information about you will be kept. You may withdraw from this study at any time.

This Participant Information Sheet will help you decide if youd like to take part. It sets out why I am doing the study, what your participation would involve, what the benefits and risks to you might be, and what would happen after the study ends. Feel free to ask the researcher any questions.

If you agree to take part in this study, you will be asked to accept a consent form.

Please make sure you have read and understood both this Information Sheet and the Consent Form before accepting.

This research has VUW Human Ethics Committee (HEC) approval.

What are the conditions for my participation in this study?

In order to participate in this study, you must:

- Be over 18 years of age

- Able to view images on a screen

- Not have any serious visual abnormalities (amblyopia, spherical aberrations, etc)

If you have a visual condition and are not sure if it will be an issue, please mention this to the researcher. Note that requiring prescription focal correction (i.e. long or short sightedness) is not an issue. If you are colour blind, you are still welcome to participate in the study, but you need to let the researcher know of your colour blind condition. 
What is the purpose of this study?

To determine how well our algorithm works in comparison with user evaluated data.

What will participating in this study involve?

You will be invited to view a series of images and answer questions for each one.

What are the possible benefits and/or risks of this study?

There are no risks with this study. The results of this study will benefit the researchers by providing a way of assessing the researcher's algorithms.

What happens once this study ends?

I will remove all identifying information about you from the results, and only store aggregated data: you may request from me these results, but they will not be tailored to you or mention you in any specific manner.

These aggregate results will be stored indefinitely.

The aggregates results of this study will used in my thesis, and in a proposed research paper to be published this year.

\section{If you have any questions, who can you contact?}

Student researcher: Lohit Petikam

email: lohit.petikam@gmail.com

Supervisor: Taehyun Rhee

School of Engineering and Computer Science

phone 044635233 x 7088or email taehyun.rhee@ecs.vuw.ac.nz

\section{Human Ethics Committee Information}

If you have any concerns about the ethical conduct of the research you may contact the Victoria University HEC Convener: Associate Professor Susan Corbett. Phone 044635480 or email susan.corbett@vuw.ac.nz.

Thank you very much for your time and help in making this study possible. 


\section{Bibliography}

[1] HDRIHaven, "HDRIHaven [Radiance Maps Database]." https: //hdrihaven.com/hdris/, 2020. Accessed: 29-06-2020.

[2] H. Todo, K. Anjyo, and S. Yokoyama, "Lit-sphere extension for artistic rendering," Vis. Comput., vol. 29, pp. 473-480, June 2013.

[3] H. Todo, K.-i. Anjyo, W. Baxter, and T. Igarashi, "Locally controllable stylized shading," ACM Trans. Graph., vol. 26, July 2007.

[4] G. Nader and G. Guennebaud, "Instant transport maps on 2d grids," $A C M$ Transactions on Graphics, vol. 37, no. 6, p. 13, 2018.

[5] B. Block, The visual story: Creating the visual structure of film, TV and digital media. CRC Press, 2013.

[6] M. Jutan and S. Ellis, "Director-centric virtual camera production tools for Rogue One," in ACM SIGGRAPH 2017 Talks, SIGGRAPH '17, (New York, NY, USA), Association for Computing Machinery, 2017.

[7] K. Margo, "Maturing the virtual production workflow: Interactive path tracing for filmmakers," in ACM SIGGRAPH 2014 Computer Animation Festival, SIGGRAPH '14, (New York, NY, USA), p. 1, Association for Computing Machinery, 2014.

[8] P. P. Srinivasan, B. Mildenhall, M. Tancik, J. T. Barron, R. Tucker, and N. Snavely, "Lighthouse: Predicting lighting volumes for spatially-coherent illumination," in CVPR, 2020. 
[9] P. A. Rauschnabel, A. Rossmann, and M. C. [tom Dieck], "An adoption framework for mobile augmented reality games: The case of Pokémon Go," Computers in Human Behavior, vol. 76, pp. 276 - 286, 2017.

[10] M. Leap, "Madefire and Magic Leap launch publishing into spacemadefire," Feb 2019.

[11] T. Rhee, A. Chalmers, M. Hicks, K. Kumagai, B. Allen, I. Loh, L. Petikam, and K. Anjyo, "MR360 interactive: playing with digital creatures in $360^{\circ}$ videos," in SIGGRAPH Asia 2018 Virtual \& Augmented Reality, pp. 1-2, 2018.

[12] P. Debevec, "Image-based lighting," in ACM SIGGRAPH 2006 Courses, pp. 4es, 2006.

[13] P. Debevec, "Rendering synthetic objects into real scenes: Bridging traditional and image-based graphics with global illumination and high dynamic range photography," in Proceedings of Computer Graphics and Interactive Techniques, SIGGRAPH '98, pp. 189-198, ACM, 1998.

[14] T. Rhee, L. Petikam, B. Allen, and A. Chalmers, "MR360: Mixed reality rendering for $360^{\circ}$ panoramic videos," IEEE Transactions on Visualization and Computer Graphics, vol. 23, no. 4, pp. 1379-1388, 2017.

[15] R. Brinkmann, The art and science of digital compositing: Techniques for visual effects, animation and motion graphics. Morgan Kaufmann, 2008.

[16] S. Moore and P. Wells, “Fundamentals of animation 2nd ed," 2016.

[17] S. Sint, Digital Portrait Photography: Art, Business and Style. Sterling Publishing Company, Inc., 2009.

[18] D. Landau, Lighting for cinematography: a practical guide to the art and craft of lighting for the moving image. A\&C Black, 2014.

[19] B. McLernon, Canon Speedlite System Digital Field Guide, 2nd Edition. John Wiley \& Sons, 2010.

[20] K. Malkiewicz and M. D. Mullen, Cinematography. Simon and Schuster, 2009. 
[21] W. Lidwell, K. Holden, and J. Butler, Universal principles of design, revised and updated: 125 ways to enhance usability, influence perception, increase appeal, make better design decisions, and teach through design. Rockport Pub, 2010.

[22] R. Napoli and C. Gloman, Scenic design and lighting techniques: A basic guide for theatre. Focal Press, 2013.

[23] T. O'Hailey, Hybrid animation: integrating 2D and 3D assets. Routledge, 2012.

[24] B. Tillman, Creative Character Design 2e. CRC Press, 2019.

[25] N. Boughen, LightWave 3D 8 Lighting. Wordware Publishing, Inc., 2004.

[26] J. Fišer, O. Jamriška, M. Lukáč, E. Shechtman, P. Asente, J. Lu, and D. Sỳkora, "StyLit: illumination-guided example-based stylization of $3 \mathrm{~d}$ renderings," ACM Transactions on Graphics (TOG), vol. 35, no. 4, p. 92, 2016.

[27] T.-W. Schmidt, F. Pellacini, D. Nowrouzezahrai, W. Jarosz, and C. Dachsbacher, "State of the art in artistic editing of appearance, lighting and material," in Computer Graphics Forum, vol. 35, pp. 216-233, Wiley Online Library, 2016.

[28] J. W. Hennessey, W. Li, B. Russell, E. Shechtman, and N. J. Mitra, “Transferring image-based edits for multi-channel compositing," ACM Transactions on Graphics (TOG), vol. 36, no. 6, pp. 1-16, 2017.

[29] A. Chalmers, J. J. Choi, and T. Rhee, "Perceptually optimised illumination for seamless composites," Pacific Graphics, The Eurographics Association, 2014.

[30] T. Iorns and T. Rhee, "Real-time image based lighting for 360-degree panoramic video," in Revised Selected Papers of the PSIVT 2015 Workshops on Image and Video Technology - LNCS, Volume 9555, pp. 139-151, 2016.

[31] B. Burley, D. Adler, M. J.-Y. Chiang, H. Driskill, R. Habel, P. Kelly, P. Kutz, Y. K. $\mathrm{Li}$, and D. Teece, "The design and evolution of Disney's hiperion renderer," ACM Transactions on Graphics, vol. 37, no. 3, p. 33, 2018. 
[32] L. Gruber, T. Langlotz, P. Sen, T. Höherer, and D. Schmalstieg, "Efficient and robust radiance transfer for probeless photorealistic augmented reality," in 2014 IEEE Virtual Reality (VR), pp. 15-20, IEEE, 2014.

[33] L. Gruber, J. Ventura, and D. Schmalstieg, "Image-space illumination for augmented reality in dynamic environments," in 2015 IEEE Virtual Reality (VR), pp. 127-134, March 2015.

[34] R. Koch, I. Schiller, B. Bartczak, F. Kellner, and K. Köser, “MixIn3D: 3d mixed reality with tof-camera.," in Dyn3D, pp. 126-141, Springer, 2009.

[35] K. Karsch, V. Hedau, D. Forsyth, and D. Hoiem, "Rendering synthetic objects into legacy photographs," in ACM Transactions on Graphics (TOG), vol. 30, p. 157, ACM, 2011.

[36] K. Karsch, K. Sunkavalli, S. Hadap, N. Carr, H. Jin, R. Fonte, M. Sittig, and D. Forsyth, "Automatic scene inference for $3 \mathrm{~d}$ object compositing," ACM Trans. Graph., vol. 33, pp. 32:1-32:15, June 2014.

[37] P. E. Debevec, C. J. Taylor, and J. Malik, "Modeling and rendering architecture from photographs: A hybrid geometry-and image-based approach," in Proceedings of the 23rd annual conference on Computer graphics and interactive techniques, pp. 11-20, ACM, 1996.

[38] T. Duff, "Deep compositing using lie algebras," ACM Transactions on Graphics (TOG), vol. 36, no. 3, p. 26, 2017.

[39] K. Jacobs, J.-D. Nahmias, C. Angus, A. Reche, C. Loscos, and A. Steed, "Automatic generation of consistent shadows for augmented reality," in Proceedings of Graphics Interface, pp. 113-120, Canadian Human-Computer Communications Society, 2005.

[40] I. P. Howard, Seeing in depth, Vol. 1: Basic mechanisms. University of Toronto Press, 2002.

[41] A. Dey, A. Cunningham, and C. Sandor, "Evaluating depth perception of photorealistic mixed reality visualizations for occluded objects in outdoor 
environments," in Proceedings of the 17th ACM Symposium on Virtual Reality Software and Technology, pp. 211-218, ACM, 2010.

[42] A. Dey, G. Jarvis, C. Sandor, and G. Reitmayr, “Tablet versus phone: Depth perception in handheld augmented reality," in Mixed and Augmented Reality (ISMAR), 2012 IEEE International Symposium on, pp. 187-196, IEEE, 2012.

[43] D. Dunn, C. Tippets, K. Torell, P. Kellnhofer, K. Akşit, P. Didyk, K. Myszkowski, D. Luebke, and H. Fuchs, "Wide field of view varifocal near-eye display using see-through deformable membrane mirrors," IEEE Transactions on Visualization and Computer Graphics, vol. 23, no. 4, pp. 13221331, 2017.

[44] J. Swan, M. A. Livingston, H. S. Smallman, D. Brown, Y. Baillot, J. L. Gabbard, and D. Hix, "A perceptual matching technique for depth judgments in optical, see-through augmented reality," in Virtual Reality Conference, 2006, pp. 19-26, IEEE, 2006.

[45] R. Messing and F. H. Durgin, "Distance perception and the visual horizon in head-mounted displays," ACM Transactions on Applied Perception (TAP), vol. 2, no. 3, pp. 234-250, 2005.

[46] K. Carnegie and T. Rhee, "Reducing visual discomfort with HMDs using dynamic depth of field," IEEE computer graphics and applications, vol. 35, no. 5, pp. 34-41, 2015.

[47] E. Langbehn, T. Raupp, G. Bruder, F. Steinicke, B. Bolte, and M. Lappe, "Visual blur in immersive virtual environments: does depth of field or motion blur affect distance and speed estimation?," in Proceedings of the 22nd ACM Conference on Virtual Reality Software and Technology, pp. 241-250, ACM, 2016.

[48] J. H. Clark, "Hierarchical geometric models for visible surface algorithms," Communications of the ACM, vol. 19, no. 10, pp. 547-554, 1976.

[49] D. P. Luebke, Level of detail for 3D graphics. Morgan Kaufmann, 2003.

[50] D. Luebke and B. Hallen, "Perceptually driven simplification for interactive rendering," in Rendering Techniques 2001, pp. 223-234, Springer, 2001. 
[51] M. Corsini, M.-C. Larabi, G. Lavoué, O. Petřík, L. Váša, and K. Wang, "Perceptual metrics for static and dynamic triangle meshes," in Computer Graphics Forum, vol. 32, pp. 101-125, Wiley Online Library, 2013.

[52] N. Sugano, H. Kato, and K. Tachibana, "The effects of shadow representation of virtual objects in augmented reality," in Mixed and Augmented Reality, 2003. Proceedings. The Second IEEE and ACM International Symposium on, pp. 76-83, IEEE, 2003.

[53] M. Weier, M. Stengel, T. Roth, P. Didyk, E. Eisemann, M. Eisemann, S. Grogorick, A. Hinkenjann, E. Kruijff, M. Magnor, et al., "Perception-driven accelerated rendering," in Computer Graphics Forum, vol. 36, pp. 611-643, Wiley Online Library, 2017.

[54] I. Yu, A. Cox, M. H. Kim, T. Ritschel, T. Grosch, C. Dachsbacher, and J. Kautz, "Perceptual influence of approximate visibility in indirect illumination," ACM Transactions on Applied Perception, vol. 6, no. 4, p. 24, 2009.

[55] M. Sattler, R. Sarlette, T. Mücken, and R. Klein, "Exploitation of human shadow perception for fast shadow rendering," in Proceedings of the 2nd symposium on Applied perception in graphics and visualization, pp. 131-134, ACM, 2005.

[56] G. Ramanarayanan, J. Ferwerda, B. Walter, and K. Bala, "Visual equivalence: towards a new standard for image fidelity," in ACM Transactions on Graphics (TOG), vol. 26, p. 76, ACM, 2007.

[57] S. J. Daly, "Visible differences predictor: an algorithm for the assessment of image fidelity," in Human Vision, Visual Processing, and Digital Display III, vol. 1666, pp. 2-16, International Society for Optics and Photonics, 1992.

[58] J. Křivánek, J. A. Ferwerda, and K. Bala, "Effects of global illumination approximations on material appearance," in ACM Transactions on Graphics (TOG), vol. 29, p. 112, ACM, 2010.

[59] G. Nakano, I. Kitahara, and Y. Ohta, "Generating perceptually-correct shadows for mixed reality," in Proceedings of the 7th IEEE/ACM International 
Symposium on Mixed and Augmented Reality, pp. 173-174, IEEE Computer Society, 2008.

[60] M. Borg, M. M. Paprocki, and C. B. Madsen, "Perceptual evaluation of photorealism in real-time 3d augmented reality," in Proc. of Computer Graphics Theory and Applications, 2014, pp. 1-10, IEEE, 2014.

[61] I. Daribo and B. Pesquet-Popescu, "Depth-aided image inpainting for novel view synthesis," in Multimedia Signal Processing (MMSP), 2010 IEEE International Workshop on, pp. 167-170, IEEE, 2010.

[62] R. Pajarola, M. Sainz, and Y. Meng, Depth-mesh objects: Fast depth-image meshing and warping. UCI-ICS Technical Report No. 03-02, Department of Information and Computer Science, University of California, Irvine, 2003.

[63] V. Hedau, D. Hoiem, and D. Forsyth, "Recovering the spatial layout of cluttered rooms," in Computer vision, 2009 IEEE 12th international conference on, pp. 1849-1856, IEEE, 2009.

[64] Y. Zhang, S. Song, P. Tan, and J. Xiao, "Panocontext: A whole-room 3d context model for panoramic scene understanding," in European Conference on Computer Vision, pp. 668-686, Springer, 2014.

[65] D. Eigen, C. Puhrsch, and R. Fergus, "Depth map prediction from a single image using a multi-scale deep network," in Advances in neural information processing systems, pp. 2366-2374, 2014.

[66] B. Peng, L. Zhang, and D. Zhang, "A survey of graph theoretical approaches to image segmentation," Pattern Recognition, vol. 46, no. 3, pp. 1020-1038, 2013.

[67] D. Hoiem, A. A. Efros, and M. Hebert, "Automatic photo pop-up," ACM transactions on graphics (TOG), vol. 24, no. 3, pp. 577-584, 2005.

[68] R. Guo, Q. Dai, and D. Hoiem, "Single-image shadow detection and removal using paired regions," in Computer Vision and Pattern Recognition (CVPR), 2011 IEEE Conference on, pp. 2033-2040, IEEE, 2011. 
[69] J.-F. Lalonde, A. A. Efros, and S. G. Narasimhan, “Detecting ground shadows in outdoor consumer photographs," in European conference on computer vision, pp. 322-335, Springer, Berlin, Heidelberg, 2010.

[70] D. H. Kelly, "Motion and vision. II. stabilized spatio-temporal threshold surface," Josa, vol. 69, no. 10, pp. 1340-1349, 1979.

[71] J. Huang, Z. Chen, D. Ceylan, and H. Jin, "6-DOF VR videos with a single 360-camera," in Virtual Reality, 2017 IEEE, pp. 37-44, IEEE, 2017.

[72] J. Xu, B. Stenger, T. Kerola, and T. Tung, "Pano2cad: Room layout from a single panorama image," in Applications of Computer Vision (WACV), 2017 IEEE Winter Conference on, pp. 354-362, IEEE, 2017.

[73] P. Hedman, S. Alsisan, R. Szeliski, and J. Kopf, "Casual 3D Photography," in ACM Transactions on Graphics (Proc. SIGGRAPH Asia), vol. 36, pp. 234:1-234:15, ACM, 2017.

[74] S. Zwerman and J. A. Okun, The VES Handbook of Visual Effects: Industry Standard VFX Practices and Procedures. CRC Press, 2014.

[75] J. Xiao, K. A. Ehinger, A. Oliva, and A. Torralba, "Recognizing scene viewpoint using panoramic place representation," in IEEE Conference on Computer Vision and Pattern Recognition, 2012.

[76] D. S. Lopes, R. K. dos Anjos, and J. A. Jorge, "Assessing the usability of tilebased interfaces to visually navigate 3-d parameter domains," International Journal of Human-Computer Studies, vol. 118, 2018.

[77] N. Elmqvist, P. Dragicevic, and J.-D. Fekete, "Rolling the dice: Multidimensional visual exploration using scatterplot matrix navigation," IEEE transactions on Visualization and Computer Graphics, vol. 14, no. 6, pp. 1539-1148, 2008.

[78] H. Sanftmann and D. Weiskopf, "3d scatterplot navigation," IEEE Transactions on Visualization and Computer Graphics, vol. 18, no. 11, pp. 1969-1978, 2012. 
[79] S. Theodoridis and K. Koutroumbas, Pattern Recognition, Fourth Edition. USA: Academic Press, Inc., 4th ed., 2008.

[80] R. Ramamoorthi and P. Hanrahan, "An efficient representation for irradiance environment maps," in Proceedings of the 28th annual conference on Computer graphics and interactive techniques, pp. 497-500, 2001.

[81] R. Ng, R. Ramamoorthi, and P. Hanrahan, "All-frequency shadows using non-linear wavelet lighting approximation," in ACM SIGGRAPH 2003 Papers, pp. 376-381, 2003.

[82] M.-A. Gardner, K. Sunkavalli, E. Yumer, X. Shen, E. Gambaretto, C. Gagné, and J.-F. Lalonde, "Learning to predict indoor illumination from a single image," ACM TOG, vol. 9, no. 4, 2017.

[83] T. Funkhouser, P. Min, M. Kazhdan, J. Chen, A. Halderman, D. Dobkin, and D. Jacobs, "A search engine for 3d models," ACM Transactions on Graphics (TOG), vol. 22, no. 1, pp. 83-105, 2003.

[84] M. Espadoto, R. M. Martins, A. Kerren, N. S. Hirata, and A. C. Telea, "Towards a quantitative survey of dimension reduction techniques," IEEE Transactions on Visualization and Computer Graphics, 2019.

[85] L. v. d. Maaten and G. Hinton, "Visualizing data using t-sne," Journal of machine learning research, vol. 9, no. Nov, pp. 2579-2605, 2008.

[86] I. Jolliffe, "Principal component analysis. briefings in bioinfomatics," New York: Springer, vol. 12, pp. 714-722, 2011.

[87] L. McInnes, J. Healy, and J. Melville, "Umap: Uniform manifold approximation and projection for dimension reduction," arXiv preprint arXiv:1802.03426, 2018.

[88] W. Baxter and K. Anjyo, "Latent Doodle Space," Computer Graphics Forum, 2006.

[89] J. Marks, B. Andalman, P. A. Beardsley, W. Freeman, S. Gibson, J. Hodgins, T. Kang, B. Mirtich, H. Pfister, W. Ruml, et al., “Design galleries: A general 
approach to setting parameters for computer graphics and animation," in Proceedings of the 24th annual conference on Computer graphics and interactive techniques, pp. 389-400, 1997.

[90] P. Hermosilla, S. Maisch, T. Ritschel, and T. Ropinski, "Deep-learning the latent space of light transport," in Computer Graphics Forum, vol. 38, pp. 207217, 2019.

[91] A. Chalmers, J. Lewis, P. Hillman, C. Tait, and T. Rhee, "Sky Browser: Search for HDR sky maps," 2014.

[92] J. Zhang and J.-F. Lalonde, "Learning high dynamic range from outdoor panoramas," in IEEE International Conference on Computer Vision, 2017.

[93] Y. Hold-Geoffroy, A. Athawale, and J.-F. Lalonde, "Deep sky modeling for single image outdoor lighting estimation," in Proceedings of the IEEE Conference on Computer Vision and Pattern Recognition, pp. 6927-6935, 2019.

[94] P. Barla, J. Thollot, and L. Markosian, "X-toon: An extended toon shader," in Proceedings of the 4th International Symposium on Non-Photorealistic Animation and Rendering, (New York, NY, USA), Association for Computing Machinery, 2006.

[95] DutchSkies360, “Dutch Skies 360 [Radiance Maps Database]." http: / /www . dutch 360 hdr.com/, 2017. Accessed: 05-05-2015.

[96] HDRIHub, "HDRI Hub [Radiance Maps Database]." http://www.hdrihub.com/, 2017. Accessed: 26-03-2015.

[97] HDRMaps, "HDR Maps [Radiance Maps Database]." http://hdrmaps . com/, 2017. Accessed: 25-05-2015.

[98] R. Ramamoorthi and P. Hanrahan, "Frequency space environment map rendering," in ACM TOG, vol. 21, 2002.

[99] C. I. de l'Eclairage, "Recommendations on uniform color spaces, colordifference equations, psychometric color terms," Paris: CIE, 1978. 
[100] D. N. Wood, A. Finkelstein, J. F. Hughes, C. E. Thayer, and D. H. Salesin, "Multiperspective panoramas for cel animation," in Proceedings of the 24th annual conference on Computer graphics and interactive techniques, pp. 243-250, ACM Press/Addison-Wesley Publishing Co., 1997.

[101] Y. Katsura and K. Anjyo, "Anime perspective," in ACM SIGGRAPH 2007 sketches, p. 75, ACM, 2007.

[102] J. C. Motomura, "GuiltyGearXrd's art style : The $x$ factor between $2 \mathrm{~d}$ and 3d," Mar 2015.

[103] J. Guertault, E. Setbon, and P. Martishevsky, "Manga stylized rendering in VR," in SIGGRAPH Asia 2018 Courses, SA '18, (New York, NY, USA), pp. 13:113:61, ACM, 2018.

[104] A. Lake, C. Marshall, M. Harris, and M. Blackstein, "Stylized rendering techniques for scalable real-time $3 \mathrm{~d}$ animation," in Proceedings of the 1st international symposium on Non-photorealistic animation and rendering, pp. 1320, ACM, 2000.

[105] O. Jamriška, Š. Sochorová, O. Texler, M. Lukáč, J. Fišer, J. Lu, E. Shechtman, and D. Sỳkora, "Stylizing video by example," ACM Transactions on Graphics (TOG), vol. 38, no. 4, p. 107, 2019.

[106] M. Dokter, J. Hladky, M. Parger, D. Schmalstieg, H.-P. Seidel, and M. Steinberger, "Hierarchical rasterization of curved primitives for vector graphics rendering on the GPU," Computer Graphics Forum, vol. 38, no. 2, pp. 93-103, 2019.

[107] G. Turk and J. F. O'Brien, "Shape transformation using variational implicit functions," in ACM SIGGRAPH 2005 Courses, SIGGRAPH '05, (New York, NY, USA), ACM, 2005.

[108] P. Barla, J. Thollot, and L. Markosian, "X-toon: an extended toon shader," in Proceedings of the 4th international symposium on Non-photorealistic animation and rendering, pp. 127-132, ACM, 2006. 
[109] M. Hudon, M. Grogan, R. Pagés, J. Ondřej, and A. Smolić, "2DToonShade: A stroke based toon shading system," Computers E Graphics: X, vol. 1, p. 100003, 2019.

[110] R. Pacanowski, X. Granier, C. Schlick, and P. Poulin, "Sketch and paintbased interface for highlight modeling," in Proceedings of the Fifth Eurographics Conference on Sketch-Based Interfaces and Modeling, SBM'08, (Goslar, DEU), p. 17-23, Eurographics Association, 2008.

[111] D. Vanderhaeghe, R. Vergne, P. Barla, and W. Baxter, "Dynamic Stylized Shading Primitives," in NPAR '11:Proceedings of the 8th International Symposium on Non-Photorealistic Animation and Rendering, (Vancouver, Canada), pp. 99104, Aug. 2011. Honorable Mention in Rendering.

[112] P. Bénard, A. Hertzmann, and M. Kass, "Computing smooth surface contours with accurate topology," ACM Trans. Graph., vol. 33, no. 2, 2014.

[113] M. Arief, K. Kondo, K. Mikami, H. Todo, and Y. Yamaguchi, "Controllable region via texture projection for stylized shading," in Proceedings of the 14th ACM SIGGRAPH International Conference on Virtual Reality Continuum and Its Applications in Industry, VRCAI '15, (New York, NY, USA), pp. 35-38, ACM, 2015.

[114] K.-i. Anjyo and K. Hiramitsu, "Stylized highlights for cartoon rendering and animation," IEEE Computer Graphics and Applications, vol. 23, no. 4, pp. 54-61, 2003.

[115] M. Okabe, Y. Matsushita, L. Shen, and T. Igarashi, "Illumination brush: Interactive design of all-frequency lighting," in 15th Pacific Conference on Computer Graphics and Applications (PG'07), pp. 171-180, Oct 2007.

[116] M. Tada, Y. Dobashi, and T. Yamamoto, "Feature-based interpolation for the interactive editing of shading effects," in Proceedings of the 11th ACM SIGGRAPH International Conference on Virtual-Reality Continuum and its Applications in Industry, pp. 47-50, 2012. 
[117] R. D. Kalnins, L. Markosian, B. J. Meier, M. A. Kowalski, J. C. Lee, P. L. Davidson, M. Webb, J. F. Hughes, and A. Finkelstein, "WYSIWYG NPR: Drawing strokes directly on 3D models," ACM Transactions on Graphics (Proc. SIGGRAPH), vol. 21, pp. 755-762, July 2002.

[118] O. Mattausch, T. Igarashi, and M. Wimmer, "Freeform shadow boundary editing," Computer Graphics Forum, vol. 32, no. 2pt2, pp. 175-184, 2013.

[119] F. Pellacini, P. Tole, and D. P. Greenberg, "A user interface for interactive cinematic shadow design," ACM Transactions on Graphics (TOG), vol. 21, no. 3, pp. 563-566, 2002.

[120] T. Ritschel, T. Thormählen, C. Dachsbacher, J. Kautz, and H.-P. Seidel, "Interactive on-surface signal deformation," ACM Trans. Graph., vol. 29, July 2010.

[121] A. Hertzmann, C. E. Jacobs, N. Oliver, B. Curless, and D. H. Salesin, "Image analogies," in Proceedings of the 28th annual conference on Computer graphics and interactive techniques, pp. 327-340, ACM, 2001.

[122] P. Bénard, F. Cole, M. Kass, I. Mordatch, J. Hegarty, M. S. Senn, K. Fleischer, D. Pesare, and K. Breeden, "Stylizing animation by example," $A C M$ Transactions on Graphics (TOG), vol. 32, no. 4, p. 119, 2013.

[123] D. Sýkora, O. Jamriška, O. Texler, J. Fišer, M. Lukáč, J. Lu, and E. Shechtman, "StyleBlit: Fast example-based stylization with local guidance," Computer Graphics Forum, vol. 38, no. 2, pp. 83-91, 2019.

[124] D. Seyb, A. Jacobson, D. Nowrouzezahrai, and W. Jarosz, "Non-linear sphere tracing for rendering deformed signed distance fields," ACM Trans. Graph., vol. 38, pp. 229:1-229:12, Nov. 2019.

[125] C. Troftgruben, Learning Anime Studio. Packt Publishing, 2014.

[126] H. Lieng, F. P. Tasse, J. Kosinka, and N. A. Dodgson, "Shading curves: Vectorbased drawing with explicit gradient control," Computer Graphics Forum, 2015. 
[127] E. Eisemann, H. Winnemöller, J. C. Hart, and D. Salesin, "Stylized vector art from 3d models with region support," in Computer Graphics Forum, vol. 27, pp. 1199-1207, Wiley Online Library, 2008.

[128] C. Loop and J. Blinn, "Resolution independent curve rendering using programmable graphics hardware," in ACM Transactions on Graphics (TOG), vol. 24, pp. 1000-1009, ACM, 2005.

[129] M. J. Kilgard and J. Bolz, "GPU-accelerated path rendering," ACM Transactions on Graphics (TOG), vol. 31, no. 6, pp. 1-10, 2012.

[130] N. Ray, X. Cavin, and B. Lévy, "Vector texture maps on the GPU," Inst. ALICE (Algorithms, Comput., Geometry Image Dept. INRIA Nancy Grand-Est/Loria), Tech. Rep. ALICE-TR-05-003, 2005.

[131] L. Wang, K. Zhou, Y. Yu, and B. Guo, "Vector solid textures," in ACM Transactions on Graphics (TOG), vol. 29, p. 86, ACM, 2010.

[132] X. Sun, G. Xie, Y. Dong, S. Lin, W. Xu, W. Wang, X. Tong, and B. Guo, "Diffusion curve textures for resolution independent texture mapping.," ACM Trans. Graph., vol. 31, no. 4, pp. 74-1, 2012.

[133] N. Bonneel, M. Van De Panne, S. Paris, and W. Heidrich, "Displacement interpolation using lagrangian mass transport," in ACM Transactions on Graphics (TOG), vol. 30, p. 158, ACM, 2011.

[134] J. Solomon, F. De Goes, G. Peyré, M. Cuturi, A. Butscher, A. Nguyen, T. Du, and L. Guibas, "Convolutional wasserstein distances: Efficient optimal transportation on geometric domains," ACM Transactions on Graphics (TOG), vol. 34, no. 4, p. 66, 2015.

[135] Z. Shu, S. Hadap, E. Shechtman, K. Sunkavalli, S. Paris, and D. Samaras, "Portrait lighting transfer using a mass transport approach," ACM Transactions on Graphics (TOG), vol. 37, no. 1, p. 2, 2018.

[136] J. Birn, Digital lighting E rendering. New Riders Press, 2000. 
[137] E. Keller and M. Dayan, Maya Visual Effects The Innovator's Guide: Autodesk Official Press. Sybex, 2013.

[138] B. Hogarth, Dynamic Light and Shade. ERIC, 1991.

[139] S. McCloud, Understanding comics: The invisible art. 1993.

[140] Y.-T. Tsai and Z.-C. Shih, "All-frequency precomputed radiance transfer using spherical radial basis functions and clustered tensor approximation," ACM Trans. Graph., vol. 25, p. 967-976, July 2006.

[141] P. Wright, Cinematic Portraits: How to Create Classic Hollywood Photography. Amherst Media, 2015.

[142] P.-P. J. Sloan, W. Martin, A. Gooch, and B. Gooch, “The lit sphere: A model for capturing NPR shading from art," in Graphics Interface, vol. 2001, pp. 143-150, 2001.

[143] G. Peyré, M. Cuturi, et al., "Computational optimal transport: With applications to data science," Foundations and Trends ${ }^{\circledR}$ in Machine Learning, vol. 11, no. 5-6, pp. 355-607, 2019.

[144] W. B. Kerr and F. Pellacini, "Toward evaluating lighting design interface paradigms for novice users," ACM Trans. Graph., vol. 28, July 2009.

[145] F. Luan, S. Paris, E. Shechtman, and K. Bala, "Deep painterly harmonization," in Computer Graphics Forum, vol. 37, pp. 95-106, Wiley Online Library, 2018.

[146] A. Bousseau, J. P. O'shea, F. Durand, R. Ramamoorthi, and M. Agrawala, "Gloss perception in painterly and cartoon rendering," ACM Transactions on Graphics (TOG), vol. 32, no. 2, pp. 1-13, 2013.

[147] R. Schmidt, C. Grimm, and B. Wyvill, "Interactive decal compositing with discrete exponential maps," ACM Trans. Graph., vol. 25, p. 605-613, July 2006. 
